\title{
Functional gene analysis in cultured vertebrate cells using siRNA mediated gene silencing
}

\author{
PhD Thesis \\ in partial fulfilment of the requirements \\ for the degree "Doctor of Philosophy (PhD)" \\ in the Molecular Biology Program \\ at the Georg August University Göttingen, \\ Faculty of Biology
}

\author{
submitted by \\ Jens Gruber
}

born in

Northeim

2004 
The experimental part of this thesis was performed at the

\section{Max-Planck-Institute for biophysical Chemistry Department of Biochemistry and Cell Biology Göttingen, Germany}

$1^{\text {st }}$ Referee: Prof. Dr. Mary Osborn

$2^{\text {nd }}$ Referee: Prof. Dr. Ralf Ficner

Date of submission of the PhD Thesis: $\quad$ December $\mathbf{2 3}^{\text {rd }}, 2004$

Date of thesis defense: $\quad$ February $\mathbf{1 0}^{\text {th }}, \mathbf{2 0 0 5}$ 
for my parents

and eva 


\begin{tabular}{|c|c|c|}
\hline Chapter & Title & Page \\
\hline & Abbreviations & \\
\hline I. & INTRODUCTION & 1 \\
\hline I.1 & RNA interference & 1 \\
\hline I.2 & The human endoprotease FACE1 & 6 \\
\hline I.3 & Miosis related targets for RNAi & 10 \\
\hline 1.3 .1 & Eukaryotic cell division & 10 \\
\hline I.3.2 & The human coiled coil protein astrin & 12 \\
\hline I.4 & Rational design of functional siRNAs & 13 \\
\hline I.5 & siRNA delivery by osmotic lysis of pinosomes & 15 \\
\hline I.6 & RNA interference in the zebrafish (Danio rerio) & 16 \\
\hline II. & MATERIALS AND METHODS & 20 \\
\hline II.1 & Materials & 20 \\
\hline II.1.1 & Chemicals and Enzymes & 20 \\
\hline II.1.2 & siRNA and nucleotides & 20 \\
\hline II.1.3 & Machines & 20 \\
\hline II.1.4 & Buffers and Solutions & 21 \\
\hline II.1.5 & Media and Culture Plates & 21 \\
\hline II.1.6 & Bacterial Host Strains & 22 \\
\hline II.1.7 & Vectors & 22 \\
\hline II.1.8 & Cell lines & 23 \\
\hline II.1.9 & Software & 23 \\
\hline II.2 & Molecular biology methods & 24 \\
\hline II.2.1 & $\begin{array}{l}\text { Purification of E. coli plasmids for analysis } \\
\text { (Miniprep) }\end{array}$ & 24 \\
\hline II.2.1.1 & Preparative Plasmid Purification (Midiprep) & 25 \\
\hline II.2.2 & Agarose Gel Electrophoresis & 25 \\
\hline II.2.3 & DNA-elution from agarose gels & 25 \\
\hline II.2.4 & Polymerase Chain Reaction & 26 \\
\hline
\end{tabular}




\begin{tabular}{|c|c|c|}
\hline II.2.4.1 & Primer design & 26 \\
\hline II.2.4.2 & Mutagenesis PCR for astrin amplification & 27 \\
\hline II. 2.5 & Restriction digestion of DNA & 28 \\
\hline II. 2.6 & Ligation reactions & 28 \\
\hline II. 2.7 & Transformation of competent bacteria & 29 \\
\hline II. 2.8 & Determination of nucleic acid concentration & 30 \\
\hline II.3 & Protein biochemical methods & 30 \\
\hline II.3.1 & Discontinuous polyacrylamide gel electrophoresis & 30 \\
\hline II.3.1.1 & Coomassie staining & 31 \\
\hline II.3.2 & Protein Expression & 32 \\
\hline II.3.3 & Purification of overexpressed proteins & 32 \\
\hline II.3.4 & Purification of polyclonal antibodies & 34 \\
\hline II.4 & siRNA techniques & 35 \\
\hline II.4.1 & $\begin{array}{l}\text { Selection of siRNA sequences for targeting specific } \\
\text { mRNAs }\end{array}$ & 35 \\
\hline II.4.2 & Preparation of siRNA Duplexes & 38 \\
\hline II.4.3 & $\begin{array}{l}\text { Transient transfection of human cell lines } \\
\text { with siRNA duplexes }\end{array}$ & 38 \\
\hline II.4.3.1 & Preparation of cells & 39 \\
\hline II.4.3.2 & Transfection of siRNA duplexes & 39 \\
\hline II.4.3.3 & Transfection with cationic liposomes & 39 \\
\hline II.4.3.4 & Microinjection of siRNAs & 40 \\
\hline II.4.3.5 & Pinocytosis mediated transfection of siRNAs & 41 \\
\hline II.4.3.6 & Transfection by electroporation & 42 \\
\hline II.4.4 & Detection of siRNA mediated gene silencing & 42 \\
\hline II.4.4.1 & Phase contrast microscopy & 43 \\
\hline II.4.4.2 & Indirect immunofluorescence microscopy & 43 \\
\hline II.4.4.3 & Microscopic growth rate determination (CellScreen) & 44 \\
\hline II.4.4.4 & Immunoblotting & 45 \\
\hline II.4.4.5 & TUNEL test for apoptosis detection & 47 \\
\hline II.4.4.6 & $\begin{array}{l}\text { Flow cytometry measurements to detect cell cycle } \\
\text { Distribution, cellular DNA contents and apoptosis }\end{array}$ & 48 \\
\hline II.4.4.7 & Determination of total cell numbers using the Cell & 49 \\
\hline
\end{tabular}


Counter and Analysis System (CASY ${ }^{\circledR}$ )

II.4.4.8 The branched DNA assay mRNA quantitation

III. RESULTS 52

III.1. Human Targets I: RNAi against the human 52 endoprotease FACE1

III.1.1 The endoprotease FACE1 is required for the 52 processing of prelamin A in HeLa cells

III.1.2 FACE1 knockdown causes changes in nuclear $\quad 54$ morphology

III.1.3 FACE1 knockdown caused aberrant mitosis and $\quad 55$ apoptosis

III.1.4 The phenotypic changes after FACE1 silencing are 57 due to the increase in prelamin A

III.1.5 Location of FACE1 in HeLa cells 60

III.1.6 Overview of results from RNAi against FACE1 61

III.2.1 Human targets 2; the mitotic spindle associated 63 protein astrin and mitosis related proteins

III.2.1.1 Astrin is an essential protein of the spindle 63

III.2.1.2 Cells display disturbed spindle allignment afetr astrin 64 silencing

III.2.1.3 Two additional siRNAs against astrin did not 66 efficiently knockdown

$\begin{array}{lll}\text { III.2.2 Silencing of mitosis related proteins } & 67\end{array}$

III.2.2.1 Silencing of kinetochore proteins and the cohesion $\quad 69$ complex

III.2.2.2 Silencing of kinesin related proteins $\quad 72$

$\begin{array}{lll}\text { III.2.2.3 Silencing of stathmin and katanin } & 74\end{array}$

III.2.3 Overview of results from RNAi against astrin and 75 mitosis related proteins

III.3 Anatomy of siRNAs 76

III.3.1 Silencing of lamin A/C with self complementary 76 asRNAs 


\begin{tabular}{|c|c|c|}
\hline III.3.2 & Two target site siRNAs & 82 \\
\hline III.3.3 & Dual targeting siRNAs & 85 \\
\hline III.3.4 & SiRNA rescue & 88 \\
\hline III.3.5 & Results overview & 89 \\
\hline III.4. & $\begin{array}{l}\text { Transfection of siRNAs by osmotic lysis of loaded } \\
\text { pinosomes }\end{array}$ & 91 \\
\hline III.4.1 & Strategy and background & 91 \\
\hline III.4.2 & $\begin{array}{l}\text { Delivery of siRNAs to cultured mammalian cells by } \\
\text { osmotic lysis of pinosomes }\end{array}$ & 91 \\
\hline III.4.3 & Released siRNAs induce RNAi & 93 \\
\hline III.4.4 & $\begin{array}{l}\text { Repeating the osmotic lysis of pinosomes procedure } \\
\text { increases the silencing efficiency to that obtained with } \\
\text { cationic liposomes }\end{array}$ & 94 \\
\hline III.4.5 & $\begin{array}{l}\text { Increased loading times and higher siRNA } \\
\text { concentrations increase silencing efficiencies }\end{array}$ & 96 \\
\hline III.4.6 & $\begin{array}{l}\text { Duration of transient knockdown is comparable after } \\
\text { pinocytosis and liposome based siRNA delivery }\end{array}$ & 97 \\
\hline III.4.7 & $\begin{array}{l}\text { Pinocytosis mediated transfection is a useful method } \\
\text { for siRNA silencing of different targets }\end{array}$ & 99 \\
\hline III.4.8 & $\begin{array}{l}\text { Overview of results from pinocytosis mediated siRNA } \\
\text { delivery }\end{array}$ & 101 \\
\hline III.5 & RNA interference in the zebrafish (Danio rerio) & 102 \\
\hline III.5.1 & $\begin{array}{l}\text { Generation of specific siRNAs targeting exogenous } \\
\text { and endogenous mRNAs }\end{array}$ & 102 \\
\hline III.5.2 & $\begin{array}{l}\text { Silencing of exogenous mGFP in cultured zebrafish } \\
\text { cells }\end{array}$ & 102 \\
\hline III.5.3 & $\begin{array}{l}\text { Silencing of endogenous genes in cultured zebrafish } \\
\text { cells of adult and embryonic origin }\end{array}$ & 104 \\
\hline III.5.4 & $\begin{array}{l}\text { Overview of results obtained with RNAi in Danio } \\
\text { rerio }\end{array}$ & 108 \\
\hline IV. & DISCUSSION & 109 \\
\hline IV.1 & RNAi against FACE1 & 109 \\
\hline
\end{tabular}




\begin{tabular}{|c|c|c|}
\hline IV.1.1 & FACE1 was successfully silenced & 109 \\
\hline IV.1.2 & Accumulation of prelamin A & 109 \\
\hline IV.1.3 & $\begin{array}{l}\text { FACE1 knockdown phenotypes and parallels to } \\
\text { progeria }\end{array}$ & 110 \\
\hline IV.1.4 & Conclusions after FACE1 knockdown & 113 \\
\hline IV.2. & Astrin and mitosis related proteins & 113 \\
\hline IV.3. & Anatomy of siRNAs & 116 \\
\hline IV.3.1 & Palindromic siRNAs & 116 \\
\hline IV.3.1 & $\begin{array}{l}\text { as/as duplexes and dual targeting of lamin } \mathrm{A} / \mathrm{C} \text { and } \\
\text { emerin }\end{array}$ & 117 \\
\hline IV.3.1 & Rescue of siRNAs by sense strand modifications & 118 \\
\hline IV.4 & Pinocytosis mediated siRNA delivery & 122 \\
\hline IV.5 & RNAi in Danio rerio & 123 \\
\hline IV.5.1 & $\begin{array}{l}\text { Delivery of siRNAs into cultured zebrafish cells } \\
\text { induces specific RNAi }\end{array}$ & 123 \\
\hline IV.6 & Conclusions & 125 \\
\hline V. & ACKNOWLEDGEMENTS & 127 \\
\hline VI. & REFERENCES & 128 \\
\hline VII. & SUMMARY & 144 \\
\hline \multirow[t]{5}{*}{ VIII. } & APPENDIX & 146 \\
\hline & List of tables and figures & 147 \\
\hline & Statement of the originality of the data & 148 \\
\hline & Publications & 149 \\
\hline & Curriculum vitae & 150 \\
\hline
\end{tabular}




\begin{tabular}{|c|c|}
\hline & Abbreviations \\
\hline aa & amino acid \\
\hline $\mathrm{ab}$ & antibody \\
\hline APS & ammoniumperoxodisulfate \\
\hline Amp & Ampicillin \\
\hline ATP & adenosine triphosphate \\
\hline ß-ME & beta mercaptoethanol \\
\hline BSA & bovine serum albumin \\
\hline$b p$ & base pair \\
\hline C- & carboxy- \\
\hline cDNA & complementary DNA \\
\hline $\mathrm{Da}$ & Dalton \\
\hline DAPI & $4^{\prime}-6^{\prime}$-diamidin-2'-phenylindol-dihydrochlorid \\
\hline DMEM & Dulbecco's modified Eagle's medium \\
\hline DMSO & dimethysulfoxide \\
\hline DNA & deoxyribonucleic acid \\
\hline dNTP & desoxynucleotide triphosphate \\
\hline E. coli & Escherichia coli \\
\hline ECL & enhanced chemiluminescence \\
\hline EDTA & ethylendiamine tetraacetate \\
\hline EMBL & European Molecular Biology Laboratory \\
\hline EST & expressed sequence tag \\
\hline $\mathrm{Gu}-\mathrm{HCL}$ & guanidium hydrochloride \\
\hline FPLC & fast performance liquid chromatography \\
\hline h & hour(s) \\
\hline HGPS & Hutchinson Gilford Progeria Syndrome \\
\hline HPLC & high pressure liquid chromatography \\
\hline HRP & horse radish peroxidase \\
\hline $\operatorname{IgG}$ & immunglobulin $\mathrm{G}$ \\
\hline IPTG & isopropyl-ß-D-thiogalactopyranosid \\
\hline $\mathrm{k}$ & kilo \\
\hline & kilo Dalton \\
\hline
\end{tabular}




\begin{tabular}{|c|c|}
\hline MAP & microtubular associated protein \\
\hline MCS & multiple cloning site \\
\hline $\min$ & minute(s) \\
\hline $\mathrm{m}$ & milli \\
\hline$\mu$ & micro \\
\hline MTOC & microtubular organising centre \\
\hline mRNA & messenger RNA \\
\hline MW & relative molecular weight \\
\hline $\mathrm{N}$ & amino- \\
\hline $\mathrm{nt}$ & nucleotide \\
\hline OD & optical density \\
\hline ORF & open reading frame \\
\hline PAGE & polyacrylamide gel electrophoresis \\
\hline PBS & phosphate buffered saline \\
\hline PCR & polymerase chain reaction \\
\hline PTGS & post-transcriptional gene silencing \\
\hline RNA & ribonucleic acid \\
\hline ss & single stranded \\
\hline ds & double stranded \\
\hline $\mathrm{s}$ & sense \\
\hline as & antisense \\
\hline rpm & revolutions per minute \\
\hline $\mathrm{s}$ & second(s) \\
\hline SDS & sodium dodecyl sulphate \\
\hline siRNA & small interfering RNA \\
\hline shRNA & short hairpin RNA \\
\hline TBS & Tris(-HCl) buffered saline \\
\hline Tris & Tris(hydroxymethyl)-aminomethane \\
\hline $\mathrm{U}$ & Unit \\
\hline wt & wild type \\
\hline $\mathrm{xg}$ & $\mathrm{x}$-fold gravity \\
\hline
\end{tabular}

amino acids and nucleotides are abbreviated according to the common international rules (IUPAC). 


\section{INTRODUCTION}

\section{I.1 RNA interference}

The completion of the human genome sequence in 2001 (Venter et a., 2001, The international human genome mapping consortium, 2001) has shifted the focus of interest from sequencing to the understanding of gene function and regulation. Inhibition of individual genes or their products is one way to determine function. One approach to unravel gene function, in particular in vertebrate systems such as man or mouse, is by reverse genetics or loss of function studies. The central dogma of molecular biology, defining the pathways of inherited information from gene to function shows three possible points of attack for gene inhibition. The first is the DNA, which needs to be replicated to maintain genetic information, but is also transcribed to RNA, that serves as a template for protein synthesis in translation. Gene supression is possible by disrupting the coding DNA, inhibition of the RNA or by blocking encoded proteins. Gene knockouts in transgenic mice, inhibition of protein function by antibodies against the gene product of interest (immunodepletion) or antisense technologies for inhibition of translation have yielded valuable information about genes and their function. However, all three techniques also have disadvantages, e.g. they are very cost and labor intensive, they are time consuming and they are not always reliable.

A novel and very promising technique is gene supression by posttranscriptional gene silencing in the presence of double stranded (ds) RNA molecules. The highly conserved mechanism of posttranscriptional gene silencing is referred to as RNA interference (RNAi) (Fire et al., 1998, Hamilton and Baulcombe, 1999, Zamore et al., 2000, Elbashir et al., 2001, Sijen et al., 2001). This process was 
first demonstrated by injection of double-stranded (ds) RNA molecules into Caenhorabditis elegans and subsequent sequence-specific silencing of a homologous target gene (Fire et al., 1998). A close relation to the previously described gene inactivation pathways in certain fungi and plants (reviewed by Bernsstein et al., 2001) was realized and gave rise to the idea of a general conserved mechanism of highly specific ssRNA degradation. Since long dsRNA molecules also proved highly effective in inducing RNAi in the fruit fly Drosophila melanogaster (Hammond et al., 2000) effort was invested into using long dsRNA for gene silencing in vertebrate cells. These attempts were largely unsuccessful, resulting from the fact that long dsRNA molecules activate the vertebrate interferon response. This complex defence system leads to a global inhibition of gene expression by activation of dsRNAdependent protein kinase (PKR) by binding to dsRNA. Any RNA bound is then cleaved in a localised but not sequence specific manner (Williams et al., 1997, Sen, 2001). In addition unspecific mRNA degradation is induced by activation of $2^{\prime}-5^{\prime}-$ oligoadenylate synthase, which in turn activates RNase I (Stark et al., 2001). Nonvertebrate species, however, lack these mechanisms. Although long dsRNAs appeared not to induce RNAi in vertebrates key components of the RNAi pathway were found to be conserved. Among these were both the bidendate endoribonuclease Dicer, that participates in RNAi initiation by processing long dsRNAs into 21-23nt short dsRNAs (Bernstein et al., 2001) and one of the argonaut proteins, ago2 (Hammond et al., 2001), recently found to be the endonuclease that cleaves the target mRNA (Liu et al., 2004, Song et al., 2004). Biochemical investigation of the RNAi pathway (see figure 1) revealed as the effector molecule 21-23nt long dsRNAs with 2nt 3' overhangs in in vitro studies with extracts from Drosophila S2 cells (Elbashir et al., 2001a). In turn these small interfering RNAs (siRNA) were inserted into cultured 
mammalian cells and successfully shown to be capable of inducing RNAi without unspecific responses (Elbashir et al., 2001). The first human endogenous gene to be silenced was the nuclear intermediate filament protein lamin A/C. The experiments that made RNAi finally accessible to mammalian cells were carried out in Göttingen, in the institute and in part in the department I am currently working in. Since our department is basically focused on the cytoskeleton, and in particular on the intermediate filament proteins, the first target for RNAi in human cells was lamin A/C. For a more detailed introduction to lamins see I.2.

Numerous human endogenous genes have been functionally characterized by RNAi approaches subsequent to the discovery of RNAi. More than 20 targets were successfully silenced in a first study in our department. A set of human proteins including intermediate filament proteins such as the three nuclear lamins A/C, B1 and B2, the cytoplasmic intermediate filament proteins vimentin and keratin 18, mitosis related proteins such as the kinesin motor protein Eg5, CENP-E, and cdk1 as well as nuclear and structural proteins were knocked down via RNAi in human HeLa cells. The results were summarized in a publication defining new standards for functional gene characterization (Harborth et al., 2001). Moreover, the standard protocols for application of RNAi, for the documentation of knockdown and the opportunities for modifying siRNA were developed and established here in Göttingen in collaboration with Tuschl's group (Elbashir et a., 2001, 2002, Harborth et al., 2001, 2002). 


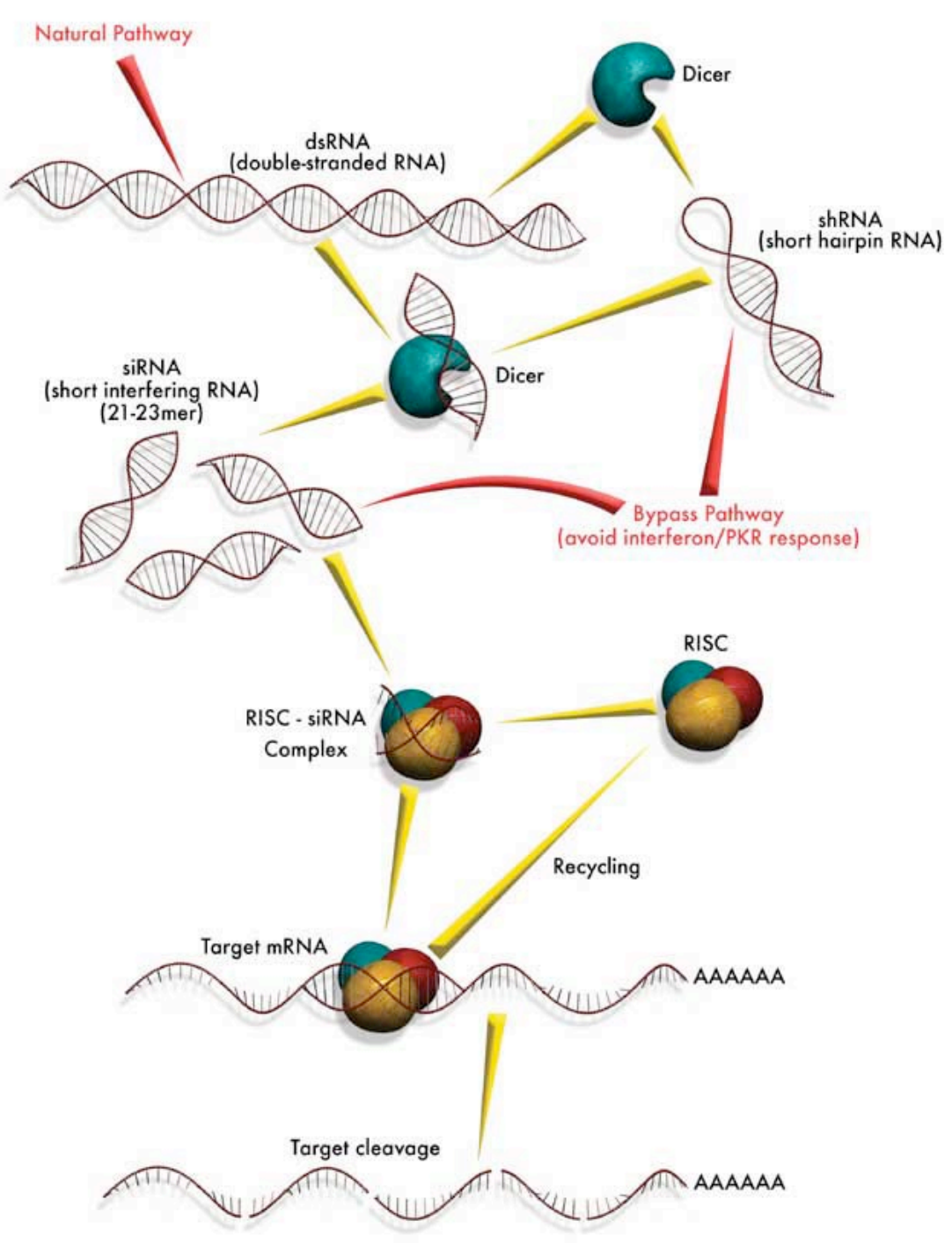

Figure 1: The pathway of RNAi. The endonuclease dicer initiates RNAi by processing long dsRNAs or short hairpin RNAs to $21 \mathrm{nt}$ long dsRNAs with 2 nt $3^{\prime}$ overhangs. Insertion of chemically synthetized siRNAs allows bypassing unspecific effects of the interferon response and PKR activation. The siRNA is then incorporated into RISC and unwound. The antisense strand guides the active RISC to the target position on the target mRNA which in turn is sequence specifically cleaved and degraded. For more details see I.1 and I.5.

(Modified from www.upstate.com/img/pathways/rnai.jpg)

These milestones in functional genetics emerged at the time when I started my doctoral studies, giving me the opportunity to enter a novel and innovative field in molecular life sciences. Thus, I applied RNAi to different targets and successfully characterized the function of a variety of human genes. The results of silencing human 
targets cover the first part of my thesis. These targets included the human endoprotease FACE1, which is involved in posttranslational processing of lamin A/C. Based on the experiences with lamin A/C, FACE1 was an interesting target for RNAi, in particular because it was recently indirectly linked to a severe lamin A related human disease (Hutchinson-Gilford-Progeria-Syndrome, for more details see I.2) (Agarwal et al., 2003). A second human RNAi target was the human coiled coil protein astrin (see 1.3.1). Astrin is a novel mitotic spindle associated protein and as such very interesting for an RNAi approach. A variety of mitosis related proteins, including kinesin related motor proteins and kinetochore related proteins was subjected to RNAi (for more details see I.3.2).

The second part of my thesis shifts from RNAi against human targets to focus on method orientation. Although RNAi was successfully used as a tool to study gene function in numerous studies the mechanism is not yet fully understood and the technique does not always lead to knockdown. Further understanding of the RNAi pathway and technical improvements will make RNA interference an even more powerful tool. Success of an RNAi experiment is based on a variety of factors starting with the design of siRNAs. Therefore, in the second part of my thesis effort was invested in clarifying the requirements for the anatomy of functional siRNAs. Recent publications introduce some basic features associated with efficient siRNAs (Khvorova et al., 2003) and the asymmetric assembly of the RNA induced silencing complex (RISC), that determines which strand of an siRNA is incorporated (Schwarz et al., 2003). Previous studies have shown also that silencing with $5^{\prime}$-phosphorylated single strands is efficient (Martinez et al., 2003). This part of the thesis involves palindromic siRNAs, or siRNAs in which both sense (s) and antisense (as) strands had targets on one gene. The aim of this project was to check, wether silencing 
efficiencies are increased by using homoduplexes of antisense RNAs or by using heteroduplexes of two antisense RNAs targeting the same gene. Furthermore, a dual targeting approach was used. In this approach two antisense RNAs forming a heteroduplex targeting two different genes were delivered into cultured cells. For more details see I.4.

Another factor influencing RNAi is the method of delivery of siRNAs into the target cells. On a population base the silencing efficiency is directly linked to the transfection efficiency. Numerous standard transfection methods were applied successfully. Liposome transfection, electroporation, calcium phosphate precipitation or microinjection were among the methods used to deliver siRNAs into the target cell (for review see Gilmore et al., 2004). A novel alternative technique, i.e. the siRNA delivery by osmotic lysis of pinosomes, is introduced in the fourth part of this thesis (see 1.4).

A further factor is the capability of performing RNAi in particular target tissues or target species. In the fifth and last part of the thesis the opportunities for RNAi based gene knockdown in cultured cells from the zebrafish Danio rerio have been investigated. The motivation for testing RNAi in the zebrafish resulted from conflicting results in the literature. While some labs have reported efficient siRNA mediated gene silencing in zebrafish embryos (Hsieh and Lao, 2003, Love et al., 2004) others report only nonspecific effects (Zhao et al., 2001, Oates et al., 2000). For more details see I.6.

\section{I.2 The human endoprotease FACE1}

Mammals have three genes for nuclear lamins, the structural proteins of the nuclear lamina, that underlies the inner nuclear membrane. The lamina associates with 
chromatin and has been implicated in the regulation of gene expression and in DNA synthesis (for review see (Goldman et al., 2002). Lamin A and the two B lamins, B1 and B2, differ in expression during development and display different behaviours during disassembly of the nuclear envelope prior to cell division. Lamin A is expressed late in development and in some tissues even only postnatally (Broers et al., 1997; Röber et al., 1989). During mitosis lamin A becomes soluble (Gerace and Blobel, 1980), while the B lamins remain bound to membrane vesicles (Goldman et al., 2002; Moir et al., 2000). All three lamins end with a CaaX motif which is subject to a series of post-translational modifications. After farnesylation of the cysteine the three terminal residues are removed by a CaaX protease and the farnesylated cysteine becomes 0 -methylated. Lamin $\mathrm{A}$ is subject to an additional and unique maturation step. The prelamin $\mathrm{A}$ is converted to mature lamin A by a proteolytic conversion. Protein chemical studies indicate cleavage between Tyr646 and Leu647 (Hennekes and Nigg, 1994; Sinensky et al., 1994; Weber et al., 1989). The reason for this maturation is not known, but it explains the solubilization of lamin A in mitosis. Lamin $\mathrm{C}$ is essentially a shortened lamin A form which arises by alternative splicing. It has only 6 unique residues and ends at position 547. Mice lacking the lamin A gene develop normally, but their postnatal growth is severely retarded and they die of muscular dystrophy (Sullivan et al., 1999). Various reports show that the lamin A gene is connected to at least 9 human diseases (reviewed by (Burke and Stewart, 2002) and (Mounkes et al., 2003). Among these laminopathies is also the Hutchinson-Gilford progeria syndrome. For an overview of known mutations in the lamin A sequence leading to laminopathies see figure 2. 


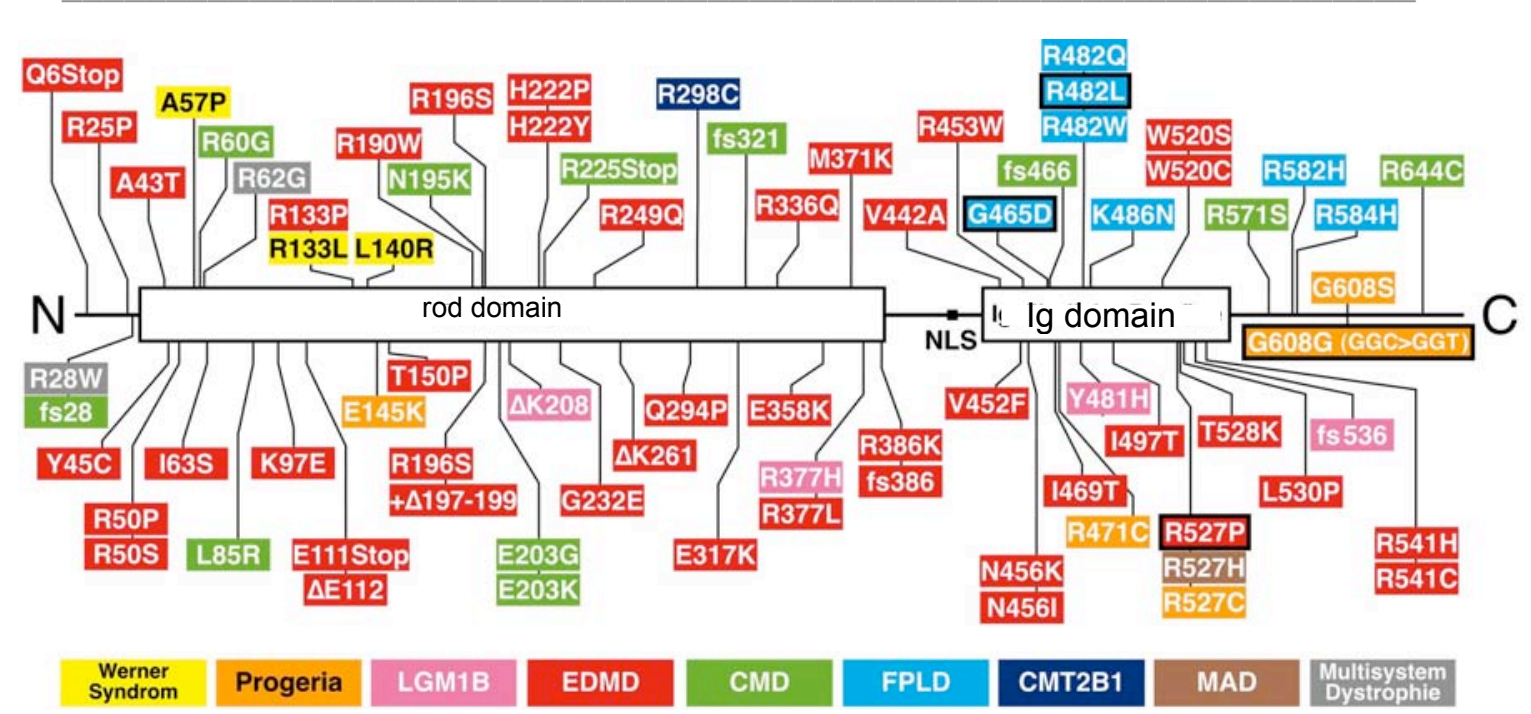

Figure 2: Lamin A mutations related to human diseases. Nine diseases are given, and the individual point mutations are spread throughout the complete lamin A sequence. The diseases are; Werner syndrome (Chen et al., 2003), Hutchinson-Gilford Progeria Syndrome (progeria, de Busk, 1972, Eriksson et al., 2003), Limp-GirdleMuscular-Dystrophy type 1b (LGM1B, Muchir et al., 2000), Emery-Dreyfuss-MuscularDystrophy (EDMD, Emery and Dreyfuss, 1966, Emery, 1989, Di Barletta et al., 2000), congenital muscular dystrophy (CMD), familiar partial lipodystrophy (FPLD, Shackleton et al., 2000, Cao and Hegele, 2000), Charcot-Marie-Toothe-Disease (De Sandre Giovanolli et al., 2002), Mandibuloacrale dysplasia (MAD, Novelli et al., 2002), multi-system-dystrophy (Garg et al., 2002). (Modified from Worman and Courvalin, 2002)

Hutchinson-Gilford progeria syndrome (HGPS) is a rare genetic human disorder characterized by features reminiscent of marked premature aging. In a pioneering analysis Eriksson et al. found a lamin A mutation in 20 of 23 patients with classical HGPS (Eriksson et al., 2003). 18 harbored an identical de novo, not inherited, single base substitution G608G (GGC > GGT) within exon 11. This mutation activates a cryptic splice site within exon 11 resulting in a mutant protein lacking 50 internal amino acid residues (609 to 658 ) before the carboxy-terminal end (658 to 664) with its CaaX motif. A further patient showed a different substitution within the same codon, while one patient revealed a missense mutation in exon 2 (E145K). A simultaneously published, but less extensive study, reported the same mutation G608G in a patient 
suffering from mandibuloacral dysplasia with HGPS features (De Sandre-Giovannoli et al., 2003). Although occasional missense mutations in lamin A are found (Cao and Hegele, 2003; Chen et al., 2003; Eriksson et al., 2003; Novelli et al., 2002), the major change in HGPS is the deletion of 50 aminoacids due to a cryptic splice site analyzed in detail by Eriksson et al. (Eriksson et al., 2003). Since this deletion removes the cleavage site for the conversion of prelamin A to lamin A the mutated prelamin A may stay permanently in the farnesylated form.

Defective prelamin A processing has been clearly demonstrated in mouse knockouts of the ZMPSTE24 metalloproteinase (Bergo et al., 2002; Pendas et al., 2002). ZMPSTE24 is an ortholog of the yeast enzyme STE24, that is involved in the processing of mating pheromone $a$-factor, a short peptide ending in a farnesylated, O-methylated cysteine (Tam et al., 1998). Homozygote knockouts of the murine gene have a phenotype resembling that of HGPS patients including growth retardation and premature death from cardiac dysfunction and alopecia. Cells from such mice lack mature lamin A and show instead prelamin A (Bergo et al., 2002; Pendas et al., 2002). Thus murine ZMPSTE24, and its human ortholog FACE1, may indeed be the proteases involved in lamin A processing. In line with this view the human enzyme expressed in yeast mutants complements a-factor synthesis (Agarwal et al., 2003; Tam et al., 1998). Finally a study of 4 patients suffering from mandibuloacral dysplasia associated with progeria identified 1 patient with defects in ZMPSTE24 in the absence of lamin A mutations (Agarwal et al., 2003).

In this part of my thesis a functional characterization of the human endoprotease FACE1 by siRNA mediated gene silencing is presented. Silencing FACE1 in HeLa cells leads to accumulation of prelamin A at the nuclear lamina and to a stop in cell division. One population of FACE1 silenced cells arrest in aberrant 
mitosis and later enter apoptosis. The second population show abnormalities in nuclear morphology including micronuclei formation. In contrast when FACE1 was silenced in HeLa cells lacking lamin A, FACE1 silencing results in no mitotic arrest and the cells appear normal. The striking similarities seen between the results described here for FACE1 silenced HeLa cells and other results on HutchinsonGilford patients where certain mutations in the lamin A molecule prevent cleavage by the FACE1 enzyme are discussed.

\section{I.3 Mitosis related targets for RNAi}

\section{I.3.1 Eukaryotic cell division}

The mitotic spindle, a bipolar microtubule based structure, is responsible for accurate chromosome segregation during mitosis (Compton, 2000). The basic structural element of the spindle is an antiparallel array of microtubules with their minus ends anchored at the spindle poles and their plus ends projecting towards the chromosomes. This polar lattice of microtubules serves as a track for motors of the dynein and kinesin superfamily (Hirokawa et al., 1998a; Kim and Endow, 2000). Spindle microtubules are highly dynamic structures with a half life of 60-90 sec and this dynamic instability is fundamental to mitotic spindle structure and regulation (Joshi, 1998; Saxton et al., 1984). Several tubulin binding proteins promote microtubule depolymerization whereas other microtubule associated proteins (MAPs) counteract the destabilizing effect and some evidence suggests that cell cycle dependent regulation of these two protein families by cyclin-dependent kinase 1 is involved in regulating the fast turnover of mitotic microtubules compared to interphase microtubules (Tournebize et al., 2000; Vasquez et al., 1999; Wittmann et al., 2001). Microtubule motor proteins have an important function in spindle 
organization. These proteins have been devided into two classes, the kinesin superfamily, which includes both plus- and minus-end directed motors and the minusend directed motor protein dynein. Different kinesins cooperate and counteract during the process of spindle assembly. Oligomeric motor complexes that can cross-link and move along microtubules have been shown to be sufficient for self organization of tubulin asters in vitro (Heald et al., 1996; Karsenti and Vernos, 2001; Nedelec et al., 1997; Walczak et al., 1998; Wittmann et al., 2001).

The primary function of the mitotic spindle is to segregate chromosomes such that a complete set of chromosomes ends up at each spindle pole. The process of segregation depends on a complex interplay between forces generated by motor proteins associated with spindle microtubules, kinetochores and chromosome arms as well as dynamic instability of spindle microtubules (Howell et al., 2001; Maney et al., 2000; Nicklas et al., 1995; Rieder et al., 1995; Rieder and Salmon, 1998). Chromosome separation also depends on attachment of chromosomes to spindle microtubules via their kinetochores and it has been shown that cytoplasmic dynein as well as several kinesin family members localize at the kinetochore (Banks and Heald, 2001). CENP-E is another kinesin-like protein that is part of a kinetochore associated signalling pathway that monitors kinetochore-microtubule attachment and ensures high segregation fidelity. Depletion of CENP-E from mammalian kinetochores leads to a reduction of kinetochore-microtubule binding and mitotic arrest with a mixture of aligned and unaligned chromosomes (Abrieu et al., 2000; Lombillo et al., 1995; Yao et al., 2000; Yen et al., 1992). 


\section{I.3.2 The human coiled coil protein astrin}

In order to gain more insight into spindle organization Mack and Compton (2001) used mitotic microtubules prepared from HeLa cell extracts to identify spindle associated proteins in an elegant mass spectroscopic analysis. Several proteins with known functional roles in spindle assembly and a novel non-motor coiled coil protein named astrin were described. Using immunofluorescence and ectopic expression of the GFP-tagged astrin its spindle localization during mitosis was confirmed (Mack and Compton, 2001). Jörg Schnabel had earlier identified an astrin cDNA clone in a two-hybrid screen with a keratin 18 bait although this interaction could not be confirmed using other methods. The full-length $3,793 \mathrm{bp}$ cDNA was named DEEPEST because the predicted protein contains this sequence motif. Antibodies located the protein to the spindle and sequence predictions indicated two long coiled coils. When the cDNA sequence was entered into the EMBL/GEN Bank in May 1998 information that DEEPEST was a coiled coil protein associated with the mitotic spindle apparatus (accession number AF 063308) was added. Due to a sequence error at position 3341 the predicted protein sequence lacked the C-terminal 101 amino acids provided by Mack and Compton (accession number AF 399910). An identical protein sequence to that provided by Jörg Schnabel for DEEPEST was provided by Chang et al. (2001), (accession number P33176 and NM 006461) who called this protein hMAP126. hMAP126 has been described as a mitotic spindle associated protein that is post-translationally modified by cdk1 phosphorylation. To avoid confusion we have dropped the name DEEPEST and use instead the name astrin.

Here I provide a more detailed characterization of astrin's domain organization and function in spindle pole organization (Gruber et al., 2002, Gruber, 2002)). Astrin 
has a domain structure resembling that of motor proteins with a large head domain, which however lacks sequence similarity to motor domains, and a coiled coil domain responsible for formation of parallel dimers. Under physiological conditions recombinant astrin dimers oligomerize via their head domains into aster like structures. Moreover, I show that astrin is essential for progression through mitosis. Depletion of astrin by RNA interference results in the formation of multipolar and highly disordered spindles and lead to growth arrest and apoptotic cell death. These results indicate that astrin has a critical role in assembly or orientation of the bipolar structure of the spindle.

\section{I.4 Rational design of functional siRNAs}

According to the generally accepted model of RNAi biochemistry a long dsRNA (>30nt) is processed by the endonuclease dicer to form small interfering RNAs (21 nt long dsRNA with 2 nt 3' overhangs). The siRNAs have 5'-phosphate and 3'-hydroxyl groups (Elbashir et al., 2001a) and therefore display the pattern of RNase III cleavage products. The RNase III like enzyme dicer contains helicase and PAZ domains as well as two dsRNA binding sites (Bernstein et al., 2000, Ketting et al., 2001). Long dsRNA cleavage and the rest of the RNAi pathway takes place in the cytoplasm of cells (Hutvagner et al., 2002, Zeng et al., 2002). The siRNAs are incorporated into an RNA induced silencing complex (RISC). Incorporation into RISC requires 5'-phosphate groups (Nykanen et al., 2001) and chemically synthesized siRNAs are rapidly phosphorylated by an endogenous kinase (Schwarz et al., 2002). Only one strand of the siRNA, which is unwound during RNAi initiation, is incorporated into RISC. The antisense strand, which is perfectly complementary to the target mRNA guides RISC to its target, which is subsequently cleaved at a single 
position $10 \mathrm{nt}$ from the $5^{\prime}$ end of the siRNA (Elbashir et al., 2001a). Functional RNAi depends on a variety of factors, and some of these concern sequence determinants intrinsic to the siRNA. The assembly of RISC is asymmetric and depends on the side from where the helicase attacks the siRNA. The 5'end determines, which strand goes into RISC and the choice is made by the lower basepairing energy (Schwarz et al., 2003). Thus, the design of an siRNA may dramatically influence its functionality. A very detailed study on efficient siRNA anatomy resulted in a set of rules, which may help to design molecules that are successful in RNAi (Khvorova et al., 2003). The basic requirements are summarized in Figure 3.

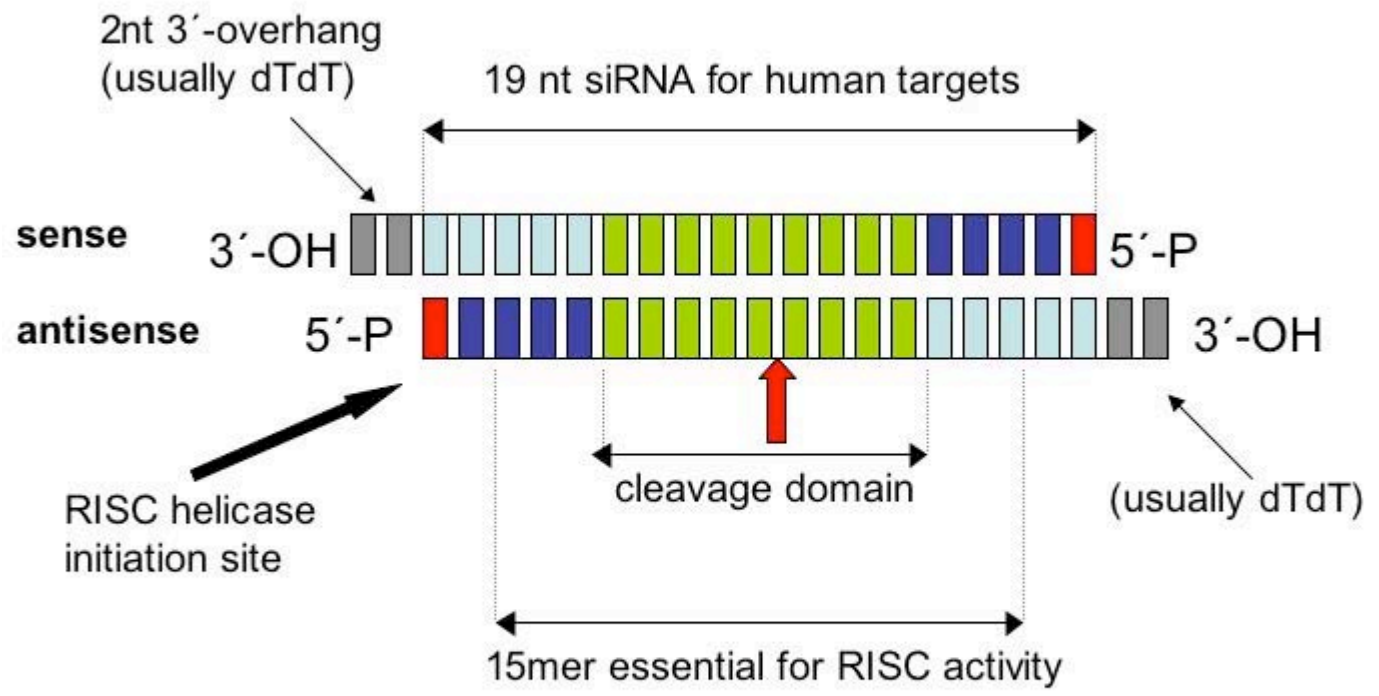

Figure 3: Anatomy of a synthetic $21 \mathrm{nt}$ siRNA with the essential structural requirements for activity. The classic 19 basepair 2 nt 3 '-overhang (usually dTdT) siRNA is shown. The $19 \mathrm{bp}$ core duplex is effective for gene silencing in mammalian cells (Czauderna et al., 2003) and a $15 \mathrm{bp}$ central region appears to be essential for RISC activity (Martinez and Tuschl, 2004). The 5'-end (preferably of the antisense strand) with lower basepairing energy determines the site of helicase initiation (Schwarz et al., 2003, Khvorova et al., 2003). Competing 5'-end of sense and antisense strands are highlighted in red. Therefore, the antisense $5^{\prime}$-end appears to be most sensitive to terminal modifications (Dorsett and Tuschl, 2004). 
The antisense strand was shown to guide RISC to its target and thereby mediate RNAi. Transfection of single stranded antisense molecules was shown to be efficient in inducing RNAi (Martinez et al., 2002).

Here I provide data on RNAi against lamin $\mathrm{A} / \mathrm{C}$ by transfection of imperfect RNA duplexes. Three approaches were followed. The first was based on self complementary antisense RNAs that form homodimeric dsRNA. In the other two approaches two asRNAs that were complementary to each other were selected and tested by transfection into HeLa cells to see if they could mediate RNAi.

\section{I.5 siRNA delivery by osmotic lysis of pinosomes}

The delivery of nucleic acids into mammalian cells is referred to as transfection. This procedure is crucial for a broad variety of applications, including the overexpression of proteins, the expression of fusion proteins or the establishment of knockout constructs. In particular functional gene characterisation using posttranscriptional gene silencing via RNA interference demands effective transfection procedures without side effects and without cytotoxicity. The usual procedure to transfect small interfering RNAs (siRNAs) (1) or small hairpin RNA (shRNA) encoding constructs (2) into cells uses cationic liposomes. These form a liposomenucleic acid complex and serve as the shuttle vector to get the small RNAs into the cytoplasm. The RNA constructs mediate RNA interference by site specific mRNA degradation.

The aim of this part of the thesis was to find a method to transfect cultured mammalian cells with small interfering RNAs that did not involve the use of transfection reagents. Here I found that the technique of loading cells based on the 
pinocytotic uptake of hypertonic media mixed with the compound to be loaded (3) can also be used to get siRNAs into cells. Once cells have taken up the siRNAs in pinocytotic vesicles, the vesicles are lysed by a shock in hypotonic medium that results in release of the compound into the cytosol. The pinocytotic vesicles do not fuse with lysosomes (4) and therefore store their cargo until osmotic lysis is forced. The pinocytosis technique, already established in the early 1980s by Okada \& Rechsteiner (3), has been mainly used for the delivery of proteins or fluorescent dyes into the cells. Here I report the first application of pinocytosis mediated transfection to deliver biologically active nucleic acids to cultured mammalian cells for use in RNA interference studies.

\section{I.6 RNA interference in the zebrafish (Danio rerio)}

The major part of this study involves RNA interference as a research tool to unravel gene functions in mammalian systems. The effect of sequence specific gene silencing in presence of dsRNA molecules was discovered originally in C. elegans (Fire et al., 1998). Those results have stimulated an increasing number of approaches to establish RNAi mediated gene knockdown in non mammalian model organisms. The zebrafish (Danio rerio) is of special interest since it is a well established model in which to study developmental processes. By employing gain and loss of function techniques it is possible to obtain insights into the roles of both wild-type and heterologously expressed genes (for review see Key and Devine, 2003).

Lim et al. (2002) have reported the presence of dicer mRNA and the active enzyme in the fertilized egg and the early embryo of the zebrafish while Wienholds et al. (2002) have shown that dicer is essential for embryonic development. Dicer knockout fish develop normally but die on day 14 . If the maternal pool of dicer 
mRNA is cut down, fish survive only until day 11 . These results suggest an essential function of dicer only after the eleventh day of development, and also indicate the presence of a functional micro RNA / RNAi pathway. These results conflict with the situation in organisms, such as the fruitfly Drosophila melanogaster or the mouse Mus musculus, where micro RNAs are expressed at very early stages of embryonic development (Lagos-Quintana et al., 2001, 2002) and essentially regulate gene expression.

A number of groups have attempted to establish RNAi mediated gene silencing in zebrafish. Conflicting data is reported in the literature. Several groups report specific effects after delivery of dsRNA molecules into fish embryos. Thus, a specific reduction of exogenous GFP fusion proteins was observed after the introduction of the corresponding siRNAs in embryos of the rainbow trout (Boonanuntansarn et al., 2003), while Hsieh and Liao reported specific silencing of the zebrafish M2 muscarinic acetylcholine receptor in the developing embryo after injection of dsRNA targeting the M2 mRNA (Hsieh and Liao, 2003). Silencing of endogenous genes via injection of specific siRNAs into the yolk of two-cell zebrafish embryos was reported (Dodd et al., 2004). In contrast, other laboratories found only nonspecific effects after injection of long dsRNAs and siRNAs into the embryo. A variety of defects were observed after injection of either type of dsRNA (Zhao et al., 2001, Oates et al., 2000) independent from target genes. A possible explanation for the nonspecific defects that were observed in the presence of double stranded RNA might be an interferon response in the fish. The interferon 1 pathway is activated in the presence of dsRNA (Collet and Secombes, 2002) and increased interferon levels have been reported in the presence of dsRNA in salmon embryos (Jensen et al., 2002). Since all groups 
attempted to target different genes, exogenous and endogenous, and reported conflicting results one question remains to be answered. Can the zebrafish do RNAi?

RNAi in zebrafish has also been studied in the independed research group for germ cell development in zebrafish led by Dr. Erez Raz in our institute. Zebrafish embryos were injected with a variety of dsRNAs. These included long dsRNA (>250bp), short hairpin RNAs (shRNA) and siRNA, all of which have been shown previously to induce RNAi in a variety of organisms. Targets included endogenous genes as well as exogenously GFP. SiRNAs were designed to target GFP or endogenous genes. Hairpin RNAs with the corresponding siRNA sequences were generated to allow complete dsRNA processing, including the initial cleavage by dicer. Injections involving 10 unmodified siRNAs as well as 10 hairpin precursors, targeting 5 different genes, did not result in characteristic mutant or morphant phenotypes. Increasing the amount of RNAs produced malformed embryos as observed in the calibration experiments using dsRNA concentrations from 10 to 200 $\mu \mathrm{M}$ in the injection buffer. Interestingly, high concentrations $(>50 \mu \mathrm{M})$ of siRNA oligos caused malformations. In two cases, when targeting the genes FLH and SPT, the resulting phenotype resembled to some extent the phenotype observed after morpholino based silencing of the FLH and SPT. However, similar phenotypes were observed in embryos injected with control oligos or with those targeting different genes, implying non-specific effect of the RNAi treatment. Concentrations higher than $100 \mu \mathrm{M}$ siRNA in the injection buffer caused lethality (unpublished data from Raz'group). The observed effects seemed to be independent of target specificity and length of the dsRNA, since the long dsRNA molecules (300nt) as usually used for RNAi in C. elegans, also induced unspecific effects. Injection of single RNA strands (e.g. mRNA) did not cause the unspecific effects described above. 
Both siRNAs and shRNAs targeting mGFP were evaluated for their RNAi functionality in cultured human HeLa cells, an established system for RNAi in cell culture. siRNA or shRNA injected into the yolk of early (2-4 cell) zebrafish embryos did not lead to specific gene knockdown. An uptake of siRNAs from the yolk into the cells was confirmed by injection of FITC labelled siRNAs and subsequent fluorescence video microscopy. A weak reduction of the appropriate target was generally observed, but always combined with nonspecific effects on the gene expression of foreign genes and an altered morphology of the developing embryo.

The results obtained in other labs on the zebrafish embryo indicated the necessity of using an alternative system to assess RNAi in the zebrafish. Since there is no method to deliver nucleic acids homogenously into the body of adult zebrafish I have used cultured zebrafish cells of adult and embryonic origin for RNAi experiments. With different siRNA delivery techniques I successfully knocked down lamin A and lamin B2, and also exogenously expressed GFP. These experiments showed functional RNAi in zebrafish cells of adult and embryonic origins without the unspecific effects that were observed in experiments with fish embryos. 


\section{MATERIALS AND METHODS}

\section{II.1 Materials}

\section{II.1.1 Chemicals and Enzymes}

Suppliers of the chemicals and enzymes used in this study included Sigma (Taufkirchen), Roche Diagnostics (Mannheim) or Boehringer (Mannheim) and usually were of molecular biology quality. Enzymes, in particular restriction endonucleases and Taq or pfu DNA polymerase were from MBI Fermentas (St. Leon-Rot, France) or NEB (New England Biolabs, Boston, MA), respectively. Other enzymes used were mainly components from commercial kits and are described in the Methods section.

\section{II.1.2 siRNA and nucleotides}

RNA oligonucleotides were chemically synthesised in the Group for combinatorial biochemistry (Dr. T. Tuschl, AG 105, Max-Planck Institute for biophysical Chemistry, Göttingen) or commercially supplied by Dharmacon (Lafayette, Colorado). Nucleotides (dNTPs) for PCR were from Boehringer (Mannheim), PCR primers were from NAPS (Göttingen).

\section{II.1.3 Machines}

Centrifuges

Eppendorf 5415C (Hamburg)

Variofuge RF, Heraeus Sepatech (Osterode)

Sorvall RC-5 (GSA or SS34 rotor), DuPont (Bad

Nauheim)

Photometer

Ultraspec 2000, Pharmacia Biotech (Freiburg)

PCR thermocycler Perkin Elmar PCR 2400 (Überlingen)

FPLC Amersham Pharmacia Biotech (Freiburg) with Mono-Q ion exchange columns 
Tankblot chamber

Flow cytometer

Cell counter

CellScreen
Trans-Blot Cell, BioRad (Munich)

FACScan (BD Bioscience, Pharmingen, Wisconsin)

CASY counter Model TT, Schärfe systems ( Reutlingen)

Innovatis (Bielefeld)

\section{II.1.4 Buffers and Solutions}

$\begin{array}{llll}\text { PBS: } & \mathrm{NaCl} & 137 \mathrm{mM} & \mathrm{pH} 7.1 \\ & \mathrm{Na}_{2} \mathrm{HPO}_{4} & 7 \mathrm{mM} & \\ & \mathrm{KH}_{2} \mathrm{PO}_{4} & 1.5 \mathrm{mM} & \\ & \mathrm{KCl} & 2.7 \mathrm{mM} & \\ & & & \\ \text { TBS: } & \text { Tris-HCl } & 20 \mathrm{mM} & \mathrm{pH} 7.4 \\ \text { TBST: } & \mathrm{NaCl} & 150 \mathrm{mM} & \\ & \mathrm{TBS} & +0.2 \% \text { Tween20 } & \\ \text { TE buffer: } & \text { Tris-HCl } & 10 \mathrm{mM} & \mathrm{pH} 8.0 \\ & \text { EDTA } & 1 \mathrm{mM} & \\ \text { TBE-buffer: } & \text { Tris-HCl } & 1.78 \mathrm{M} & \mathrm{pH} 8.0 \\ (20 \mathrm{x}) & \text { Boric acid } & 1.78 \mathrm{M} & \\ & \text { EDTA } & 0.5 \mathrm{M} & \end{array}$

These are the standard buffers. Other buffers are described in Method section.

\section{II.1.5 Media and Culture Plates}

LB medium: $\quad$ bacto tryptone $10 \mathrm{~g}$

bacto yeast extract

$\mathrm{NaCl}$

$5 \mathrm{~g}$

$\mathrm{H}_{2} \mathrm{O}$

$10 \mathrm{~g}$

to 1 litre

SOB medium: $\quad$ bacto tryptone $20 \mathrm{~g}$ (1 litre bacto yeast extract $\mathrm{NaCl}$

$\mathrm{KCl}$

$5 \mathrm{~g}$

$\mathrm{H}_{2} \mathrm{O}$

$0.5 \mathrm{~g}$

$186 \mathrm{mg}$

$\mathrm{MgCl}_{2}, 2 \mathrm{M}$ to 1 litre $5 \mathrm{ml}$ (added after autoclaving)

SOC medium: $\quad$ SOB medium $+10 \mathrm{ml}$ per litre $50 \%$ Glucose in water. 
Agar plates: $15 \mathrm{~g}$ Bacto agar (Gibco-BRL, Eggenstein) per litre of medium was added before autoclaving the medium.

Cell culture medium: low glucose DMEM (Gibco-BRL, Eggenstein) supplemented with $10 \%$ FCS (Boehringer, Mannheim). For routine cell culture this medium is supplemented with penicillin $(100 \mathrm{U} / \mathrm{ml})$ and streptomycin $(100 \mu \mathrm{g} / \mathrm{ml})$

Transfection medium: $\quad$ low glucose DMEM $+10 \% \mathrm{FCS}$, free of antibiotics

\section{II.1.6 Bacterial Host Strains}

Strain

XL1 blue proAB, lacI ${ }^{\mathrm{q}} \mathrm{ZDM} 15, \mathrm{Tn} 10\left(\right.$ tet $\left.\left.^{\mathrm{R}}\right)\right\}$

growth in LB medium with $12.5 \mu \mathrm{g} / \mathrm{ml}$ tetracycline

BL21(DE3)pLysS: E. coli B, $\mathrm{F}^{-}$, dcm, ompT, hsdS, $\left(\mathrm{r}_{\mathrm{B}^{-}}, \mathrm{m}_{\mathrm{B}}{ }^{-}\right)$, gal, lambda(DE3)\{pLysS, $\left.\mathrm{cam}^{\mathrm{R}}\right\}$, growth in LB medium with $30 \mu \mathrm{g} / \mathrm{ml}$ chloramphenicol (Stratagene, Heidelberg)

\section{II.1.7 Vectors}

PCR products (see II.2.4) were cloned into the TOPO T/A Cloning vector, which is a component of the standard T/A cloning kit from BD Biosciences (Heidelberg). For expression of the recombinant astrin protein the insert was subcloned into the bacterial expression vector pET23a (Invitrogen, Heidelberg).

TOPO TA cloning vector pCR2.1

pBAD expression vector (Invitrogen) 


\section{II.1.8 Cell lines}

The following cell lines were kindly provided by Prof. Mary Osborn (MPI for biophysical Chemistry):

cell line $\quad \underline{\text { source }}$

HeLa SS6: human cervix adenocarcinoma (ATCC and Gey et al., 1952)

MCF-7: human breast adenocarcinoma (ATCC and Soule et al., 1973)

HEK293 human embryonic kidney (ATCC)

HL60 human (ATCC)

Glioma line U333 CG/343 MG

SW3T3 mouse embryonic fibroblast (ATCC)

The following cell lines were purchased from ATCC:

SDJ zebrafish (ATCC)

ZFL $\quad$ zebrafish (ATCC)

Z4F zebrafish embryonic fibroblast (ATCC)

Cell lines were maintained in DMEM supplemented with 10\% FCS and antibiotics (100 $\mathrm{U} / \mathrm{ml}$ penicillin and $100 \mu \mathrm{g} / \mathrm{ml}$ streptomycin).

\section{II.1.9 Software}

For analysis of DNA, RNA or protein sequences different softwares were used:

JellyFish (Biowire)

MacDNAsis

Mac DNAstar

Online databases and web tools for bioinformatical calculations are given in the methods section. 


\section{Materials and Methods}

\section{II.2. Molecular biology methods}

\section{II.2.1 Purification of E. coli plasmids for analysis (Miniprep)}

Bacteria were grown on agar plates containing a selective antibiotic, e.g. penicillin or kanamycin. A single clone or colony was picked with a sterile pipette tip and inoculated in $4 \mathrm{ml}$ of liquid medium with the same antibiotics. After overnight incubation on a shaker at $37^{\circ} \mathrm{C} 2 \mathrm{ml}$ of this culture were harvested by centrifugation (Eppendorf Variofuge) at $6500 \mathrm{rpm}$ for $5 \mathrm{~min}$ at room temperature. The bacterial pellet was resuspended in $150 \mu 1$ TELT buffer and mixed with $15 \mu 110 \mathrm{mg} / \mathrm{ml}$ chicken egg lysozyme (Serva, Heidelberg) and incubated for $5 \mathrm{~min}$ at room temperature. Samples were incubated in boiling water for 2 min and subsequently cooled on ice to room temperature. Cell fragments were pelleted by centrifugation at $13,000 \mathrm{rpm}$ at $4^{\circ} \mathrm{C}$ for $15 \mathrm{~min}$. The flocculent debris was removed with a sterile toothpick. Then plasmid DNA was precipitated with 0.7 volumes $(115 \mu 1)$ of 2-propanol and centrifuged as above. The DNA pellet was washed twice with $500 \mu 170 \%$ ethanol, centrifuged again, and dried with a vacuum centrifuge (SpeedVac Concentrator, Savant, Rockville). Lyophilised DNA was resuspended either in $30 \mu 1 \mathrm{TE} / \mathrm{RNAse}$ or double distilled water.

$\begin{array}{llll}\text { TELT buffer: } & \text { Tris-HCl } & 50 \mathrm{mM} & \mathrm{pH} 8.0 \\ & \text { EDTA } & 62.5 \mathrm{mM} & \\ & \text { LiCl } & 2.5 \mathrm{M} & \\ \text { Triton X-100 } & 0.4 \%(\mathrm{v} / \mathrm{v}) & & \\ \text { TE/RNAse: } & \text { Tris-HCl } & 10 \mathrm{mM} & \mathrm{pH} 7.5 \\ & \text { EDTA } & 1 \mathrm{mM} & \\ & \text { RNAse A } & 0.1 \mu \mathrm{g} / \mathrm{ml} & \\ & \text { RNAse T1 } & 0.1 \mu \mathrm{g} / \mathrm{ml} & \end{array}$




\section{II.2.1.1 Preparative Plasmid Purification (Midiprep)}

For preparative purposes $200 \mathrm{ml}$ of liquid medium were inoculated with $0.5 \mathrm{ml}$ of an overnight preculture (see II.2.1) and incubated overnight at $37^{\circ} \mathrm{C}$ on a shaker. Isolation of plasmid DNA was performed with an ion exchange chromatography kit (QiaFilter Plasmid Midi Kit, Qiagen, Hilden) for DNA amounts up to $500 \mu \mathrm{g}$. Experimental procedures were applied as described in the manufacturer's handbook, using buffers and equipment contained in the kit. Plasmid DNA was precipitated with 2-propanol and centrifuged for $30 \mathrm{~min}$ at 15,000 rpm. After washing the plasmid pellet with $10 \mathrm{ml} 70 \%$ ethanol and recentrifugation the DNA was dried and subsequently resuspended in $200 \mu 1$ sterile $\mathrm{H}_{2} \mathrm{O}$.

\section{II.2.2 $\quad$ Agarose Gel Electrophoresis}

Agarose gel electrophoresis was performed for analytical and preparative purposes. Size and amount of DNAs were rapidly determined using this method and bands of particular sizes could be cut out and isolated from the gel (II.2.3). The standard composition of gels for DNA fragments from 0.5 to $5 \mathrm{kBp}$ was $1 \%$ agarose in $1 \mathrm{x}$ TBE buffer with $0.4 \mu \mathrm{g} / \mathrm{ml}$ ethidium bromide for visualisation of DNA bands under UV light. The standard marker was a $1 \mathrm{~kb}$ ladder (GibcoBRL). DNA samples were 5:1 diluted with 5x sample buffer (Bluejuice, GibcoBRL) and 5 to $10 \mu 1$ were used for analytical gels while $50 \mu \mathrm{l}$ were used for preparative gels. Electrophoresis was run at $100 \mathrm{~V}$ at room temperature with $1 \mathrm{x}$ TBE as running buffer. Analysis and photography was performed under UV light with a wavelength of $312 \mathrm{~nm}$. 


\section{II.2.3 DNA-elution from agarose gels}

After preparative restriction digestion of DNA, the fragments were separated by electrophoresis and the band of correct size was cut from the gel under UV light (366nm wavelength). Elution from the gel piece was done with the QIAquick Gel Extraction Kit (Qiagen) following the manufacturer's protocol. The resulting purified DNA was redissolved in $40 \mu \mathrm{TE}$ buffer and stored at $-20^{\circ} \mathrm{C}$.

\section{II.2.4 Polymerase Chain Reaction}

PCR is the method of choice for in vitro amplification of DNA (Mullis et al., 1987). Utilisation of oligonucleotides (primers) allows choice of particular DNA sequences to be amplified. The PCR is basically a cyclic reaction chain consisting of three steps: 1. melting of dsDNA (denaturation), 2. primer annealing, 3. synthesis of new DNA strands by DNA polymerase (primer extension)

\section{II.2.4.1 $\quad$ Primer design}

The target sequence (in this case the full length ORF of astrin, GeneBank A7399910) was checked for restriction sites, start and stop codon position and translated to the primary amino acid sequence with a sequence analysis software (JellyFish, www.biowire.com). The sense and antisense primers (see below) were designed to flank the complete translated cDNA sequence of the astrin ORF and contained extra restriction sites for subsequent subcloning of the PCR product into appropriate multicopy or expression plasmids (Itakura et al., 1984). The integration of additional restriction sites into the native target sequence is referred to as mutagenesis-PCR. 
Primers for astrin PCR:

astr-1 (5'-primer, sense):

5'- CCG TCG ACA TGT GGC GAG TGA AAA AAC TGA GC-3'

$\mathrm{T}_{\mathrm{m} \text { (basic) }}=66^{\circ} \mathrm{C}$ resp. $\mathrm{T}_{\mathrm{m} \text { (salt adjusted) }}=61^{\circ} \mathrm{C}$, Sall site in bold

astr-2 (3'-primer, antisense):

5'-CCC TCG AGT CAG AAA TTC CAG CAA TCC CTG TAG-3'

$\mathrm{T}_{\mathrm{m} \text { (basic) }}=66^{\circ} \mathrm{C}$ resp. $\mathrm{T}_{\mathrm{m} \text { (salt adjusted) }}=61^{\circ} \mathrm{C}$, XhoI site in bold

(temperatures calculated at: http://www.promega.com/biomath/default.htm\#melt)

\section{II.2.4.2 Mutagenesis PCR for astrin amplification}

Mutagenesis PCR was used for amplification of the protein coding area of astrin cDNA and parallel addition of restriction sites on the $5^{\prime}$ and $3^{\prime}$ ends.

The standard cycler protocol for $50 \mu 1$ final volume was:

$5 \mu 1$ 10x PCR buffer, $5 \mu 1 \mathrm{dNTP}$ mix ( $2.5 \mathrm{mM}$ each nucleotidetriphosphate), $2 \mu 1$ template DNA (HeLa ZapII cDNA library), $1 \mu 1$ sense primer (100 pM), $1 \mu 1$ antisense primer (100 pM), $1 \mu 1$ Pfu polymerase (3 U), $32.5 \mu 1 \mathrm{H}_{2} \mathrm{O}, 2.5 \mu 1$ DMSO.

temperature protocol:step 1 (initial denaturation): $5 \mathrm{~min}, 95^{\circ} \mathrm{C}$ step 2 (denaturation): $1 \mathrm{~min}, 95^{\circ} \mathrm{C}$

step 3 (primer annealing): $1.5 \mathrm{~min}, 62^{\circ} \mathrm{C}$

step 4 (primer extension): $8 \mathrm{~min}, 72^{\circ} \mathrm{C} \rightarrow$ steps 2 to 4 repeated for 35 times step 5 (final elongation): $15 \mathrm{~min}$ at $72^{\circ} \mathrm{C}$ 
A HeLa cDNA library (HeLa Lamda ZapII, Stratagene, Heidelberg) was used as template DNA. Times given in the temperature protocol depended on the melting temperature of the primers for step 3 and the length of the expected product (step 4). Pfu polymerase synthesises $1 \mathrm{~kb}$ in $\sim 2 \mathrm{~min}$ and the astrin ORF has a length of $\sim 3.7 \mathrm{~kb} .5 \mu \mathrm{l}$ of the product were applied to analytical gel electrophoresis, the rest was used for preparative restriction digestion (II.2.5).

\section{II.2.5 Restriction digestion of DNA}

Restriction endonucleases, which catalyse hydrolysis of specific DNA sequences are part of a protection mechanism against foreign DNA in bacteria. The sequences recognised by the restriction endonucleases are characteristic for the bacteria strain from which they originate. Here we used type II endonucleases, which recognise particular $6 \mathrm{bp}$ target sequences and cut these producing $3^{\prime}$ or $5^{\prime}$ overhangs, so called sticky ends.

For subsequent ligation into an appropriate vector the DNA was subjected to preparative restriction digestion. For fragment identification and to check insert orientation an analytical digest was performed.

Preparative digest (50 $\mu 1$ final volume):

$25 \mu 1$ of purified PCR product or $20 \mu 1$ of native plasmid solution were used. Composition: $\mathrm{x} \mu \mathrm{l}$ DNA, $5 \mu 110 \mathrm{x}$ buffer (for enzymes A and B), $2 \mu 1$ enzyme $\mathrm{A}, 2 \mu 1$ enzyme $\mathrm{B}, \mathrm{H}_{2} \mathrm{O}$ to a final volume of $50 \mu \mathrm{l}$.

Incubation: overnight (or at least $3 \mathrm{~h}$ ) at $37^{\circ} \mathrm{C}$ followed by enzyme inactivation for $10 \mathrm{~min}$ at $80^{\circ} \mathrm{C}$. 
Analytical digest $(10 \mu 1)$ :

$5 \mu 1$ plasmid DNA, $1 \mu 1$ 10x buffer, $0.5 \mu 1$ enzyme A, $0.5 \mu l$ enzyme $\mathrm{B}, 3 \mu 1 \mathrm{H}_{2} \mathrm{O}$

The solution was incubated for $2 \mathrm{~h}$ at $37^{\circ} \mathrm{C}$ and examined by analytical agarose gel electrophoresis.

\section{II.2.6 Ligation reactions}

Ligases catalyse the energy dependent covalent binding of DNA bases by forming phosphodiesters. After cutting insert and plasmid with the same set of restriction enzymes both linear DNA fragments anneal by baseparing and formation of hydrogen bonds. Ligase activity connects the loosely bound pieces to a new circular recombinant plasmid.

For a ligation reaction, plasmid and insert were mixed in a ratio of 1:1 (plasmid : insert) combined with T4 DNA ligase (Boehringer) and T4 ligase buffer and incubated overnight at $16^{\circ} \mathrm{C}$. The success of the ligation was checked on an agarose gel. The ligated vector-insert product was either used directly for transformation of competent bacteria or stored at $-20^{\circ} \mathrm{C}$.

Standard ligation $(10 \mu 1)$ :

$1 \mu$ plasmid, $1 \mu 1$ insert, $1 \mu 1$ 10x T4 ligase buffer, $1 \mu 1$ T4 ligase, $6 \mu 1 \mathrm{H}_{2} \mathrm{O}$.

\section{II.2.7 $\quad$ Transformation of competent bacteria}

Genetic transformation of E. coli was performed as described (Hanahan, 1985). For in vivo amplification of vector DNA the $E$. coli XL1 blue strain was used while the strain BL21 was used for protein expression. The BL21 strain is deficient for certain proteases and therefore very suitable for expression of a foreign protein. 


\section{Materials and Methods}

Transformation was performed with the heat shock method. A $200 \mu 1$ aliquot of competent bacteria was thawed on ice and mixed with the ligation solution, i.e. astrin in the pET23a vector. After $30 \mathrm{~min}$ incubation cells were heat shocked at $42^{\circ} \mathrm{C}$ for exactly $100 \mathrm{sec}$ and subsequently cooled on ice. $800 \mu \mathrm{l}$ of complete medium (SOC) were added followed by $1 \mathrm{~h}$ incubation at $37^{\circ} \mathrm{C}$ on a shaker (recovery). To distinguish bacteria that were successfully transformed the cells were transferred to ampicillin containing solid agar. Only transformants could grow on this medium, because resistance against the antibiotic is located only on the transformed pET23a vector.

\section{II.2.8 Determination of nucleic acid concentration}

Two methods were applied to check the concentration of RNA or DNA. For estimation it was sufficient to compare the sample with a probe with known concentration on ethidium bromide stained agarose gels. For exact values the optical density was measured at a wavelength $260 \mathrm{~nm}$ and the concentration was calculated using the following formula:

$$
\begin{aligned}
& A_{200} \times 100=\quad \text { ODU (optical densty unts) } \\
& A_{200} \times 100 \times\left(\frac{33 \mathrm{ug}}{10 \mathrm{DU}}\right)=\quad \text { ugs (micrograms) } \\
& A_{200} \times 100 \times\left(\frac{33 \mathrm{ug}}{10 \mathrm{DU}}\right) \times\left(\frac{1 \mathrm{~g}}{1 \times 10^{6} \mathrm{ug}}\right) \times\left(\frac{1 \mathrm{~mole}}{\text { M.W. (in g) }}\right) \times\left(\frac{1 \text { micromole }}{1 \times 10^{-6} \text { moles }}\right)= \\
& A_{250} \times 100 \times\left(\frac{33 \mathrm{ug}}{10 \mathrm{DU}}\right) \times\left(\frac{1 \mathrm{~g}}{1 \times 10^{5} \mathrm{ug}}\right) \times\left(\frac{1 \mathrm{~mole}}{\text { M.W. (in g) }}\right) \times\left(\frac{1 \text { micromole }}{1 \times 10^{-6} \text { moles }}\right) \times\left(\frac{1}{0.001 \text { Liter }}\right)=
\end{aligned}
$$

(Source: http://www.dharmacon.com/tech/tech003.html) 


\section{II.3 Protein biochemical methods}

II.3.1 Discontinuous polyacrylamide gel electrophoresis

Molecular weight dependent separation of proteins was performed by discontinuous SDS-PAGE. SDS is a non-ionic detergent, which denatures the proteins and neutralises the charges. In the stacking gel (upper) the proteins are concentrated, in the separation gel (lower) separated (Weber \& Osborn 1969, Laemmli, 1970).

Gels with a SDS content of $0.1 \%(\mathrm{w} / \mathrm{v})$ and an acrylamide content of 7.5 or $10 \%(\mathrm{w} / \mathrm{v})$ in the separating gel were used. Polymerisation of the liquid gel solution was started by addition of the radical starter APS and TEMED, a catalysator. The separating gel was covered with 2-butanol and rinsed with water after polymerisation. Subsequently the stacking gel was added and the combs placed so as to form troughs in the stacking gel. Protein samples were mixed with $5 \mathrm{x}$ sample buffer (tso the concentration in the sample was $1 \mathrm{x}$ ) and boiled for 2-5 min (water bath). After recooling to room temperature they were loaded on the gel or stored at $-20^{\circ} \mathrm{C}$. Electrophoresis was carried out with $60 \mathrm{mV}$ in the stacking gel and $120 \mathrm{mV}$ in the resolving gel. After electrophoresis the resolving gel was either stained with Coomassie Brilliant Blue and subsequently photographed or was used for western blotting experiments (II.4.4.4).

Separating gel (lower):

$20 \mathrm{ml} \mathrm{H}_{2} 0,16 \mathrm{ml}$ acrylamide/bis solution, $12 \mathrm{ml}$ resolving gel buffer $(1.5 \mathrm{M}$ Tris-HCl, $\mathrm{pH}$ 8.8, $0.4 \%(\mathrm{w} / \mathrm{v})$ SDS), $150 \mu 1$ APS, $20 \mu 1$ TEMED

Stacking gel (upper):

$9 \mathrm{ml} \mathrm{H}_{2} \mathrm{O}, 2.25 \mathrm{ml}$ acrylamide/bis solution, $3.75 \mathrm{ml}$ stacking gel buffer $(0.5 \mathrm{M}$ Tris- $\mathrm{HCl}, \mathrm{pH}$ 6.8, $0.4 \%(\mathrm{w} / \mathrm{v})$ SDS), $150 \mu 1 \mathrm{APS}, 20 \mu 1$ TEMED

APS 10\% (w/v): 


\section{Materials and Methods}

Ammoniumperoxodisulfate in water (stored at $4^{\circ} \mathrm{C}$ in the dark)

Acrylamide/bis solution:

$30 \%(\mathrm{w} / \mathrm{v})$ acrylamide, $0.8 \%(\mathrm{w} / \mathrm{v}) \mathrm{N}, \mathrm{N}^{\prime}$-methylenbisacrylamide (ratio 37.5:1)

\section{II.3.1.1 Coomassie staining}

Separated proteins in the gels were visualised by staining with Coomassie Brilliant Blue (Roth, Munich). The gel was placed in the staining bath for $5 \mathrm{~min}$, rinsed with water and destained in destaining solution on a shaker overnight at room temperature.

Staining solution:

6.25 g Coomassie Brilliant Blue , $180 \mathrm{ml}$ acetic acid (96\%), $900 \mathrm{ml}$ methanol to $2000 \mathrm{ml}$ with water (final volume).

Destaining solution:

$1500 \mathrm{ml}$ methanol, $1000 \mathrm{ml}$ acetic acid (96\%) to $4000 \mathrm{ml}$ with water (final volume).

\section{II.3.2 Protein Expression}

Expression of a protein in a bacterial host requires the transcription of DNA to the corresponding mRNA and the subsequent translation into the primary amino acid structure at the ribosomes. The pET23a plasmid contains the strong $\mathrm{T} 7$ promoter, which acts as a binding site for the polymerase holoenzyme in the presence of IPTG (2-Isopropyl-ß-Dgalactopyranoside). IPTG causes gratuitous induction of the promoter and the cDNA of astrin is therefore permanently transcribed. For protein expression transformed bacteria were incubated overnight in $20 \mathrm{ml} \mathrm{LB}$ medium at $37^{\circ} \mathrm{C}$ as a preculture. The precultures were transferred to $800 \mathrm{ml}$ LB medium, which was subsequently incubated on a shaker 
$\left(37^{\circ} \mathrm{C}\right.$ ) until an optical density of $\sim 0.6$ (measured at a wavelength of $595 \mathrm{~nm}$ ) was observed and then induced with 10mM IPTG. After 4hrs incubation cells were harvested for protein purification.

\section{II.3.3 Purification of overexpressed proteins}

To purify the overexpressed recombinant protein, an inclusion body (IB) preparation was performed. Inclusion bodies are packages of aggregated, insoluble proteins, which often occur after overexpression of a foreign protein in a bacterial host. IBs can be purified relatively easy by centrifugation after application of non-ionic detergents such as Triton X-100 or Nonidet P-40 to lyse the cells (Georgiou et al., 1999).

Cells containing astrin (see protein expression) were harvested via centrifugation (6000rpm, $15 \mathrm{~min}, 4^{\circ} \mathrm{C}$ ). IB preparation was carried out at $4^{\circ} \mathrm{C}$ in the cold room or on ice. The pellet from the $800 \mathrm{ml}$ culture was homogenised with $25 \mathrm{ml}$ IB lysis buffer in a glass homogenizer and incubated on ice for $15 \mathrm{~min}$. Then $50 \mathrm{ml}$ of detergent buffer were added and the suspension was again homogenised. IBs were isolated by centrifugation (10000rpm, 25min, $4^{\circ} \mathrm{C}$ ) and washed by homogenisation in IB wash buffer and centrifugation as before. The wash step was repeated and after the second wash the pellet was resuspended in Mono-S buffer for subsequent FPLC (fast protein liquid chromatography). FPLC was done following the standard protocol for Mono-Q kation exchange columns. Protein elution was done with increasing $\mathrm{NaCl}$ concentration in the range $0-0.5 \mathrm{M}$. Protein concentrations of the eluted fractions were tested using the Bradford method as described (Bradford, 1976). 
IB lysis buffer (pH 8.0):

$50 \mathrm{mM}$ Tris, $1 \mathrm{mM}$ EDTA, 25\% (w/v) Sucrose, 0.5\% (w/v) lysozyme

Detergent buffer (pH7.5):

$20 \mathrm{mM}$ Tris, 2 mM EDTA, $0.2 \mathrm{M} \mathrm{NaCl}, 1 \%$ (w/v) DOC, 1\% (w/v) Nonidet P-40

IB wash buffer ( $\mathrm{pH} 7.5)$ :

$10 \mathrm{mM}$ Tris-HCl, $1 \mathrm{mM}$ EDTA, $0.5 \%(\mathrm{w} / \mathrm{v})$ Triton X-100

(Buffers were supplemented with protease inhibitor mix (Complete, Boehringer) prior to use.)

Mono-Q buffer (pH 6.6):

8.5 M Urea, 10 mM Phosphate buffer (see Maniatis “Molecular Cloning”, section B.21), 1 mM ß-mercaptoethanol

(elution buffer: additional $0.5 \mathrm{M} \mathrm{NaCl}$ )

\section{II.3.4 Purification of polyclonal antibodies}

Rabbits were immunised with either partial recombinant astrin proteins or synthetic peptides of 16 aa in length (see table) coupled to keyhole limpet hemocyanine (KLH) (commercial immunisation, BioScience, Göttingen). The synthetic peptides contained an N-terminal cysteine residue for subsequent sulfo-MBS coupling.

Two different procedures have been applied for antibody purification. Both were basically affinity chromatographies involving the antigen immobilised on a solid matrix.

For affinity chromatography with $\mathrm{BrCN}$ activated Sepharose 4B (Amersham, MA) the antigen peptide was linked to a sulfo-MBS-BSA construct. $25 \mathrm{mg}$ BSA were dissolved in $1 \mathrm{ml} \mathrm{PBS}$ and $9 \mathrm{mg}$ sulfo-MBS in $0.5 \mathrm{ml}$ PBS. Both fractions were mixed in one $2 \mathrm{ml}$ Eppendorf tube and rotated end over end for $30 \mathrm{~min}$ at room temperature. Unbound sulfo- 
MBS was removed by mixing the solution with $1 \mathrm{ml}$ PBS and applying it to a PD-10 column (Amersham). The column was equilibrated with $25 \mathrm{ml}$ sodium phosphate buffer. The sulfo-MBS/BSA solution was pipetted into the column and eluted with $4 \mathrm{ml}$ sodium phosphate buffer. Fractions of $0.5 \mathrm{ml}$ were collected and tested for protein by spotting $2 \mu \mathrm{l}$ of each on Whatman paper and then staining with Coomassie Brilliant Blue. To link the antigen to the sulfo-MBS construct $5 \mathrm{mg}$ of the antigen were dissolved in $1 \mathrm{ml}$ PBS and mixed with a positive fraction from the last coupling step and rotated end over end for three hours at room temperature. Unbound peptides were removed by dialysing the solution in a dialysis tube with a cut-off of $14 \mathrm{kDa}$ against sodium carbonate buffer overnight at $4^{\circ} \mathrm{C} .1 \mathrm{~g} \mathrm{BrCN}$ activated sepharose was dissolved in $5 \mathrm{ml} 1 \mathrm{mM} \mathrm{HCl}$ and activated with $250 \mathrm{ml} 1 \mathrm{mM} \mathrm{HCl}$ by stirring with a Pasteur pipette. After washing the swelling matrix with $250 \mathrm{ml}$ sodium carbonate buffer it was transferred to a Sarstedt tube and incubated with the sulfo-MBS/BSA-antigen construct overnight at $4{ }^{\circ} \mathrm{C}$. The matrix was sedimented by centrifugation for $1 \mathrm{~min}$ at $500 \mathrm{rpm}$ and unspecific binding was blocked with $10 \mathrm{ml} 1 \mathrm{mM}$ Tris $\mathrm{pH} 8$ for $1 \mathrm{~h}$ at RT. Antibodies were allowed to bind by incubating the matrix with $12 \mathrm{ml}$ serum overnight at $4^{\circ} \mathrm{C}$. The matrix was transferred to a disposable PVC column and washed three times with PBS. Low affinity antibodies were eluted with elution buffer pH8. After again washing the matrix with PBS the antibodies could be purified with $5 \mathrm{ml}$ elution $\mathrm{pH} 2.5$ buffer and were collected in $1.5 \mathrm{ml}$ tubes containing $200 \mu 1$ Tris-Base (pH 9.5) to neutralise the antibody. Subsequently the antibodies were immediately dialysed against PBS.

Alternatively $1.5 \mathrm{mg}$ of the purified antigen (recombinant protein) were subjected to SDS PAGE and the gel was Coomassie stained and electroblotted onto nitrocellulose (II.2.4.3). The band was cut from the nitrocellulose membrane and incubated in TBST 
containing $4 \%$ milk powder for $1 \mathrm{~h}$ at RT to block unspecific binding. Subsequently the membrane piece was cut in small pieces and incubated with $650 \mu \mathrm{l}$ serum overnight at $4^{\circ} \mathrm{C}$. After removing the supernatant the membrane pieces were washed with TBST, TBST containing $0.5 \mathrm{M} \mathrm{NaCl}$ and TBST $+0.5 \%(\mathrm{v} / \mathrm{v})$ Triton X-100. Antibodies were eluted by vortexing the membrane pieces with $550 \mu 1100 \mathrm{mM}$ glycine (pH 2.5) and the supernatant was mixed with $50 \mu 11 \mathrm{M}$ Tris-Base to neutralise the samples.

\section{II.4 siRNA technique}

II.4.1 $\quad$ Selection of siRNA sequences for targeting specific mRNAs

To design siRNA duplexes against a specific target mRNA it is necessary to know the correct sequence of at least 20 nucleotides of the targeted mRNA. Since recent studies show that the silencing (RNA interference) occurs within the cytoplasm any intron sequences should be neglected. Information on sequences of mature mRNA can be taken from EST or mRNA databases (e.g. www.ncbi.nih.gov, www.ebi.ac.uk or www.allgenes.org).

Therefore the target regions were selected from the open reading frame of the corresponding cDNA, preferably within the first few hundred nt downstream of the start codon. Search criterion was the sequence $5^{\prime}$-AA(N19)UU where $\mathrm{N}$ is any nucleotide. Candidate sequences had a $\mathrm{G} / \mathrm{C}$-content of 40 to $60 \%$. siRNA oligonucleotides were synthesised as $5^{\prime}-(\mathrm{N} 19) \mathrm{TT}$ for sense and $5^{\prime}-\left(\mathrm{N}^{\prime} 19\right) \mathrm{TT}\left(\mathrm{N}\right.$ and $\mathrm{N}^{\prime}$ indicate any ribonucleotide, $\mathrm{T}$ is $2^{\prime}$-deoxythymidine) for antisense orientation. To ensure that only one gene will be targeted the siRNA sequences were subjected to a Blast-search against human EST libraries and known mRNA sequences. 
These criteria led to a variety of siRNA sequences (Table 1) targeting the ORF of astrin mRNA (accession number A7399910). As siRNA control a sequence against firefly (Photinus pyralis) luciferase (accession number X65324) was used (pGL2 siRNA). As a positive control for knockdown a siRNA for the kinesin related motor protein $\mathrm{Eg} 5$ or for the nuclear envelope protein lamin A/C (Elbashir et al., 2001a) were used. Single stranded siRNA oligonucleotides were duplexed as described (Elbashir et al., 2001a) (if necessary) and used for transient transfection of human cell lines.

Table 1: siRNA sequences and targets

\begin{tabular}{|c|c|c|c|}
\hline Gene & Acc. No. & $\begin{array}{l}\text { siRNA } \\
\text { pos. }\end{array}$ & MRNA SEQUENCE \\
\hline $\begin{array}{l}\text { pGL2 (control) } \\
\text { EGFP } 1 \\
\text { EGFP2 } \\
\text { human (homo } \\
\text { sapiens) }\end{array}$ & X65324 & $153-175$ & $\begin{array}{c}\text { CACGUACGCGGAAUACUUCGAAA } \\
\text { AAGACCCGCGCCGAGGUGAAG } \\
\text { AAGAACGGCAUCAAGGUGAAC }\end{array}$ \\
\hline Lamin $\mathrm{A} / \mathrm{C}$ & NM_005572 & $608-630$ & AACUGGACUUCCAGAAGAACAUC \\
\hline Lamin A & $\mathrm{X} 03 \overline{4} 44$ & $\begin{array}{l}1919- \\
1941\end{array}$ & AAUCUGGUCACCCGCUCCUACCU \\
\hline Vimentin & NM_003380 & $346-368$ & AACUACAUCGACAAGGUGCGCUU \\
\hline Lamin B1 & NM_005573 & $672-694$ & AACGCGCUUGGUAGAGGUGGAUU \\
\hline Beta Actin & NM_001101 & $972-994$ & AAUGAAGAUCAAGAUCAUUGCUC \\
\hline Gamma Actin & NM_001614 & $8-30$ & AAGAGAUCGCCGCGCUGGUCAUU \\
\hline Eg5 & NM_004523 & $\begin{array}{l}1547- \\
1569\end{array}$ & AACUGGAUCGUAAGAAGGCAGUU \\
\hline CENPE & NM_001813 & $944-966$ & AAACUCUUACUGCUCUCCAGUUU \\
\hline cyt. Dynein & U535̄ 30 & $509-531$ & AAGGCCAAGGAGGCGCUGGAAUU \\
\hline Scc1 & D38551 & $\begin{array}{l}1216- \\
1238\end{array}$ & AAAGGAGGAGAGGCAGAUAAUUU \\
\hline Smc1 & NM_014865 & $\begin{array}{l}2603- \\
2625\end{array}$ & AAGAGGUGGCAGUGACCCUCAUU \\
\hline Smc3 & AF020043 & $\begin{array}{l}1132- \\
1154\end{array}$ & AAGCAGGGUCGAGGAAGCCAGUU \\
\hline Securin & NM_004219 & $303-325$ & AAGCUCUGUUCCUGCCUCAGAUG \\
\hline Separin & NM_012291 & $\begin{array}{l}1055- \\
1077\end{array}$ & AAGCACAGGCCUUGCUGUGGCUU \\
\hline INCENP & AY714053 & $766-788$ & AAGCUCAGGAUUGCGCAGGUCUC \\
\hline
\end{tabular}




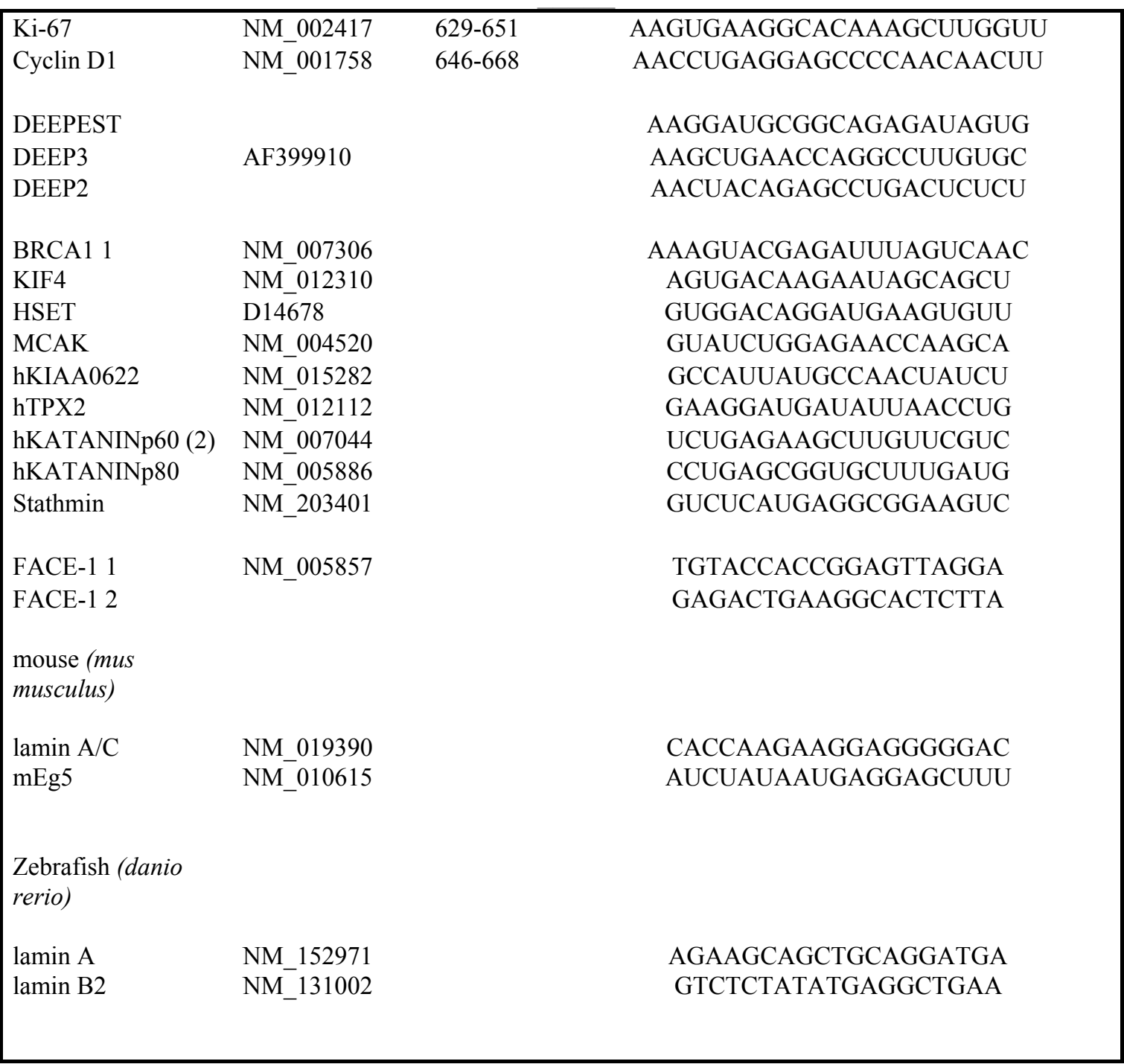

\section{II.4.2 Preparation of siRNA Duplexes}

The pGL2 siRNAs were provided in desalted and deprotected form, and stored in water at $-20^{\circ} \mathrm{C}$. Astrin specific siRNAs were delivered in either desalted and deprotected form (Dharmacon, option B, www.dharmacon.com/sirna) or as ready to use HPLC purified duplexes (option C) synthesised as (N19)dTdT.

Single stranded sense and antisense siRNAs in a final concentration of $20 \mu \mathrm{M}$ were combined with $70 \mu 12 \mathrm{x}$ annealing buffer and RNAse free (provided by supplier if siRNAs, Dharmacon) water was added to $140 \mu$ final volume. After denaturation of the 
RNA for $1 \mathrm{~min}$ at $95^{\circ} \mathrm{C}$ the solution was incubated for $1 \mathrm{~h}$ at $37^{\circ} \mathrm{C}$ on a shaker. siRNA duplexes can be stored at $-20^{\circ} \mathrm{C}$ and usually stay stable for several freeze thaw cycles. Usually the duplex solutions were aliquoted in portions of $30 \mu 1$ before freezing. This was to prevent contamination and degradation of the duplexes.

2x annealing buffer:

$\begin{array}{lll}\text { potassium acetate } & 200 \mathrm{mM} & \mathrm{pH} 7.4 \\ \text { Magnesium acetate } & 4 \mathrm{mM} \\ \text { HEPES-KOH } & 60 \mathrm{mM} & \end{array}$

II.4.3 Transient transfection of human cell lines with siRNA duplexes

The experimental procedure for siRNA induced gene silencing was basically performed as described (Elbashir et al., 2001a). The following paragraphs give a detailed collection of the protocols used from preparation of the cells to the final detection methods. Some parts would also fit in other sections of this methods part, but since the novel technique of siRNA mediated gene silencing is an essential part of the work the related procedures are put together for more convenience and better understanding.

\section{II.4.3.1 $\quad$ Preparation of cells}

Cells were split one day prior to the transfection. Medium was discarded from one Petri dish with $9.5 \mathrm{~cm}$ diameter containing an 80 to $90 \%$ confluent cell layer (HeLa SS6 or MCF-7). The dish was washed once with trypsin-EDTA solution (Life Technologies, \#25300-054, used in a 1:100 dilution) prewarmed to $37^{\circ} \mathrm{C}$ or PBS and subsequently trypsinized with $1 \mathrm{ml}$ trypsin-EDTA for $2 \mathrm{~min}$ at $37^{\circ} \mathrm{C}$. The cells were resuspended with a $5 \mathrm{ml}$ glass pipette and transferred into antibiotic free DMEM containing 10\% FCS (10 drops of the cell solution from $5 \mathrm{ml}$ pipette into $40 \mathrm{ml}$ of medium). The cell solution was 
transferred to 24 well plates $(1.5 \mathrm{~cm}$ diameter per well, Nunc, Wiesbaden) $(532 \mu 1$ per well). If silencing of genes was to be assayed by TUNEL test (II.4.5 ) or immunofluorescence microscopy (II.4.4.2) glass coverslips of $1.3 \mathrm{~cm}$ diameter were put on the bottom of the well before adding the cells. For flow cytometry (see II.4.6) cells were grown in 6-well plates ( $3 \mathrm{~cm}$ diameter per well) (Nunc) in $2 \mathrm{ml}$ medium (cell suspension as described above).

\section{II.4.3.2 Transfection of siRNA duplexes}

Most of the transfections used in this study based on the standard protocol using cationic liposomes (Felgner and Ringold, 1989, Elbashir et al., 2001) (see II.4.3.3).

\section{II.4.3.3 Transfection with cationic liposomes}

Transfection was performed not later than 24 hours after plating the cells when a confluence of 40 to $60 \%$ was reached $\left(3 \times 10^{4}\right.$ to $1 \times 10^{5}$ cells per well in 24 well plate, depending on cell line and growth rate). I used two different transfection reagents Oigofectamine (Invitrogene, Groningen, Netherlands) and TransIT-TKO: siRNA Transfection Reagent (Mirus Corporation, Madison, Wisconsin). Both perform exogenous delivery of siRNA into cells by forming liposome/nucleic acid complexes which are taken up by the cell and the nucleic acid duplexes are subsequently released within the cytoplasm (Felgner et al., 1989).

Two premixes were prepared for each transfection reagent. Given quantities were used for one well of the prepared 24 well plates (for one well in 6 well plate quantities were multiplied by four). For Oligofectamine transfection premix 1 contained $3 \mu 120 \mathrm{mM}$ (60 pmol) siRNA duplexes and $50 \mu 1$ Opti-Mem 1 (serum free medium, Life 
Technologies). Premix 2 consisted of $4 \mu \overline{1}$ Oligofectamine and $12 \mu 1$ Opti-Mem 1. After incubation for 5-8 min at room temperature the premixes were combined and incubated for an additional 15-25 min for complex formation until the solution turned turbid. The liposome complexes were added to the cells without replacing the growth medium and gently mixed by rocking the plate by hand.

The TranIT TKO transfection reagent (Mirus, Davos) was handled in the same way, except that the premixes had the following compositions: premix 1 contained $3 \mu 1$ $20 \mathrm{mM}$ siRNA duplexes and $12 \mu \mathrm{l}$ Opti-Mem, premix 2 contained $4 \mu 1$ Mirus reagent and $50 \mu 1$ Opti-Mem, so that basically the transfection reagent was preincubated in the larger volume.

Transfected cells were incubated at $37^{\circ} \mathrm{C}$ in a $5 \% \mathrm{CO}_{2}$ atmosphere in the incubator.

\section{II.4.3.4 Microinjection of siRNAs}

For microinjection of siRNAs cells were seeded in their standard culture medium on labelled coverslips (CellLocate, Eppendorf, Hamburg) 24 hours prior to injection. A 6 $\mu \mathrm{M}$ solution of siRNAs in $1 \mathrm{x}$ annealing buffer was injected into the cytoplasm of cells with an Eppemdorf Micromanipulator.

\section{II.4.3.5 Pinocytosis mediated transfection of siRNAs}

$24-36$ hours prior to treatment cells were trypsinized and diluted 1:5 with fresh medium without antibiotics $\left(1-3 \times 10^{5}\right.$ cells $\left./ \mathrm{ml}\right)$ and transferred to 24 well plates $(500 \mu 1$ per well). Cells were $40-60 \%$ confluent when the pinocytosis procedure was performed. Hypertonic medium was prepared using a sucrose/PEG mixture used according to the manufacturer's directions [Influx Pinocytic Cell-Loading Reagent (I-14402), Molecular 


\section{Materials and Methods}

Probes, Eugene, OR] or alternatively see (Okada and Rechsteiner, 1982). The sucrose/PEG mixture was preheated to $80^{\circ} \mathrm{C}$ for 10 minutes to melt the PEG. Then $4.7 \mathrm{ml}$ of prewarmed $\left(37^{\circ} \mathrm{C}\right)$ serum free DMEM, $250 \mu 1$ of fetal calf serum and $50 \mu 1$ of $1 \mathrm{M}$ HEPES in phosphate buffered saline, $\mathrm{pH} 7.4$, were added to the sucrose/PEG mixture to form the hypertonic medium. The hypotonic medium was prepared by diluting serum free DMEM with sterile deionized water in a 6:4 ratio. siRNAs targeting either Eg5, LMN A/C or Ki67, were diluted in hypertonic medium prewarmed to $37^{\circ} \mathrm{C}$ to a final concentration of $0.4 \mu \mathrm{M}$. The initial culture medium in each well was discarded and replaced by $200 \mu \mathrm{l}$ of hypertonic medium containing the siRNAs for loading of the cells. After 10 or 15 minutes at $37{ }^{\circ} \mathrm{C}$ the hypertonic medium was replaced with hypotonic medium prewarmed to $37{ }^{\circ} \mathrm{C}$, equilibrated with $5 \% \mathrm{CO}_{2}$ and incubated for 2 minutes at room temperature. Incubation with the hypotonic medium caused an osmotic shock that led to lysis of the pinosomes and release of the siRNAs into the cytoplasm. After 2 minutes the hypotonic medium was replaced by $1 \mathrm{ml}$ of standard DMEM medium prewarmed to $37^{\circ} \mathrm{C}$ to allow recovery of the cells. Cells were incubated at $37{ }^{\circ} \mathrm{C}$ with $5 \% \mathrm{CO}_{2}$ until analysis.

When a double transfection procedure was used the whole procedure was repeated usually after a 10 hour interval.

Control cells were subjected to the osmotic lysis of pinosomes procedure without the addition of siRNAs to the hypertonic medium. 

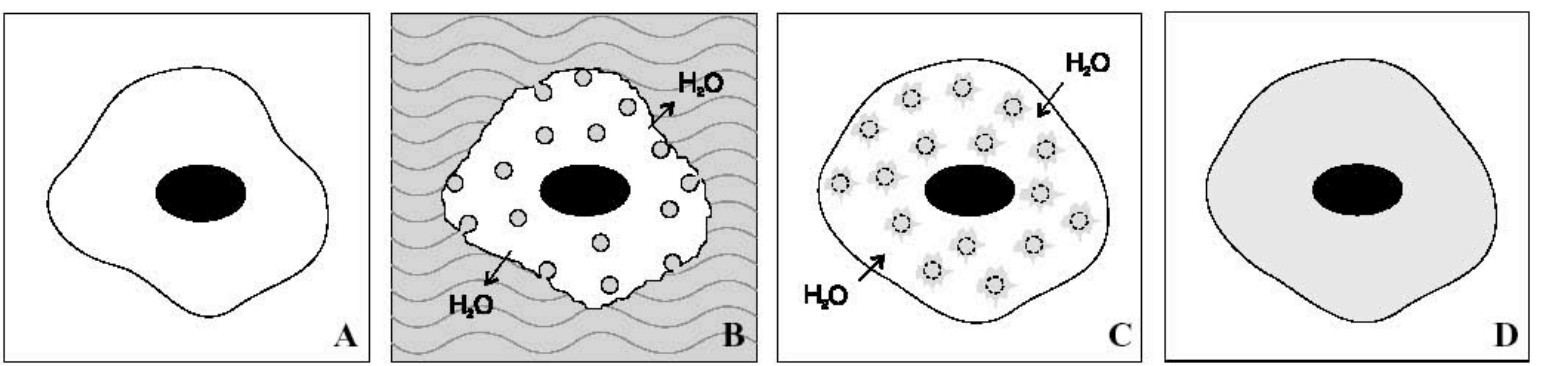

Figure MI: The principle of pinocytosis mediated transfection. (A) shows a cell in standard culture medium. In (B) the cell is transferred into hypertonic medium containing siRNAs. The cell starts pinocytotic uptake of loading medium. When transferred into hypotonic medium (C) water flows into the cell and pinocytotic vesicles. The vesicles burst and release the carg (siRNAs) into the cytoplasm. The cell is transferred into standard culture medium for recovery (D). (Modified from Influx pinocytic cell loading reagent handbook, Molecular Probes)

\section{II.4.3.6 Transfection by electroporation}

Human HL60 cells were transfected with siRNAs by the Amaxa nucleofector technology (Amaxa, Cologne) which is basically optimized electroporation. It consists of the Nucleofector device, which delivers the electrical parameters, and Nucleofector Solutions, in which the cells are contained while the electrical program is executed. For each cell type, optimized conditions can be developed. A solution of $5 \mu \mathrm{M}$ siRNA in manufacturer's buffer D was used with the optimized electroporation program for HL60 cells. Performance of electroporation was according to the manufacturer's protocol.

\section{II.4.4 Detection of siRNA mediated gene silencing}

Various methods can be used to detect the phenotype of knockdown cells. The easiest assay is monitoring the morphological changes by phase contrast microscopy (II.4.4.1). Presence or absence of the protein can be checked by indirect immunofluorescence microscopy (II.4.4.2) or by immunoblotting (II.4.4.4) if a target specific antibody against the silenced gene product is available. Essential genes have 


\section{Materials and Methods}

lethal effects once cells are lacking them, e.g. after silencing. In situ cell death can be shown by apoptosis detection procedures, e.g. the TUNEL test (II.4.4.5) or flow cytometrical measurements of the DNA content in the cell (II.4.4.6). The latter method can also be used to determine the cell cycle distribution of a cell population.

\section{II.4.4.1 $\quad$ Phase contrast microscopy}

To check for morphological changes siRNA treated cells were grown on coverslips with $0.8 \mathrm{~cm}$ diameter. For examination cells were mounted in HEPES-buffered DMEM containing $10 \%$ FCS and observed using a Plan-Neofluar $25 \mathrm{x} / 0.8$ objective. Apoptotic cells show micronuclei and blebbing of the cell membrane while cells arrested in mitotis round up and show condensed chromosomes.

\section{II.4.4.2 Indirect immunofluorescence microscopy}

Detection of gene knockdown could be monitored by immunofluorescence microscopy. The first step was the fixation of siRNA treated cells. A standard methanol fixation, which preserves some aspects of cell morphology and permeabilizes the cell for antibody penetration was suitable for our purpose.

For detection of target knockdown, cells were assayed 22, 40 and 60 hours post transfection. Coverslips with cells were incubated in ice cold methanol for 6 min. Excess methanol was removed from coverslips by touching them to dry filter paper. After transferring the air dried coverslips to a wet chamber the first antibody was applied. We used $30 \mu \mathrm{l}$ of antibody solution per coverslip and allowed target binding for $1 \mathrm{~h}$ at $37^{\circ} \mathrm{C}$. After incubation with the first antibodies the coverslips were washed in PBS as before and transferred into the wet chamber. An appropriate concentration of fluorescently labelled 


\section{Materials and Methods}

secondary antibodies against the immunoglobulin type of the corresponding first antibody [FITC conjugated goat anti-mouse IgG or rhodamine goat anti-rabbit IgG (Dianova, Hamburg)] was applied as above and incubated for 45 to $60 \mathrm{~min}$ at $37^{\circ} \mathrm{C}$. Subsequently the coverslips were washed with PBS and a Hoechst stain was applied to visualise the cellular chromatin. For a Hoechst stain $40 \mu 1$ of dye solution $(1 \mu \mathrm{M}$ Hoechst 33342, Hoechst, Frankfur/M) were put on each coverslip and incubated for 4 min at room temperature. The coverslips were then rinsed with PBS, excess liquid was soaked off with filter paper and the coverslips were mounted on microscope slides with Mowiol mounting medium (Hoechst, Frankfurt/M) and secured with nail polish. Cells were examined on a Zeiss Axiophot with a F Fluar 40x/1.30 oil objective and Metamorph imaging software (Universal Imaging Corporation, PA). Identical exposure times have been used for silenced and control cells.

Mowiol mounting medium: $2.4 \mathrm{~g}$ Mowiol 4-88 (Hoechst), $6 \mathrm{~g}$ glycerine, 12

(Osborn et al., 1998) $\quad \mathrm{ml} 0.2 \mathrm{M}$ Tris- $\mathrm{HCl} \mathrm{pH} 8.5,6 \mathrm{ml} \mathrm{H} 2 \mathrm{O}$

(Compounds were mixed and incubated at $50^{\circ} \mathrm{C}$ for $1 \mathrm{~h}$ with occasional shaking until the Mowiol was dissolved. To clarify the solution it was centrifuged for $5 \mathrm{~min}$ at $5000 \mathrm{rpm}$.

\section{II.4.4.3 Microscopic growth rate determination (CellScreen)}

The CellScreen system (innovatis, Bielefeld) allowed automated cell counting of undyed live adherent cultures, without sampling. Since the optical analysis process does not adversely affect the analysed cell culture, multiple measurements of the same culture 
were possible. Cellscreen counts the number of cells per square millimetre culture area when automatically analysing cell cultures. Cells were seeded in 24 well plates and subjected to repeated measurements. Measurements resulted in growth rate curves representing the cell covered area of the well surfaces.

\section{II.4.4.4 Immunoblotting}

In addition to fluorescence microscopy a protein knockdown was monitored by immunoblotting of extracts from knockdown and control cells. Cell extracts were made according to the following procedure:

The culture medium was removed from knockdown or control cell cultivated in 24 well plates. After rinsing the cells with $200 \mu 1$ PBS they were trypsinized with trypsinEDTA (as in II.4.3.1) for $1 \mathrm{~min}$ at $37^{\circ} \mathrm{C} .800 \mu 1$ DMEM containing 10\% FCS was added to quench the trypsin, and the cells were resuspended and transferred to chilled Eppendorf tubes. Cells were collected by centrifugation at $4000 \mathrm{rpm}$ for $5 \mathrm{~min}$ at $4{ }^{\circ} \mathrm{C}$ and resuspended in ice cold PBS. After an additional centrifugation step as before the supernatant was discarded and cells from one well were taken up in $25 \mu 1$ of pre-heated $\left(90^{\circ} \mathrm{C}\right) 2 \mathrm{x}$ Laemmli SDS sample buffer (BioRad, Munich) and incubated in boiling water for $5 \mathrm{~min}$ and vortexed. These cell extracts were applied to SDS PAGE (see II.3.1). Proteins from SDS PAGE minigels were transferred onto nitro-cellulose membranes using the 'tank-blot' method (Towbin et al., 1979). The gel was placed on a nitro-cellulose membrane of equal size and sandwiched between 3 layers of Whatman papers on each side. This stack was introduced into the blot chamber (BioRad Trans-Blot Cells, BioRad) with the nitro-cellulose membrane pointing towards the plus pole and electroblotted at 335 $\mathrm{mA}$ for $60 \mathrm{~min}$ at $4^{\circ} \mathrm{C}$ (cold room). Ponceau S (Sigma, Munich) staining of the nitro- 
cellulose membrane verified successful protein transfer. Incubating the membrane in TBST containing 5\% (w/v) milk powder (Skim Milk, Sigma) for $1 \mathrm{~h}$ at $37^{\circ} \mathrm{C}$ blocked unspecific protein binding.

The blocking solution was removed and fresh blocking solution containing the primary antibody at an appropriate dilution (Table 2) was added. After washing the blot 4 times with TBST for $10 \mathrm{~m}$ in the second antibodies [HRP conjugated swine anti-mouse or HRP conjugated goat anti-rabbit immunglobulins (Dako, Denmark)] were applied diluted in blocking buffer. After $45 \mathrm{~min}$ incubation at $37^{\circ} \mathrm{C}$ the blots were washed as before and the ECL detection was performed according to the protocol given by the manufacturer (Amersham Pharmacia).

Table 2: Dilutions and sources of antibodies

\begin{tabular}{|c|c|c|c|c|c|}
\hline $\begin{array}{l}\text { Antibody } \\
\text { (against) }\end{array}$ & Source Label & $\begin{array}{l}\text { clonel } \\
\text { sequencel } \\
\text { batch }\end{array}$ & IF dilution & WB dilution & Remarks \\
\hline$\square$ tubulin & $\mathrm{M}$ & DM1A & $1+750$ & & SIGMA \\
\hline Deep1 & $\mathrm{R}$ & R699 & $1+3$ & $1+1$ & Polyclonal ab, affinity \\
\hline Ast715 & $\mathrm{R}$ & p715-732 & $1+30$ & $1+50$ & purified \\
\hline Ast699 & $\mathrm{R}$ & p699-717 & $1+25$ & $1+15$ & \\
\hline vimentin & $\mathrm{M}$ & V9 & $1+2000$ & $1+1500$ & (Osborn et al, 1984) \\
\hline$\square$ tubulin & $\mathrm{M}$ & & $1+1000$ & & Sigma \\
\hline FACE-1a & $\mathrm{R}$ & & $1+500$ & & Polyclonal ab, affinity \\
\hline FACE-1b & $\mathrm{R}$ & & $1+400$ & & purified \\
\hline prelamin A & G & F0130 & $1+150$ & & SantaCruz \\
\hline prelamin A & $\mathrm{R}$ & & $1+500$ & & $\begin{array}{l}\text { Polyclonal ab, affinity } \\
\text { purified }\end{array}$ \\
\hline $\operatorname{lamin} \mathrm{A} / \mathrm{C}$ & $\mathrm{M}$ & 636.23 & $1+500$ & $1+50$ & (M. Osborn) \\
\hline $\operatorname{lamin} \mathrm{A}$ & $\mathrm{M}$ & JOL4 & $1+200$ & & abcam \\
\hline lamin B1 & $\mathrm{M}$ & & $1+250$ & & Zytomed \\
\hline lap2 & $\mathrm{M}$ & clone27 & $1+1000$ & & Transduction labs \\
\hline emerin & M & $4 \mathrm{G} 5$ & $1+250$ & $1+100$ & Novocastra \\
\hline GFP & $\mathrm{R}$ & & & & Sigma \\
\hline Ki67 antigen & $\mathrm{M}$ & Ki67 & $1+120$ & & Novocastra \\
\hline
\end{tabular}




\begin{tabular}{|c|c|c|c|c|c|c|}
\hline mouse lamin A & & & 346 & $1+5$ & $1+10$ & \\
\hline danio lamin A & & & & $1+150$ & & Georg Krohne \\
\hline danio lamin B2 & & & & $1+100$ & & Georg Krohne \\
\hline goat IgG & & & & $1+150$ & & Dianova \\
\hline mouse IgG & & & & $1+80$ & & Dianova \\
\hline mouse IgG & Goat & FITC & $\mathrm{mIgG}$ & $1+40$ & & Dianova \\
\hline rabbit IgG & & & & $1+80$ & & Dianova \\
\hline rabbit IgG & Goat & Rhd & rIgG & $1+40$ & & Dianova \\
\hline goat IgG & swine & HRP & & & $1+5000$ & Dako \\
\hline rabbit IgG & Goat & HRP & rIgG & & $1+10000$ & Dako \\
\hline mouse IgG & Swine & HRP & $\mathrm{mIgG}$ & & $1+10000$ & Dako \\
\hline
\end{tabular}

$(\mathrm{m}=$ murine monoclonal, $\mathrm{r}=$ rabbit polyclonal, FITC $=$ fluorescein, $\mathrm{HRP}=$ horse radish peroxidase, $\mathrm{IF}=$ immunofluorescence, $\mathrm{WB}=$ western blot, $\mathrm{Rhd}=$ rhodamine, $\mathrm{mIgG}=$ mouse immunglobulin $\mathrm{G}, \mathrm{rIgG}=$ rabbit immunglobulin $\mathrm{G})$

To compare loading of proteins the antibodies were stripped from the blots with a kit (Re-Blot Western Blot Recycling Kit, Chemicon) and the blots were reprobed with antibodies against a highly abundant protein (in this case vimentin).

\section{II.4.4.5 TUNEL test for apoptosis detection}

Apoptosis indicating DNA fragmentation can be monitored by a TUNEL test. In this assay free $3^{\prime}-\mathrm{OH}$ ends of fragmented DNA are labelled with FITC tagged deoxynucleotidetriphosphates by the enzyme terminal deoxynucleotidyl thiolase (TdT) which is component of the In situ Cell Death detection Kit (Roche). The assay was basically according to the manufacturers handbook. Cells on coverslips were methanol fixed, washed with PBS and permeabilised with permeabilisation buffer for 2 min on ice. After washing the coverslip 3 times with PBS excessive liquid was removed with a filter paper and the staining solution containing TdT and labelled nucleotides was applied (30 $\mu 1$ per coverslip). After $1 \mathrm{~h}$ incubation at $37^{\circ} \mathrm{C}$ in a wet chamber the coverslips were washed 4 times with PBS and were then examined by fluorescence microscopy. 
Permeabilisation buffer: $\quad$ PBS containing $\quad \mathrm{pH} 7.4$

Sodium citrate $0.1 \%(\mathrm{w} / \mathrm{v})$

Triton X-100 $0.1 \%(\mathrm{v} / \mathrm{v})$

For FACS analysis (II.4.4.6) cells in suspension were treated with TUNEL reagents.

II.4.4.6 Flow cytometry measurements to detect cell cycle Distribution, cellular DNA contents and apoptosis

For flow cytometrical measurements $\sim 0.8$ to $1.2 \times 10^{6}$ cells (adjusted using a "Neubauer" counting chamber) were grown in petri dishes for 48,66 or 74 h post transfection. After removing the culture medium cells were rinsed with prewarmed PBS $\left(37^{\circ} \mathrm{C}\right)$ and resuspended using $100 \mathrm{mM}$ EDTA in PBS. For fixation the cells were injected into ice cold 99.9\% ethanol using a disposable insulin injector (size: $100 \mu 1$ ) (Roche) and stored at $-20^{\circ} \mathrm{C}$. Cells could be assayed $2 \mathrm{~h}$ after fixation or alternatively be stored at $-20^{\circ} \mathrm{C}$ for several days. To determine the DNA content of the cells a propidium iodide stain was performed. The cells were collected by centrifugation at $2000 \mathrm{rpm}$ for $5 \mathrm{~min}$, the ethanol was discarded and subsequently the cells were transferred to $10 \mathrm{ml} \mathrm{FACS}$ analysis tubes. The cells were washed twice with $6 \mathrm{ml}$ ice cold PBS and centrifuged for $5 \mathrm{~min}$ at $2000 \mathrm{rpm}$. For permeabilisation cells were incubated in $0.2 \%$ Triton X-100 in PBS at room temperature. After washing twice with PBS the cells were collected by centrifugation ( $5 \mathrm{~min}, 2000 \mathrm{rpm}$ ) and incubated in $80 \mu \mathrm{T}$ TUNEL staining solution (see II.4.4.5) for $1 \mathrm{~h}$ at $37^{\circ} \mathrm{C}$. Cells were then washed in PBS ( 3 times in $8 \mathrm{ml}$ ), centrifuged as before and incubated in $500 \mu \mathrm{l}$ of the propidium iodide staining solution for $1 \mathrm{~h}$ at $37^{\circ} \mathrm{C}$. The staining solution contained RNAse A (Boehringer, Mannheim) to eliminate the 
background of dsRNA. Granulation, size and fluorescence in propidium iodide and fluorescein channels were examined with a FACScan (BD Bioscience) flow cytometer and the appropriate FACScan software for Macintosh computers. A total number of 10,000 events was counted and measured per sample.

Staining solution: $\quad$ PBS

pH 7.3

$\begin{array}{ll}\text { Propidium iodide } & 0.5 \mathrm{mM} \\ \text { RNAse A } & 10 \mu \mathrm{g} / \mathrm{ml}\end{array}$

II.4.4.7 Determination of total cell numbers using the Cell Counter and Analysis System $\left(\mathrm{CASY}^{\circledR}\right)$

The examination of growth rates after transfection and the detection of dead cells in the cell suspension was carried out using the Cell Counter and Analysis System (CASY ${ }^{\circledR}$ Model TT, Schärfe System, Munich). Function of this apparatus is two fold: I) it is based on pulse field analysis for signal measurement which works with digital pulse processing (DPP) and digitally records electric signals. Ii) the electric resistance is measured within the flow capillary allowing advanced particle measurement.

Cells were resuspended in a special isotonic solution (CASY ${ }^{\circledR}$ ton, Schärfe System) and passed the capillary (100 $\mu \mathrm{m}$ inner diameter) in a constant flow. Platinum electrodes applied a $1 \mathrm{MHz}$ low current field to the capillary. Living cells passing the capillary have a characteristic electric resistance, which allows the cell volume to be measure after automated integration of the signal strength. The plasma membranes of dead cells no longer have an electrical barrier and therefore these cells usually appear to have the size of the nucleus only. Apoptotic cells have a decreasing volume, which can also be measured. 
For CASY measurements medium from one well of a 6 well plate containing siRNA transfected cells was discarded and the cells were trypsinized with $0.25 \mathrm{ml}$ trypsinEDTA solution (II.4.2). After $1 \mathrm{~min}$ incubation at $37^{\circ} \mathrm{C}$ the trypsin was quenched by adding $1.5 \mathrm{ml}$ DMEM $+10 \%$ FCS. $100 \mu 1$ of the solution were mixed with $10 \mathrm{ml}$ of $\mathrm{CASY}^{\circledR}$ ton and directly used for cell counting. If cell numbers were too high the solution was diluted further with $\mathrm{CASY}^{\circledR}$ ton.

\section{II.4.4.8 The branched DNA assay for mRNA quantitation}

The branched DNA assay (QuantiGene Discovery System, Genospectra, Fremont, CA) was used for quantification of mRNA levels in cellular extracts as previously applied to quantitate insulin receptor mRNA (Wang et al., 1997). It is a very direct method for evaluation of mRNA levels, which requires neither RNA isolation and purification nor any amplification steps. The assay is based on specific cohybridization of the target mRNA and predesigned oligonucleotides. A probe set consists of three types of desoxyoligonucleotides: the capture extenders (CEs) are complementary to the target mRNA and to nucleotides which are immobilized in the capture plate, label extenders (LEs) also bind the target mRNA and the bDNA amplifier for subsequent enzymatic development and the blocking oligonucleotides (BLs). CEs, LEs and BLs are designed in a way to hybridize to a 300 to 500 nucleotide region along the target mRNA forming a DNA/RNA duplex.

The bDNA assay was performed according to the manufacturer's protocol. HeLa SS6 cells were grown in 96 well plates and transfected as described (all volumes where scaled down to $1 / 6$ when used for 96 well plate) and disrupted by application of lysis buffer containing CEs, LEs and BLs. $50 \mu \mathrm{l}$ of lysis buffer and $100 \mu 1$ medium were applied and cells were incubated for 1 hour at $37^{\circ} \mathrm{C}$. Cells lysates where transferred to the 
capture plates, plates were sealed and incubated at $52^{\circ} \mathrm{C}$ overnight. Capture plates were washed and incubated with an labelling/amplifier solution for 1 hour at $37^{\circ} \mathrm{C}$. Subsequently plates were developed for 30 minutes at $52^{\circ} \mathrm{C}$ with the substrate solution. Emitted luminescence was measured with a luminometer (VICTOR Light, Perkin Elmer, Wellesley, MA). Target mRNA specific probes for lamin A and FACE1 were used as well as a probe set for $\square$-actin mRNA. In the data shown in Fig 4B cell extracts were harvested 24 to 96 hours post transfection and developed with FACE1 or lamin A specific probes. Data represent levels of FACE1 mRNA (black) and lamin A mRNA (white) standardised with b-actin and normalised to values obtained with control cultures transfected with GL2 siRNA. FACE1 and lamin A mRNA levels are shown for FACE1 and lamin A silenced cells respectively. These values are expressed as a percentage of the value obtained after transfection with GL2 siRNA. 


\section{RESULTS}

\section{III.1 Human Targets I: RNAi against the human endoprotease FACE1}

III.1.1 The endoprotease FACE1 is required for the processing of prelamin A in $\underline{\text { HeLa cells }}$

Antibodies that detect specifically prelamin A, but not the processed lamin A, must recognize the C-terminal sequence of residues 647 to 664 (see Introduction). We have used two such antibodies: a commercial goat prelamin A antibody detecting a 20 residue sequence and a rabbit antibody which we raised with a synthetic peptide corresponding to residues 647 to 661 and purified by antigen affinity chromatography. Both antibodies gave the same results.

In normal HeLa cells, as well as in cells transfected with GL2 siRNA (Fig. 4A, top), the low level of prelamin $\mathrm{A}$ is detected as multiple small dots in the nucleoplasm and no staining of the nuclear lamina is observed. In contrast in cells that have been treated with FACE1 siRNA and then stained at different times after transfection (Fig. 4A, middle) the prelamin A accumulated with time at the nuclear lamina. Similar results showing effective silencing of FACE1 were also obtained on human MCF7 cells.

A strong accumulation of prelamin A was also detected in western blots of extracts from FACE1 silenced cells (Fig. 4B). A specific signal with the appropriate molecular weight for prelamin A was detected only in extracts from the silenced cells, but not in extracts from cells that were transfected either with the control GL2 siRNA targeting firefly (Photinus pyralis) luciferase or with lamin A siRNA.

Successful silencing of FACE1 by transfection of FACE1 siRNA was confirmed by the novel branched DNA (bDNA) assay (Fig. 4C). This assay allowed direct luminometric measurement of mRNA levels from cell extracts by specific 
immobilization of target mRNAs and labelling with DNA probes. Samples were derived from FACE1, LMNA or GL2 siRNA transfected HeLa cells. mRNA levels from GL2 cells were used for normalization of values obtained from silenced cell populations. The bDNA assay clearly showed reduction of about $80 \%$ in the FACE1 and LMNA mRNA levels already 24 hours after transfection. Thus, this new method appeared to be an appropriate technique for quantification of mRNA knockdown in RNAi experiments.

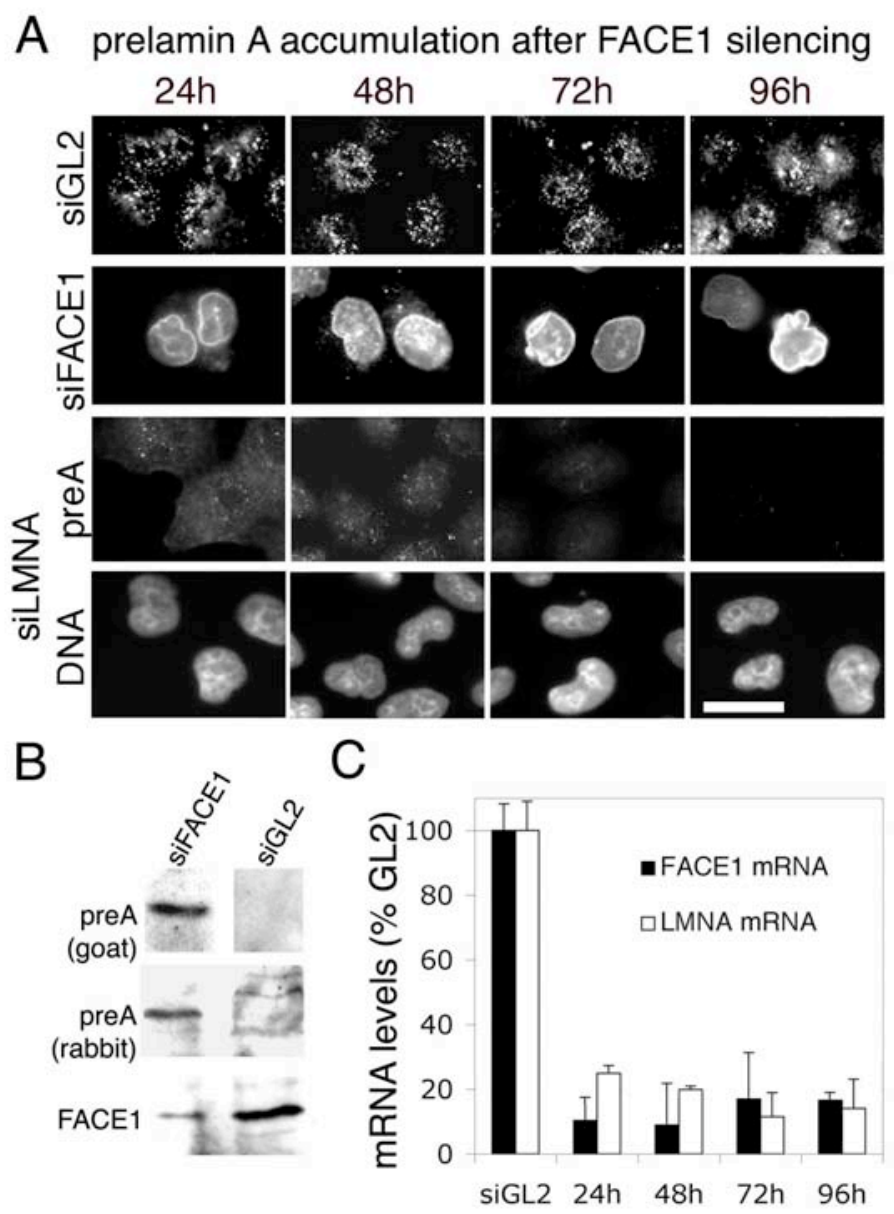

Figure 4: Silencing of human FACE1 results in accumulation of prelamin A.

Human HeLa SS6 cells were stained with the antibody SC06214 recognising an epitope in the carboxyterminal region of unprocessed lamin A. This antibody stains prelamin A, but not lamin A. (Row 1) Transfection with an unspecific GL2 siRNA did not affect the normal localisation of prelamin A in subnuclear foci. (Row 2) Transfection with FACE1 siRNA results in progressive accumulation of unprocessed prelamin A in the nuclear lamina. (Rows 3 and 4) Transfection of cells with lamin A 
siRNA targeting lamin A/C mRNA resulted in loss of prelamin A (Row 3). Hoechst stain of the same cells (Row 4). (B) Accumulation of prelamin A was confirmed by western blots of FACE1 silenced cells and control populations. Prelamin A accumulated in FACE1 silenced cells 48 hours after transfection, but was not detectable in extracts from cells transfected with the GL2 siRNA (upper panel goat FACE1 antibody, middle panel rabbit FACE1 antibody). (C) Silencing of FACE1 and lamin $\mathrm{A}$ in single siRNA experiments was confirmed by luminometric measurement of absolute mRNA levels via the branched DNA assay. Note that after 24 hours the mRNA is very much reduced for both targets and that these low levels are maintained at least until 96 hours after transfection. (Bar: $20 \mu \mathrm{m}$.)

The combined results suggest that after loss of the endoprotease FACE1 cells were unable to process farnesylated prelamin A by cleavage of the C-terminal 18 amino acids, and that in consequence prelamin $\mathrm{A}$ is incorporated in place of the normal, processed lamin A into the nuclear lamina.

\section{III.1.2 FACE1 knockdown causes changes in nuclear morphology}

Two populations could be distinguished in cells treated with FACE1 siRNA. The first population (Fig. 5) showed changes in nuclear morphology with many cells displaying micronuclei. The second population (Fig. 6) was formed by cells that arrested in aberrant mitosis and later underwent apoptosis.

Accumulation of prelamin A in HeLa cells led to dramatic changes in the nuclear architecture with increasing time after transfection with FACE1 siRNA (Fig. 5). These changes included folds in the nuclear envelope, herniations and crevices extending into the nuclear periphery as well as lobulation and increasing fragmentation of nuclei and formation of micronuclei. All effects could be detected already early after siRNA transfection ( 24 hours), but the percentage of cells displaying more severe effects increased with the time post transfection (see Fig. 5B). 120 hours post transfection $30 \%$ of the cells had micronuclei (Fig. 5C). 

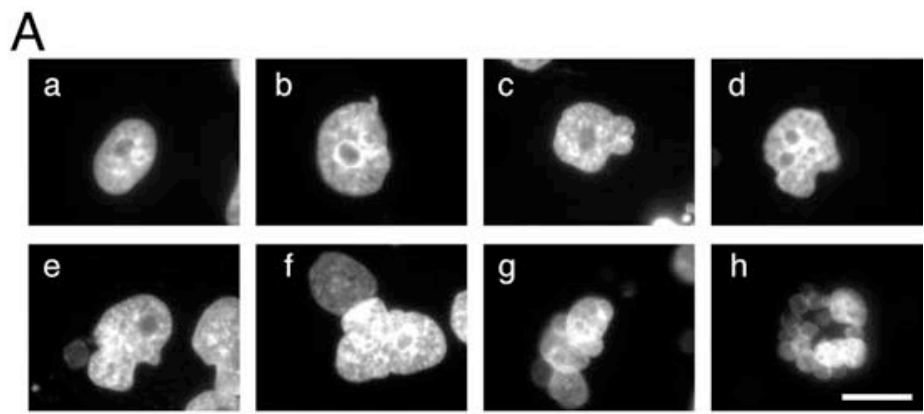

g
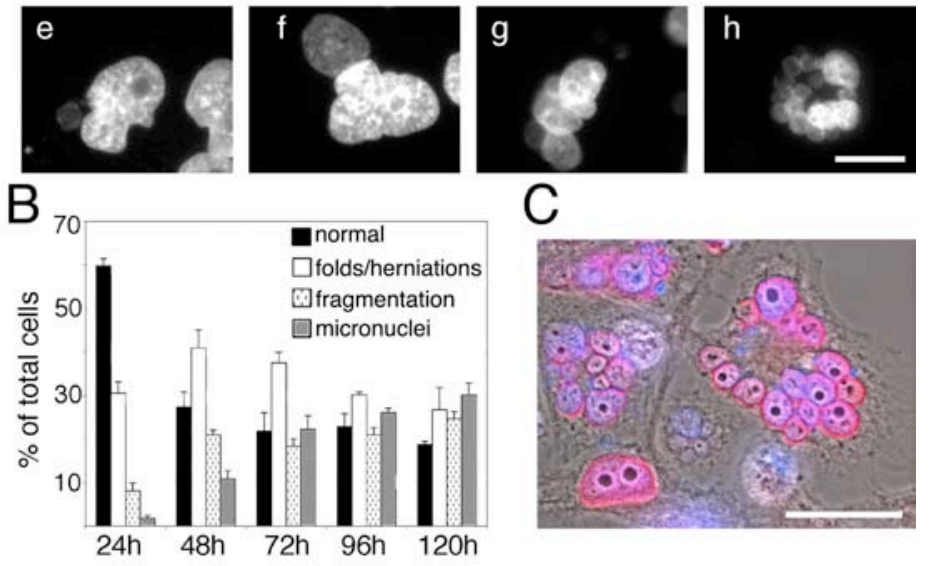

Figure 5: Silencing of FACE1 causes abnormalities in nuclear morphology. (A) Control HeLa cells (a) show a normal nuclear morphology while FACE1 silenced HeLa cells show a range of abnormal nuclear morphologies (b-h) when stained with Hoechst dye. The abnormalities included minor effects such as herniations in the nuclear periphery (b) and folds (c-f), or more severe effects such as lobules (f) and fragments $(\mathrm{g})$ as well as in some cells formation of micronuclei $(\mathrm{h})$. The number of cells with severe abnormalities increased significantly at later times after transfection. (B) Comparative statistics. Cells were divided into four classes. "Normal" nuclei have a morphology as in (a), "folds and herniations" as in (c-f), fragmentation as in $(\mathrm{g})$ and micronuclei as in (h). The percentage of "normal" nuclei decreased from $60 \%$ at 24 hours to $20 \%$ at 72 hours. Folds and herniations reached a maximum $(>40 \%) 48$ hours after transfection and then decreased slowly. The number of cells with micronuclei increased throughout the observation time and reached a maximum (>30\%) 120 hours post transfection. (C) Cells treated with FACE1 siRNAs in phase superimposed with prelamin A stain (red) and Hoechst DANN stain (blue) to show micronuclei. (Bar in A, C: $15 \mu \mathrm{m}$.)

\section{III.1.3 $\quad$ FACE1 knockdown caused aberrant mitosis and apoptosis}

A very striking change observed after knockdown of the FACE1 endoprotease was the accumulation of cells that are in aberrant mitosis (Fig. 6). Cells were arrested in early metaphase and displayed disturbed spindle architecture and abnormal chromosome congression (Fig. 6A). Analysis of FACE1 silenced cells by the TUNEL assay showed that following the aberrant mitotic arrest the cells in this 
population underwent apoptosis (Fig. 6B). Quantitation of cells in mitotic arrest and apoptosis are shown in Fig. 6C and D. After 24 hours $25 \%$ of the cells were arrested in mitosis and after 48 hours this value increased to a maximum of $35 \%$ (Fig. 6C). At later times the percentage of cells arrested in aberrant mitosis decreased. The percentage of cells that were apoptotic reached a maximum 72 hours post transfection with FACE1 siRNA (Fig 6D).

A
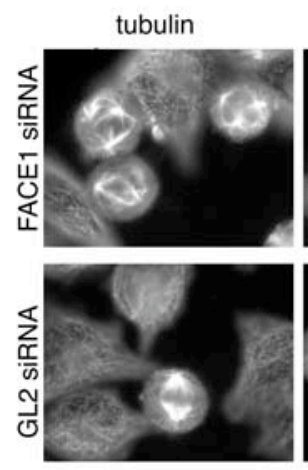

B

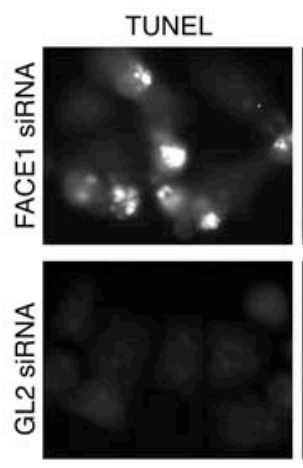

DNA
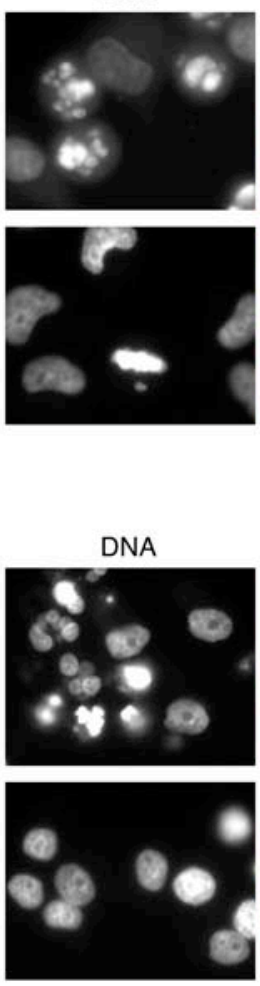

Merge
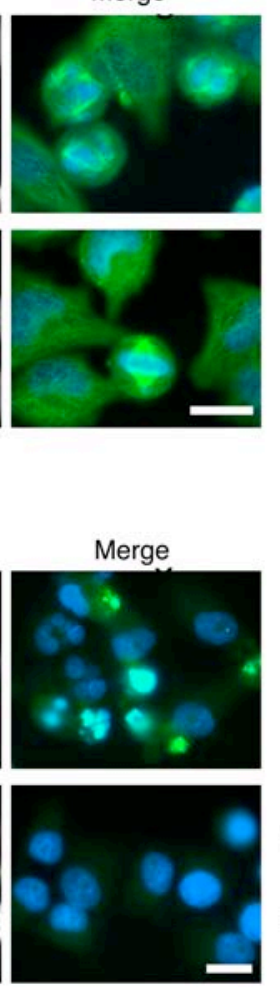

C
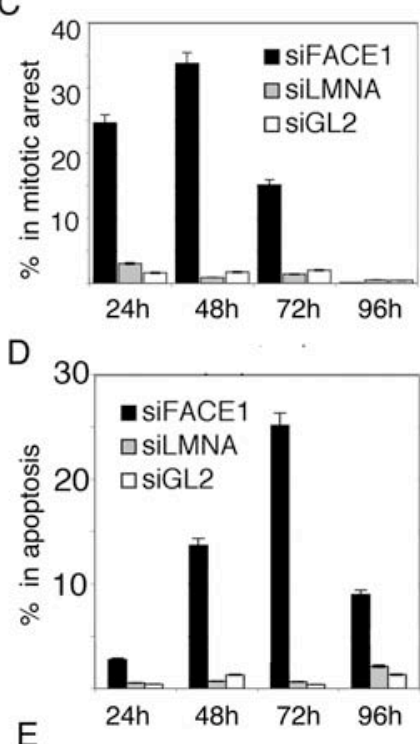

$\mathrm{E}$

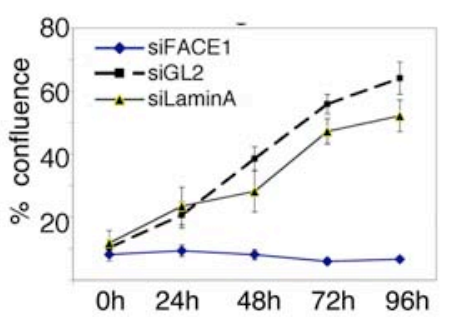

Figure 6: Aberrant mitosis and apoptosis are consequences of FACE1 knockdown.

(A) RNAi mediated knockdown of FACE1 led to mitotic arrest of HeLa SS6 cells already 24 hours after transfection. Arrested cells (top) displayed abnormal mitotic spindle architecture and disturbed chromosome congression. Control cells (bottom) transfected with GL2 siRNA showed normal mitosis. Cells were stained with $\square$ tubulin antibody and with Hoechst dye. (B) TUNEL assay on FACE1 silenced (top) and control cells (bottom) 72 hours after siRNA transfection shows that the mitotically arrested cells have entered apoptosis. (C) Quantitation of mitotic arrest after FACE1 silencing. The fraction of cells showing mitotic arrest increased with time after transfection, reaching a maximum of $35 \%$ of the total cell population at 48 hours. Cells transfected either with GL2 or with lamin A siRNA did not arrest in mitosis. (D) Quantitation of apoptosis after FACE1 silencing. The percentage of 
apoptotic cells reached a maximum of $25 \% 72$ hours after transfection and then decreased. Apoptosis was observed only in FACE1 silenced, but not in control (GL2 siRNA) or in lamin A siRNA transfected cells. (E) Cell growth measured with the CellScreen apparatus was reduced dramatically after silencing with FACE1 siRNA. (Bar: $15 \mu \mathrm{m}$.)

Measurements of cell growth were made with the Cell Screen System for the FACE1 siRNA transfected cells, as well as for GL2 and LMNA transfected cells (Fig. 6E). Cells transfected with FACE1 siRNA stopped growing abruptly. LMNA silenced cells showed a small reduction in growth rate (see also (Elbashir et al., 2002)) when compared to the GL2 control, but the change in growth rate was minor in comparison to that seen with the FACE1 silenced cells.

III.1.4 The phenotypic changes after FACE1 silencing are due to the increase in prelamin A

Double RNAi experiments were performed to see whether the phenotypic changes were due to the accumulation of prelamin A. Mitotic arrest and the changes in nuclear morphology were not observed, if lamin A was silenced 24 hours before the FACE1 knockdown (Fig. 7A, top). Thus when cells were stained with $\square$ tubulin antibodies, normal mitotic spindles were seen and mitotic cells with abnormal multipolar spindles were not found. In contrast, if FACE1 was silenced 24 hours before lamin A was silenced, results were similar to those reported above for FACE1 silencing alone in that accumulation of cells with aberrant mitotic arrest was observed 48 hours after the first transfection (Fig. 7A, bottom). This data strongly suggests that the accumulation of prelamin A is the cause for effects seen in HeLa cells.

The bDNA assay was used to confirm that both FACE1 and lamin A were knocked down when a double RNAi experiment was performed. Examination of the 
results in Fig. 7B show that in the double knockdown experiment leading to normal mitosis, i.e. knockdown of lamin A followed 24 hours later by knockdown of FACE1, mRNA levels are reduced to $20 \%$ when assayed 24 hours after the second transfection (*, Fig. 7B). If FACE1 is knocked down 24 hours prior to LMNA, again a reduction in mRNA levels for both proteins is seen, although in this case the FACE1 mRNA is more strongly reduced than is the LMNA mRNA.

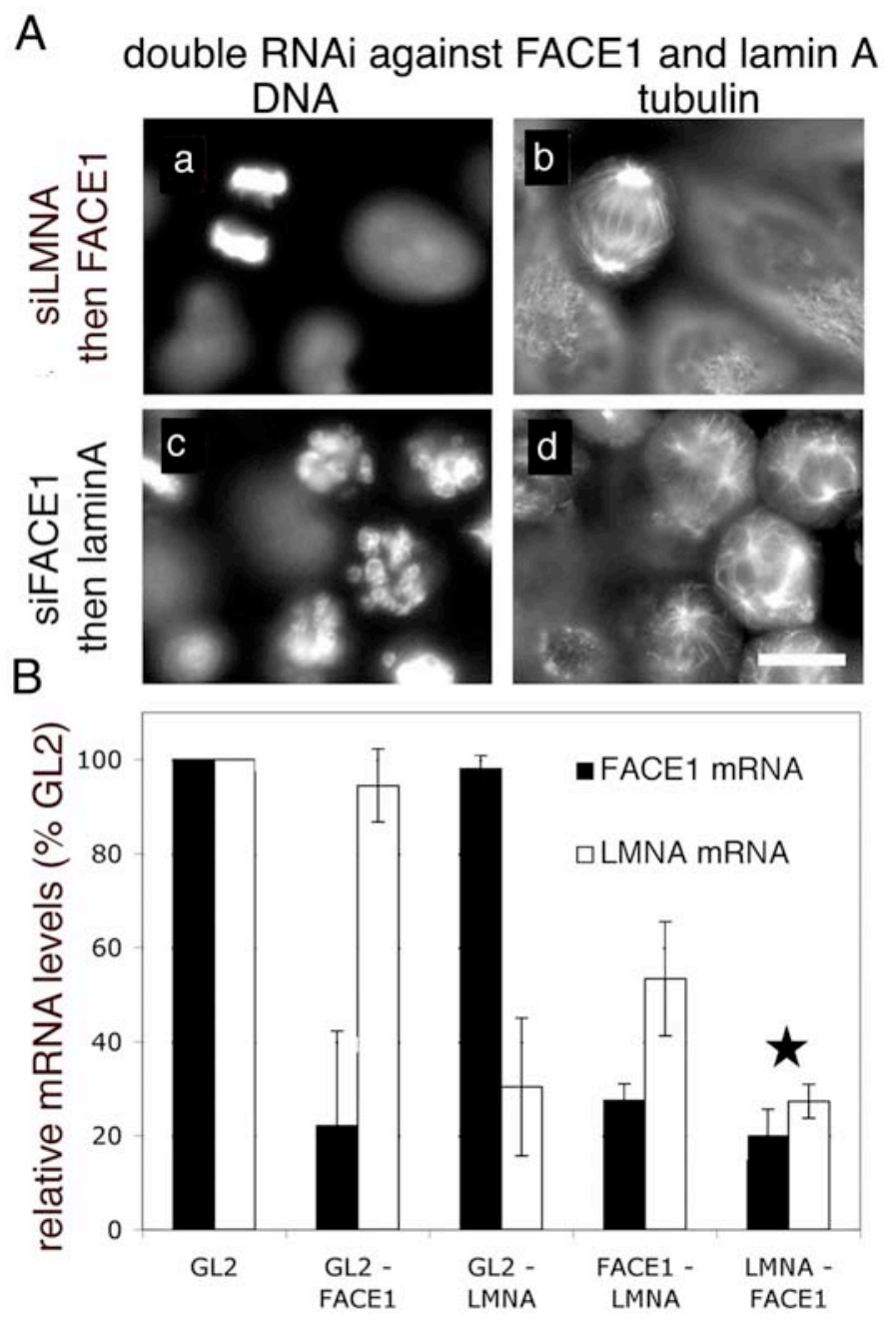

Figure 7: Double RNAi suggests prelamin A is responsible for phenotypic changes after FACE1 silencing. (A) Silencing of lamin A prior to FACE1 prevented phenotypic changes. Human HeLa SS6 cells were transfected with the siRNA specific for the first target and after 24 hours with the second siRNA. Cells were stained for $\square$ tubulin and for DNA 24 hours after the second transfection. (a, b) Knockdown of lamin A before FACE1 did not cause abnormal mitosis, apoptosis or changes in the nuclear morphology. (c, d) Silencing of FACE1 prior to silencing of lamin A led to aberrant mitotic arrest. (B) Successful transfection in the double knockdown experiments was confirmed by the branched DNA assay. Data represent 
the relative mRNA levels 24 hours after the second transfection for the LMNAFACE1 combination and for the FACE1-LMNA combination. Double siGL2 transfection was used for normalisation (GL2). That the second transfection was effective was further confirmed by transfecting either FACE1 or lamin A siRNA 24 hours after siGL2 delivery (GL2 - FACE1 and GL2 - LMNA). (Bar in A: $10 \mu \mathrm{m}$.)

Additional confirmation that the phenotypic changes after FACE1 silencing are due to the increase in prelamin A, comes from the HL60 cell line. This human promyelocytic cell line does not express the lamin A gene. When HL60 cells were transfected by electroporation with FACE1 siRNA, no growth retardation, mitotic arrest or effects on nuclear architecture were observed (Fig. 8).

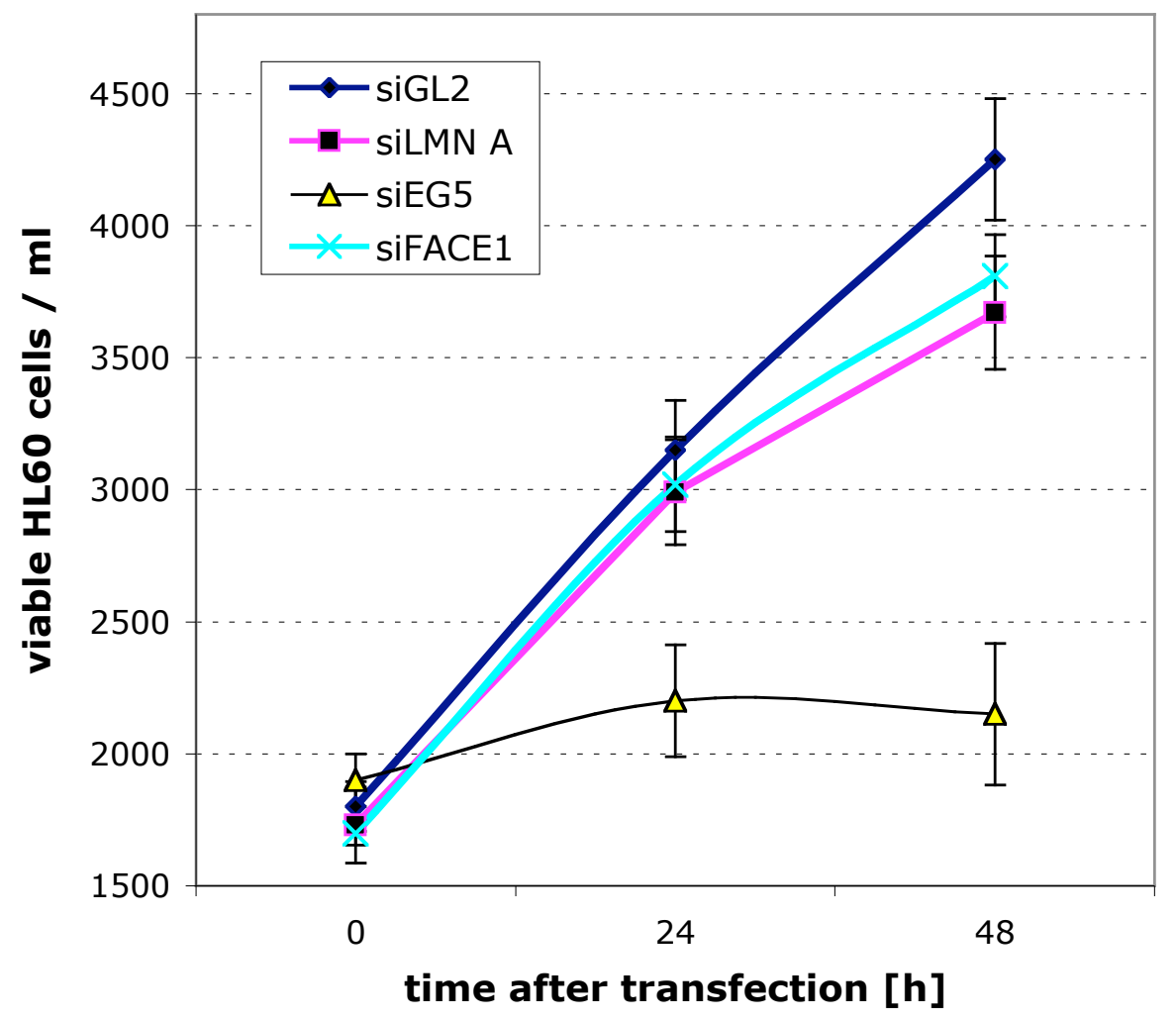

Figure 8: Growth rate determination of HL60 cells after transfection of different siRNAs. Silencing of neither FACE1 nor lamin A/C siRNAs resulted in significantly reduced growth rates when compared to GL2 siRNA negative control. 
In contrast transfection of siRNA against $\mathrm{Eg} 5$ induced mitotic arrest and dramatically decreased cell growth.

The effectiviness of the FACE1 siRNA, which was used 24 hours after the lamin A siRNA, was confirmed by the branched DNA assay. In other double RNAi experiments (data not shown) we have used also siRNA for the mitotic motor protein Eg5 or for $\square$-actin and observed the phenotypes earlier described for single RNAi experiments (Harborth et al., 2001). Thus the consecutive use of two siRNAs affecting different mRNAs is a very useful tool in RNAi technology.

\section{III.1.5 Location of FACE1 in HeLa cells}

The immunofluorescence results in Fig. 9 show that in HeLa cells FACE1 is located in spots at the nuclear membrane and in the endoplasmic reticulum. Staining of the endoplasmic reticulum was confirmed by first staining live cells with 1.5

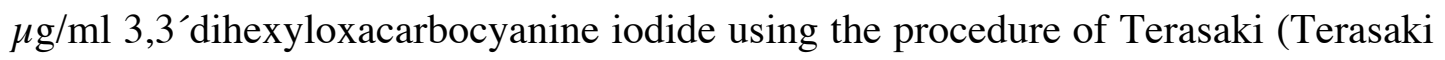
et al., 1984) and then with FACE1 antibodies. In cells transfected with FACE1, siRNA staining at the nuclear membrane and in the endoplasmic reticulum is very strongly reduced 48 hours after transfection (Fig.9B). 
A
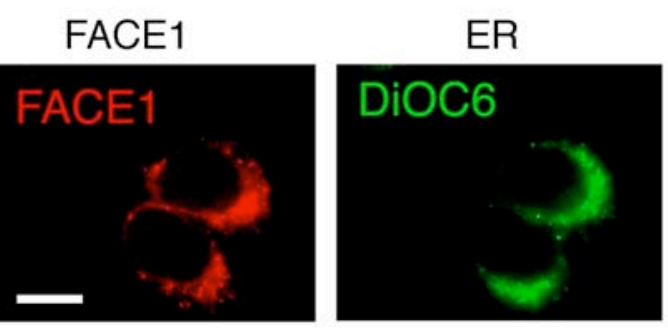

B

siGL2

SiFACE1
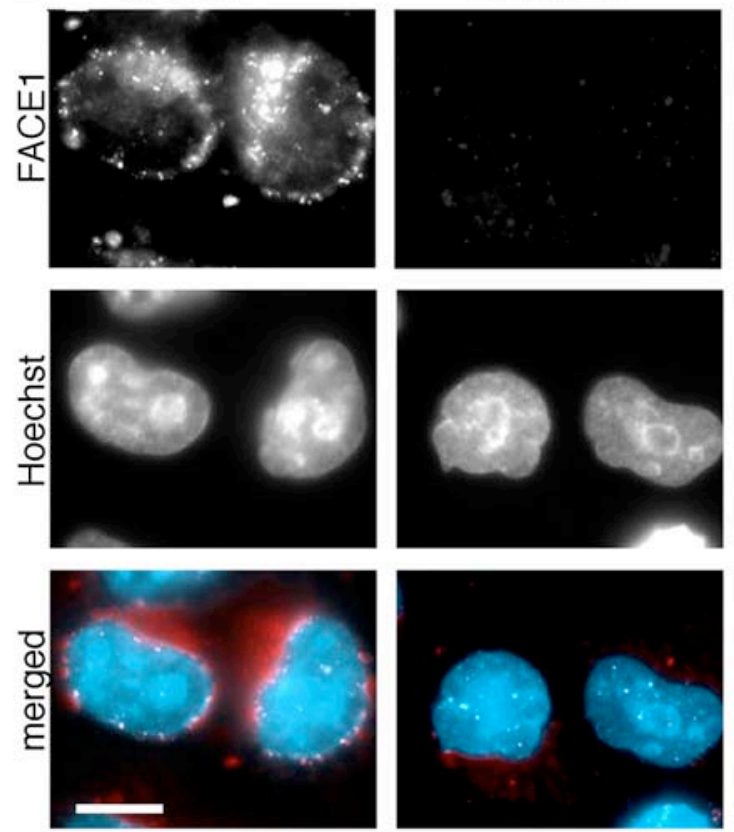

Figure 9: Immunofluorescence detection of FACE1 in the endoplasmic reticulum and the nuclear periphery (A) HeLa cells were stained with dihexyloxacarbocyanine iodide to label the endoplasmic reticulum and then with the rabbit FACE1 antibody against residues 296-313. FACE1 is seen both in the endoplasmic reticulum identified by $\mathrm{DiOC}_{6}$ staining and is also seen in spots at the nuclear periphery. (B) Immunofluorescence with the FACE1 specific antibody 48 hours after transfection of FACE1 or GL2 siRNA demonstrates silencing of FACE1 in cells transfected with FACE1 siRNA, but not in cells transfected with GL2 siRNA. (Bar: $10 \mu \mathrm{m}$.)

\section{III.1.6 Overview of results from RNAi against FACE1}

Successful knockdown of FACE1 was demonstrated on protein level by

immunofluorescence and western blot, on RNA level by the branched DNA assay.

Knockdown of the endoprotease led to accumulation of unprocessed prelamin

A and its translocation from subnuclear foci to the nuclear envelope. 
Two phenotypes have been observed; in the first cells diplayed aberrant mitosis early after transfection of FACE1 siRNA and subsequently underwent apoptosis, in the second cells displayed increasingly severe abnormalities in nuclear architecture.

FACE1 knockdown in cells negative for lamin A/C (i.e. HeLa SS6 cells after lamin A/C silencing and HL60 cells) indicated that the accumulation of prelamin $A$ is responsible for the phenotypic changes.

FACE1 was shown to locate to the nuclear periphery and the endoplasmic reticulum in control cells. 


\section{III.2 Human targets 2; the mitotic spindle associated protein astrin and mitosis related proteins}

\section{III.2.1.1 Astrin is an essential protein of the spindle}

The major aim of this part of the thesis was to investigate potential functions of the novel protein astrin using the recently introduced technique of siRNA mediated gene silencing. Using this reverse genetics approach it could be successfully monitored that astrin is knocked down in cells that were transfected with siRNAs targeting astrin mRNA. Immunofluorescence and apoptosis detection assays demonstrated that astrin is an essential protein and cells lacking it are mitotic arrested and subsequently undergo apoptosis. The knockdown cells showed a rounded morphology in phase contrast microscopy and an aberrant mitotic arrest in immunofluorescence with astrin specific antibodies. Immunofluorescence showed that at least one siRNA duplexes targeting astrin mRNA led to highly efficient knockdown of the gene leading to a phenotype with highly disordered $\square$ tubulin distribution instead of properly formed spindle apparatus and a dispersed chromatin distribution where the metaphase plate normally is established.

The siRNA sequence for targeting human astrin was from position 2639 to 2661 relative to the first nucleotide of the start codon. Control experiments used a siRNA sequence for firefly luciferase (pGL2). Phase microscopy showed that with time the astrin siRNA treated cells became growth arrested and that an increasing number of cells rounded up. 


\section{III.2.1.2. $\quad$ Cells displayed disturbed spindle alignment after astrin silencing}

At 44 hours after transfection more than $50 \%$ of the cells had rounded up. Indirect immunofluorescence microscopy was performed at this time using double staining with affinity purified rabbit antibodies to astrin and a murine monoclonal $\square-$ tubulin antibody (Fig. 10A). Astrin silencing resulted in a strong decrease in astrin staining. Microtubule distribution and morphology of astrin silenced interphase cells were not affected and did not show any differences to control siRNA treated cells. However, mitotic cells showed a remarkable phenotype with aberrant mitotic arrest and multipolar and highly disordered spindles indicating that astrin function is essential for progression through the cell cycle. Chromosomes did not congress to the spindle equator and remained dispersed (Fig. 10A,B). Staining of astrin silenced cells with -tubulin antibody to visualize centrosomes clearly indicated fragmentation of centrosomes. - - tubulin was present in all poles of the aberrant multipolar spindles after astrin depletion (Fig. 10B). Astrin silencing was also confirmed by immunoblotting experiments. While cells transfected with the luciferase siRNA revealed a strong astrin band at $140 \mathrm{kDa}$ the silenced cells lacked a recognizable signal (Fig. 10C). 


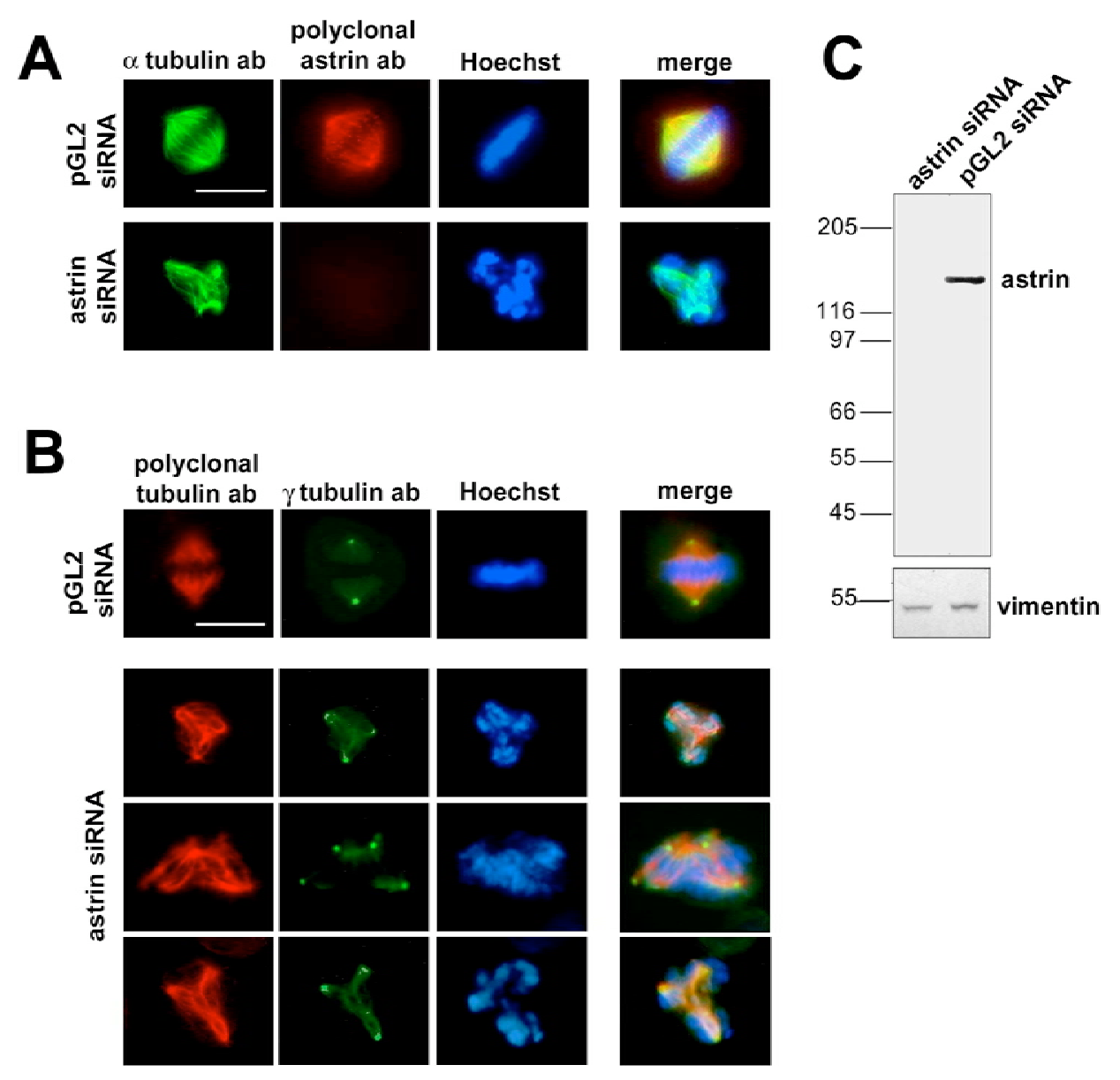

Figure 10: siRNA induced knockdown of astrin in HeLa cells. Cells were transfected with the luciferase control siRNA (pGL2 siRNA) or with the astrin specific siRNA (A). After 44 hours cells were stained for $\square$-tubulin, astrin and DNA (Hoechst). Note the loss of astrin from the abnormal mitotic figures induced after astrin depletion. (B) After 44 hours cells were stained for $\square$ tubulin, $\square$-tubulin and DNA (Hoechst). Astrin depletion affects chromosome congression and proper spindle formation. No normal metaphase cells were found. Bars in (A) and (B): 15 $\mu \mathrm{m}$. (C) Silencing of astrin was confirmed by the immunoblots. The lower parts show comparable reaction of the blots of control and astrin siRNA treated cells with vimentin antibody. 

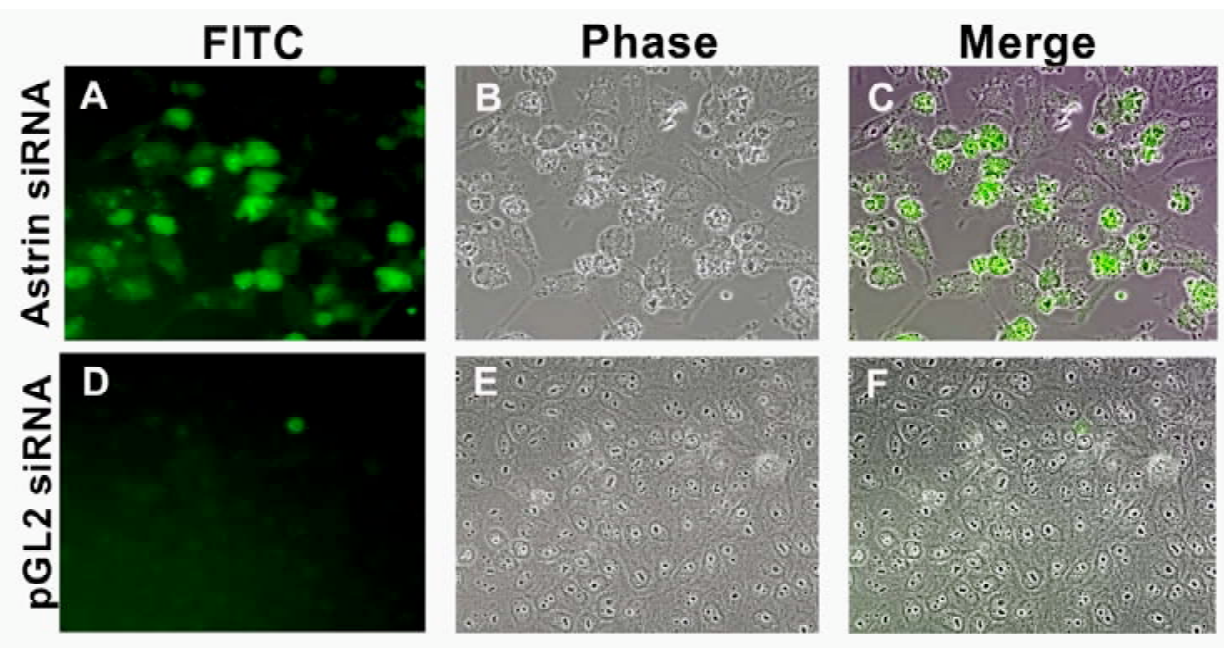

Fig. 11: Astrin silencing induces apoptosis. The TUNEL assay shows that many of the cells transfected with the astrin siRNA undergo apoptosis (green colour) at 60 hours post-transfection (A-C). Cells transfected with the luciferase siRNA (pGL2) continue to grow (D-F). A single apoptotic cell was detected in this field. Phase image and fluorescent image arising from the incorporation of FITC labeled deoxynucleotides are superimposed (for details see Methods).

\section{III.2.1.3 $\quad$ Two additional siRNAs against astrin did not produce an efficient}

\section{knockdown}

Three different siRNAs against astrin have been applied, but only one was efficient at inducing RNAi. Results described above were obtained with the functional siRNA astrin2 (see Table1). The other two siRNAs did not lead to astrin knockdown and the expecetd phenotype. Results concerning knockdown efficiencies and apoptosis induction of the three siRNAs against astrin are summarized in Figure 12. The relevance of the siRNA structure will be discussed in section V.3. 

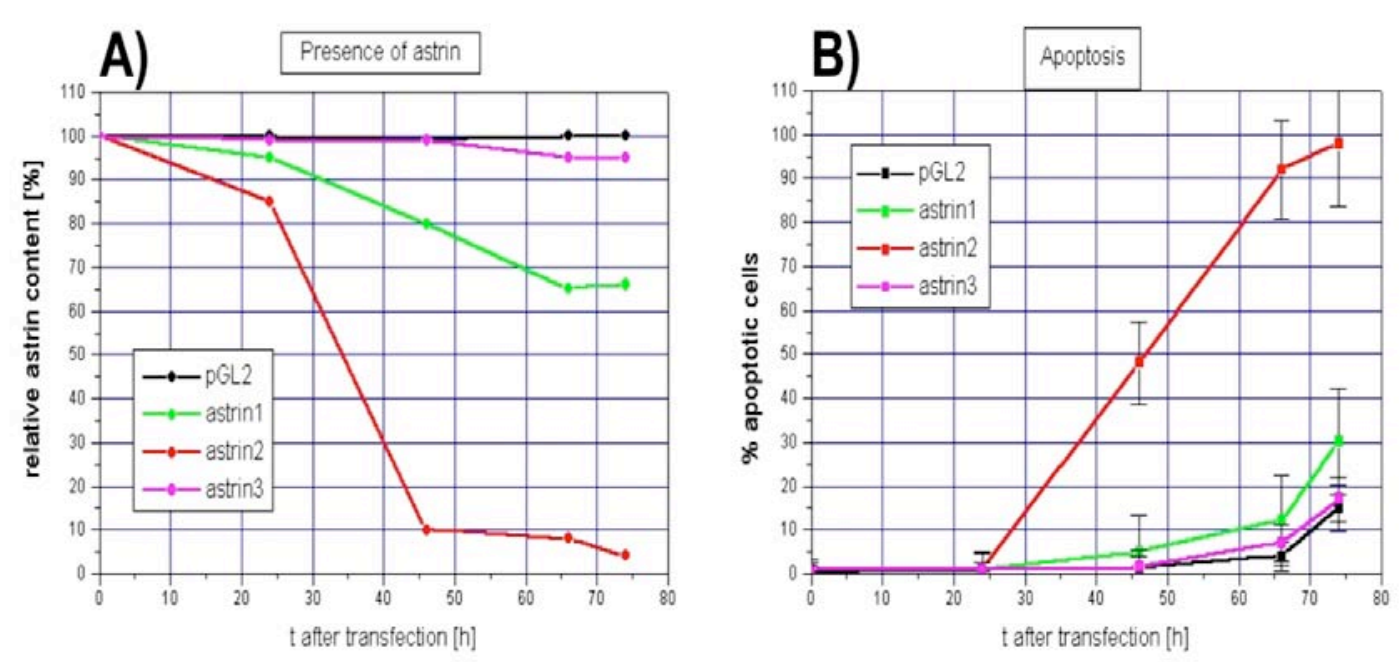

Figure 12: Graphs visualising data from immunofluorescence and apoptosis detection experiments with different astrin siRNA duplexes. The decreasing reactivity of astrin antibodies in immunofluorescence with astrin or pGL2 siRNA transfected HeLa SS6 cells is presented in (A). The astrin2 duplex (red) was the most efficient in target silencing leading to $90 \%$ decrease of astrin positive cells after 46 hours. astrin1 (green) was less efficient and showed the maximal silencing ( $35 \%)$ after 66 hours while astrin3 (violet) did not show significant differences from control siRNA transfected cells (black). Cells in (B) were subjected to the TUNEL test and observed 24, 46, 66 and 74 hours after transfection using fluorescence microscopy. Nearly $50 \%$ of the astrin 2 treated cells were apoptotic after 46 hours and after 74 hours virtually all cells were dead or dying. Cells transfected with astrin 1 siRNA duplexes also underwent apoptosis, but at a much slower rate. pGL2 and astrin3 transfected cells started to enter apoptosis after 66 hours.

III.2.2 Silencing of mitosis related proteins

Since mitosis is one of the most important processes in the cell various targets from the large group of mitosis related proteins were selected as targets for gene silencing to learn more about this machinery. The first group of mitosis related target genes covers different motor proteins. These are involved in microtubule sliding during nuclear fusion or division, while others mediate chromosome disjunction in early mitosis or play key roles in the establishment of the mitotic spindle apparatus. All these functions are related to movements of organelles or chromosomes along microtubules. Molecular motors move directionally to either the plus or the minus 
ends of microtubules. The first motor protein to be silenced was Eg5. Silencing of Eg5 showed a strong effect after the knockdown, leading to monopolar mitotic spindles and subsequent apoptosis. Currently I am working on the motor proteins HSET, MCAK, KIAA0622, which are kinesin-like proteins. Kinesin-like proteins generally have a mechanochemical domain in their amino-terminal region. Silencing of the other kinesin-like motor proteins, i.e. HSET (with a mechanochemical domain in its C-terminal portion), MCAK, KIAA0622 leads to mitotic arrest followed by apoptosis.

Additional structural and linker proteins were silenced. Knockdown of the checkpoint proteins securin and separin led to metaphase arrest and apoptosis. KIF4 showed an apoptotic phenotype, astrin was already described (see III.2.1).

The microtubule destabilizing proteinsstathmin and katanin were also silenced. Katanin is a microtubule severing protein, which consists of the catalytic subunit p60 and the subunit p80. Knockdown of either of the subunits led to aberrant microtubules and in some cases to apoptosis. Stathmin destabilizes microtubules by binding the $\square$ tubulin subunits of free $\square / \square$ dimers. Loss of stathmin led to aberrant microtubules and diverse types of aberrant mitotic spindles. The last target gene is the antigen recognized by the monoclonal antibody KI 67 . The gene is known to be expressed at high levels in proliferating cells and is therefore used as a tumor marker. Silencing of Ki67 did not lead to a sharp phenotype, but a slightly decreased growth rate of Ki67 siRNA transfected cells could indicate a $\mathrm{G}_{0}$ arrest.

A summary of results of these silencing experiments is shown in Table 3. 
Table 3: Selected targets for RNA interference and a survey of the effects after silencing

\begin{tabular}{|c|c|c|c|c|}
\hline Target gene & $\begin{array}{l}\text { silencing } \\
\text { (IF, WB) }\end{array}$ & effect & Essential & Phenotypic change \\
\hline HSET & & ++ & + & Arrest \\
\hline Eg5 & & +++ & + & Arrest, monopolar spindles \\
\hline KIF4 & & + & + & Aberrant mitosis \\
\hline CENP-E & & ++ & + & mitotic arrest \\
\hline Astrin & + & +++ & + & Aberrant mitosis \\
\hline KIAA0622 & & + & + & Aberrant mitosis \\
\hline Securin & & ++ & + & Arrest \\
\hline Separin & & + & $(+)$ & Arrest \\
\hline Sccl & & +++ & + & Arrest \\
\hline Smc1 & & +++ & + & Arrest \\
\hline Smc3 & & +++ & + & Arrest \\
\hline MCAK & & + & + & Arrest, aberrant mitosis \\
\hline Katanin p60 & + & ++ & + & Aberrant telophase \\
\hline Katanin p80 & & $(+)$ & $?$ & \\
\hline stathmin & + & ++ & + & Aberrant spindle morphology \\
\hline $\mathrm{Ki}-67$ & + & +++ & - & $\begin{array}{l}\text { growth retardation, reduced } \\
\text { mitosis }\end{array}$ \\
\hline
\end{tabular}

(Note: + in the silencing column means that silencing was confirmed with specific antibodies against the target protein. The number of + in the column effect refers to the relative number of phenotypic cells; $+=20 \%$ or less, $++=20-50 \%,+++=>50 \%$.)

III.2.2.1 Silencing of kinetochore proteins and the cohesion complex

The cohesion complex is largely represented by the proteins smc1, smc3 and scc1, forming a tight ring around the centromeres mitotis sister chromatids. Silencing of each of these molecules led to severe effects on the ability of proper mitosis and viability of the cells. Silencing of both smc1 and smc3 caused mitotic arrest in metaphase. Cells were transfected with siRNAs against smc1 or smc3 and displayed disordered spindle microtubules in a minor portion of cells (Fig. 14) and disturbed chromosome congression. The same abnormalities were observed after transfection of siRNA targeting scc1, wich serves as a linker between smc1 and smc3, thereby closing these securing ring around centromeres. Silencing of the adhesion complex proteins caused phenotypic changes early ( $<24$ hours) after transfection, indicating the cell cycle dependent expression. The severe effects silencing of scc1, smc1 and 
smc3 on cell cycle progression is reflected in growth rates of the cells (Fig. 13) Cells silenced for smc1 and smc3 showed a strong decrease in growth already 24 hours after siRNA transfection. Scc1 knockdown cells proliferated normally until 48 hours after transfection before these also decreased growth and cell density.

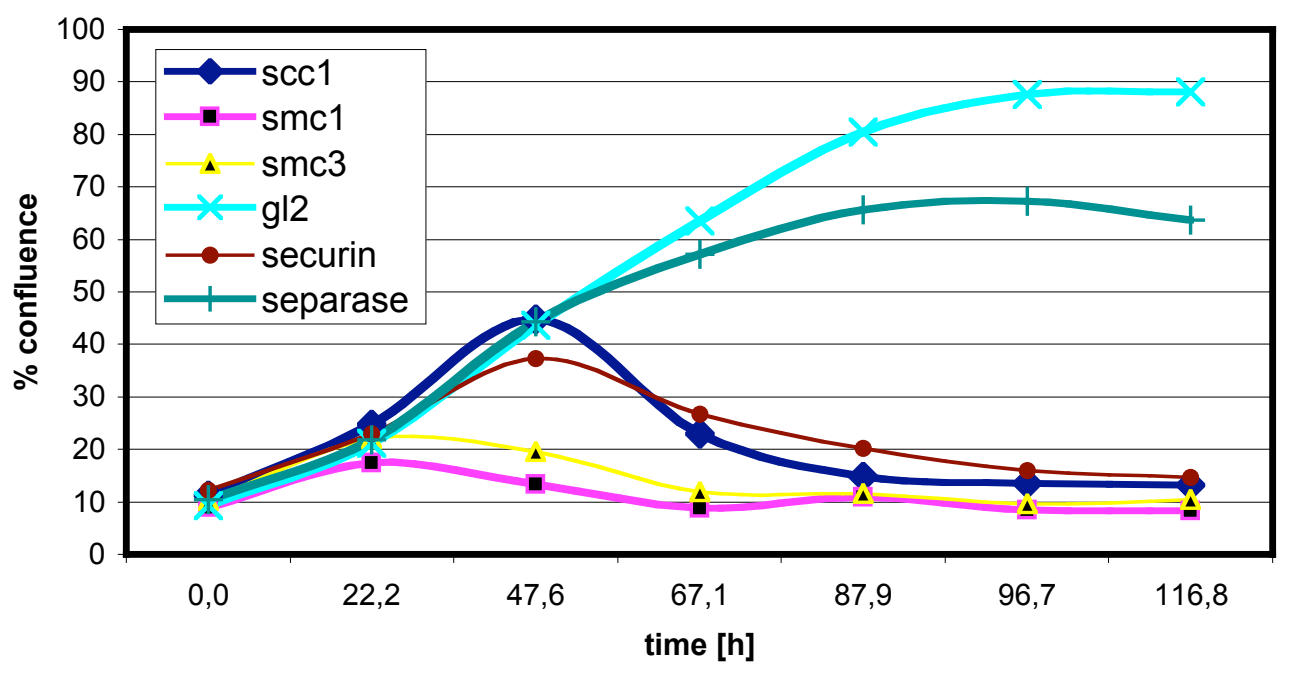

Figure 13 Growth rates of cells after transfection of siRNAs against kinetochore related proteins. HeLa SS6 cells grown on coverslips were examined by the CellScreen analysis system. Silencing of smc1 (pink) and smc3 (yellow) leads to a decrease in cell density already after 22 hours. Silenced cells cannot complete the first round of mitosis after transfection. Scc1 (dark blue) and securin (brown) knockdown affected cell growth after 48 hours.

The proteins scc1, smc1 and smc3 were also shown to be essential for mitosis and cell survival. These three proteins can be expected to be involved in kinetochore attachment.

The enyzme separase is expected to be responsible for cleaving the centromeric ring when proper kinetochore alignment and orientation is established. In this case securin interacts directly with scc1 and allows recognition by separase. Securin was also knocked down. 

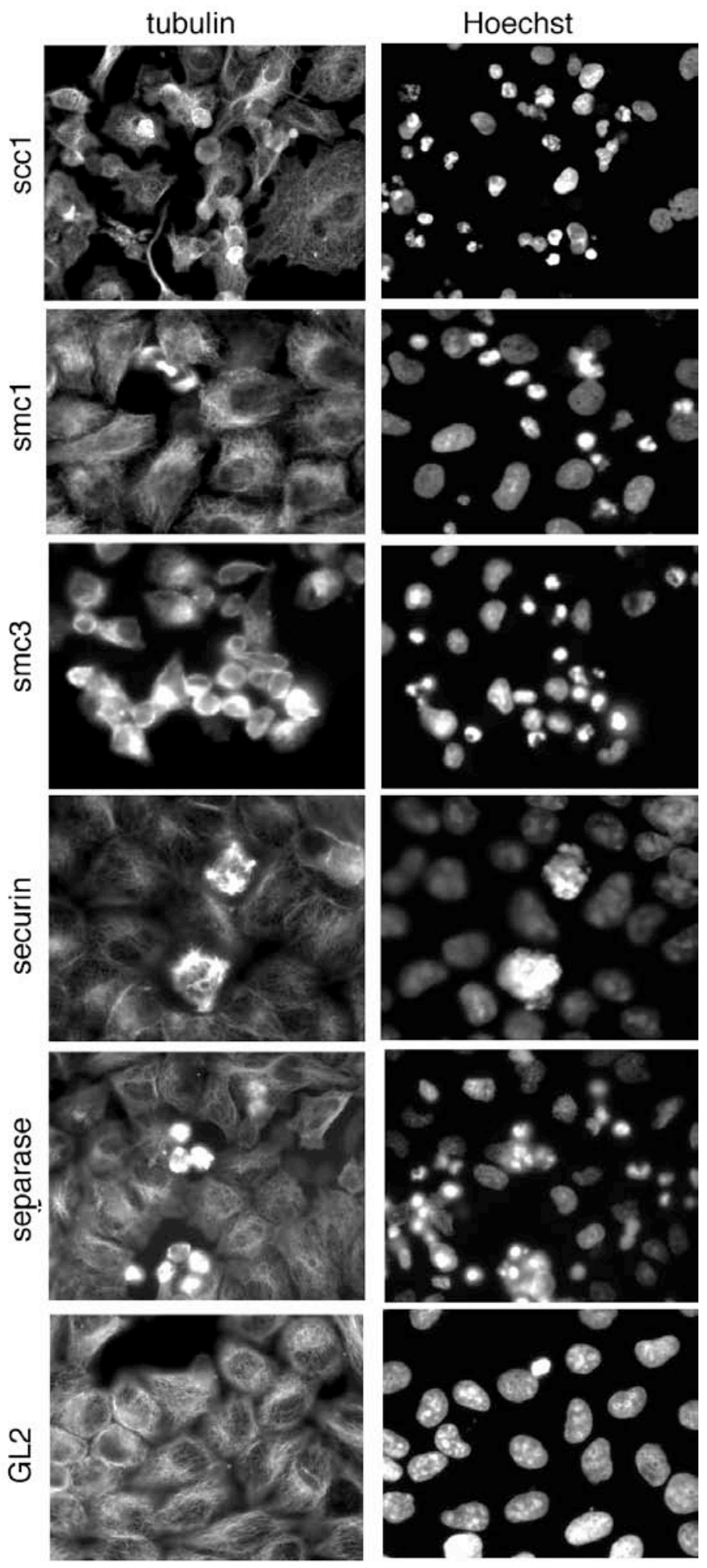

Figure 14: Immunofluorescence micrographs afetr RNAi against kinetochore proteins. SiRNAs against scc1, smc1, smc3, securin and separase were transfected into HeLa SS6 cells. SiGL2 served as a negative control. Cells were stained with an $\square$ tubulin antibody 44 hours after transfection. DNA was visualized with Hoechst 33342 dye. Chromosome congression was disturbed after knockdown of each of the kinetochore related proteins. Note the aberrant microtubules distribution after silencing of scc1, smc3 and securin. (Magnification x 400) 


\section{III.2.2.2 $\quad$ Silencing of kinesin related proteins.}

SiRNAs against the kinesin related motor proteins HSET, KIF4, TPX2, MCAK and Eg5 were transfected into HeLa SS6 cells. In addition, the cyclin dependent kinase 1 (cdk1) was silenced by RNAi. Cells transfected with the GL2 siRNA served as negative control. Silencing of all proteins mentioned above led to growth inhibition early after transfection. Only cells that were silenced for KIAA0622 grew weakly before the cell density decreased again after 67 hours (Figure 15).

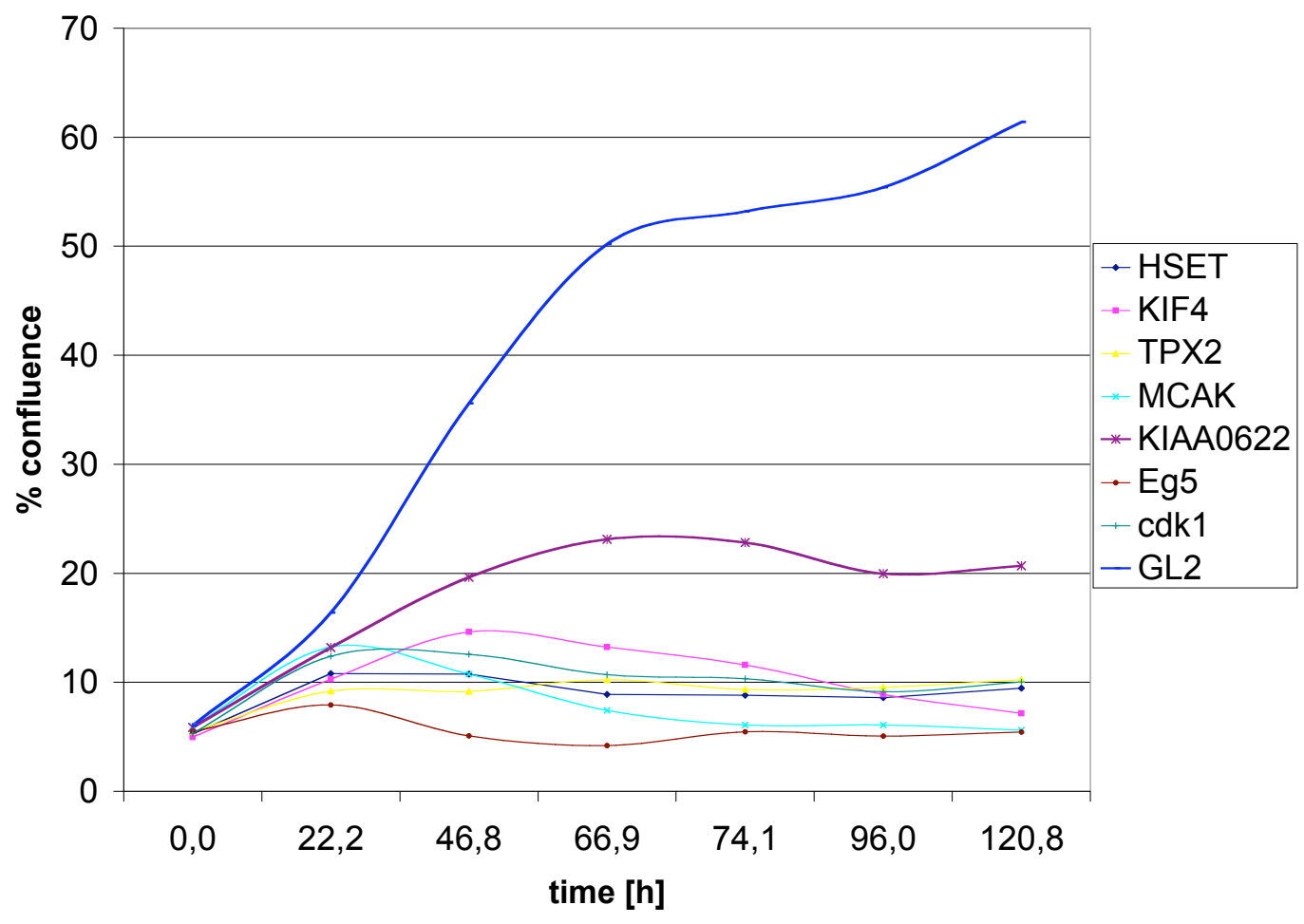

Figure 15: Cell growth after transfection of siRNAs against mitotis related proteins. HeLa cells were transfected with siRNAs and subjected to growth rate measurements by the CellScreen apparatus. An inhibition of cell growth was observed for all silenced proteins.

Indirect immunofluorescence microscopy with an antibody against $\square$ tubulin revealed aberrant mitosis after silencing of the kinesin related mtor proteins (Fig. 
16). Cells displayed highly disordered mitotic spindle microtubules and the chromosome congression was disturbed after silencing of MCAK, HSET and KIF4.
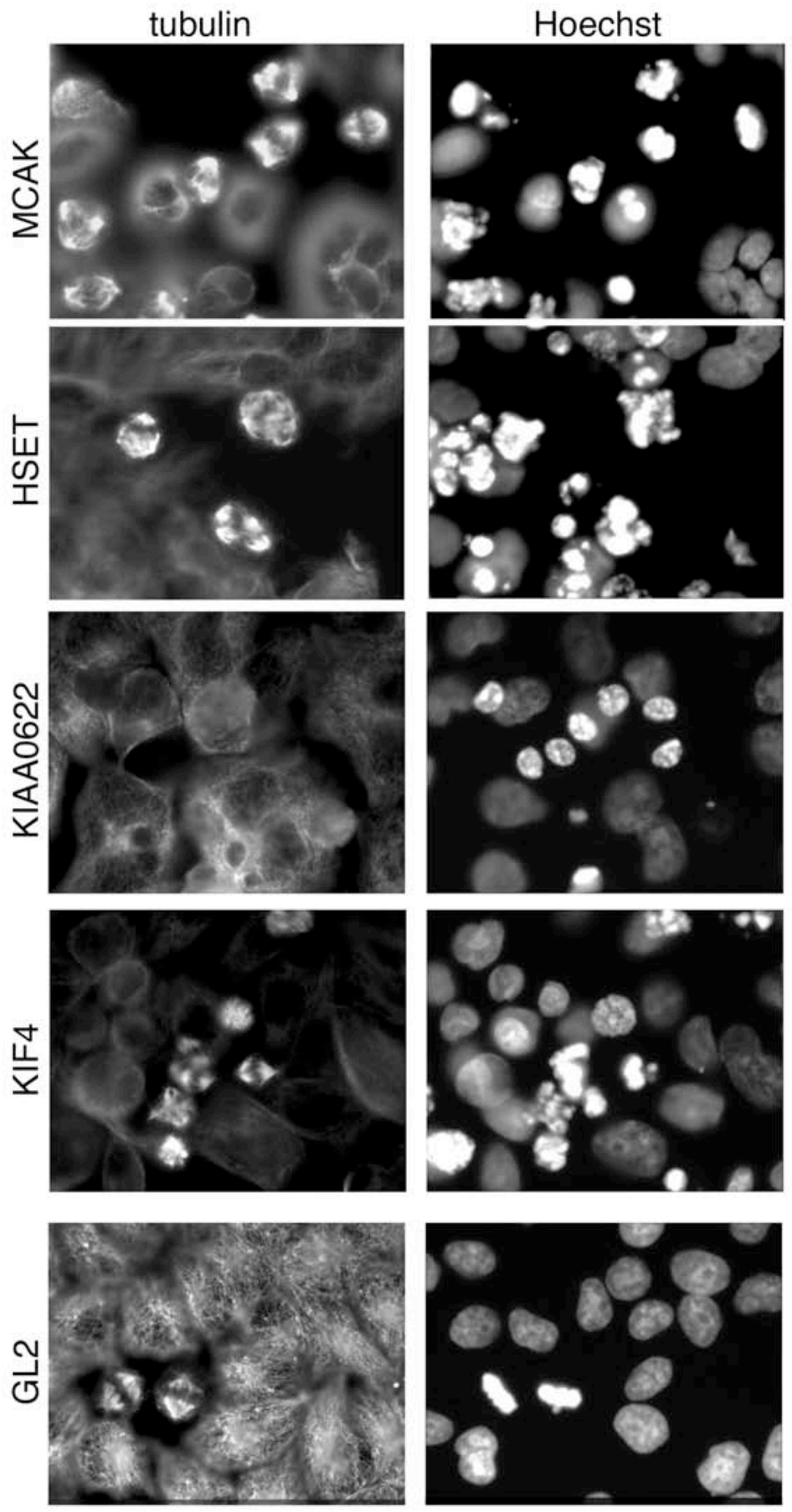

Figure 16: Cells displayed impaired growth after silencing of kinesin related motor proteins. HeLa cells were seeded on coverslips and transfected with siRNAs. 44 hours after transfection cell were stained with $\square$ tubulin antibody (DM1A) and the DNA was visualized with Hoechst 33342 dye. Note the aberrant mitotic cells after silencing of KIF4, HSET and MCAK. These cells display dioredered spindles and 
disturbed chromosome congression. Cells silenced for KIAA06 22 displayed a different microtubule and chromosome distribution with multinucleated cells.

\section{III.2.2.3 Silencing of stathmin and katanin}

The last set of siRNAs in this section of the thesis included the microtubule destabilizing proteins stathmin and katanin. Stathmin destabilizes microtubules by bindin free dimers of $\square / \square$ tubulin while katanin is the only protein known to actively cleave microtubules in an ATP dependent process. Katanin consists of two subunits, katanin p80 with unknown function, which is connected to a katanin p60 trimer that is catalytically active. Silencing of both stathmin and katanin p60 led to growth inhibition of transfected HeLa cells. Katanin p80 knockdown did not cause clear effects on cell growth (Fig. 17).

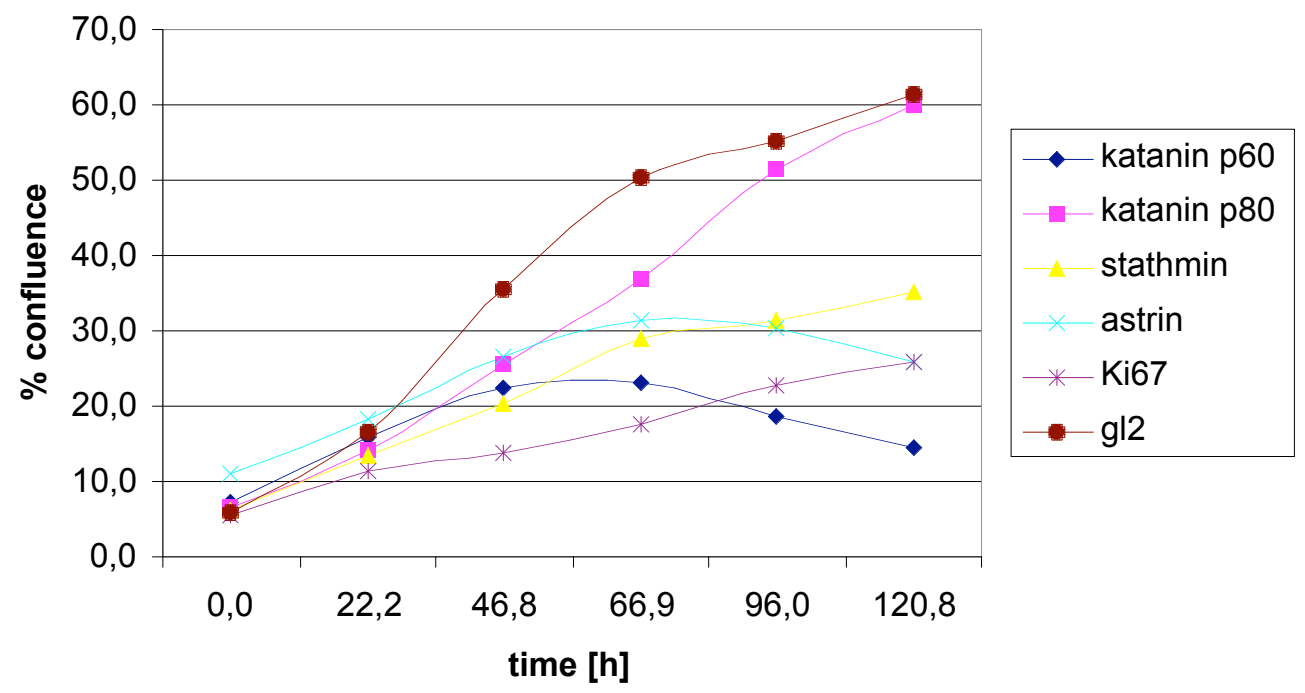

Figure 17: RNAi against the microtubule destabilizing proteins stathmin and katanin. Growth curves for silencing of astrin and Ki67 are also shown in this figure. GL2 is used as control. Cell Growth after siRNA transfection was as described before. 
III.2.3 Overview of results from RNAi against astrin and mitosis related proteins

The human coiled coil protein astrin was successfully silenced, resulting in a phenotype with cell displaying mutipolar mitotic spindles and disturbed chromosome congression.

Astrin silencing led to apoptotic cell death.

$>$ Silencing of the kinetochore proteins scc1, smc1, smc3, securin and separase resulted in impaired cell growth and growth retardation.

Knockdown of kinesin related motor proteins (i.e. HSET, MCAK, KIAA0622, and KIF4) showed a phenotype with highly disturbed mitotic microtubules.

Silencing of the kinesin motor proteins and the mitosis related proteins cdk1, TPX2 led to growth retardation in affected HeLa cells.

RNAi against the microtubule destabilizing proteins katanin and stathmin induced decreased growth rates, which is also true for RNAi against Ki67 


\section{III.3 Anatomy of siRNAs}

In a study silencing with only 5'-phosphorylated antisense (as) RNAs the most efficient as RNA turned out to be self complementary (Martinez et al., 2002). This finding was the motivation to further investigate gene silencing with antisense RNAs and palindromic RNA homoduplexes. The aim of this part of the thesis was to determine and compare possibilities of silencing endgenous genes with homoduplexes of asRNAs, asRNAs alone, heteroduplexes of two asRNAs targeting different regions on one gene and finally dual targeting with heteroduplexes targeting two different genes i.e. lamin $\mathrm{A} / \mathrm{C}$ and emerin. The lamin $\mathrm{A} / \mathrm{C}$ cDNA sequence was screened for regions interesting for either approach. Four palindromic regions, two pairs of complementary target regions and four complementary regions with one target region on lamin $\mathrm{A} / \mathrm{C}$ and the second on emerin were used in this study.

The possibility of using homodimeric ssRNA in gene silencing was investigated. Based on the knowledge of the near palindromic features of the siRNA targeting position 608 in the lamin $\mathrm{A} / \mathrm{C}$ mRNA, as described in the introduction, further self complementary regions of $20 \mathrm{nt}$ in length were identified along the lamin A/C cDNA sequence. Corresponding siRNAs were synthesized and contained 5'phosphate groups.

Due to the limited number of available palindromes an alternative strategy was developed. The aim of this approach was to find siRNA target regions on the lamin $\mathrm{A} / \mathrm{C}$ mRNA, that are complimentary to each other. This two target site strategy was created to learn more about the rational design of functional siRNAs. The idea behind it was that the main criterion that determines if a siRNA is an efficient 
silencer or not, is basically the incorporation of the right strand of the siRNA into RISC. If the sense strand is incorporated, then silencing will not be observed, but if the antisense strand goes into RISC, the siRNA should act as an efficient silencer. If both strands have a very specific target region on the target mRNA, both can act as the antisense strand for RISC formation and silencing. The strategy is depicted in figure 18.

The two target site approach was expanded to a second gene. The complementary antisense RNAs were designed to target two different genes. Thus asRNA-X targets mRNA-X while asRNA-Y targets mRNA-Y. This would allow the use of a single dsRNA to silence two genes at the same time.

A) palindromic siRNA

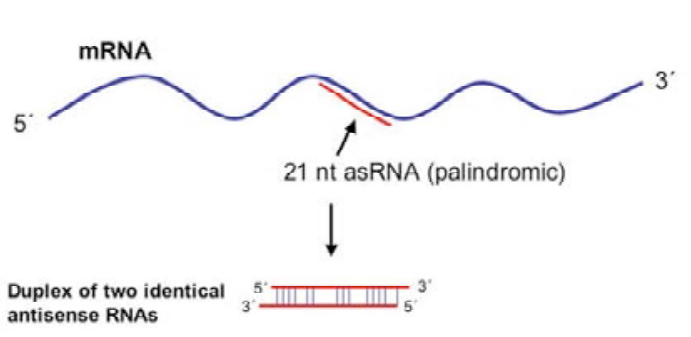

C) dual targeting siRNA

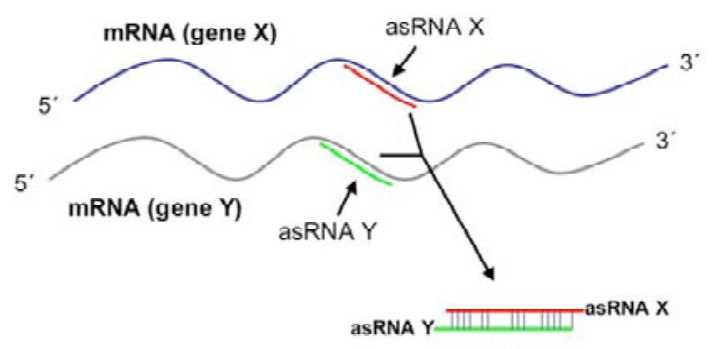

B) two target site siRNA

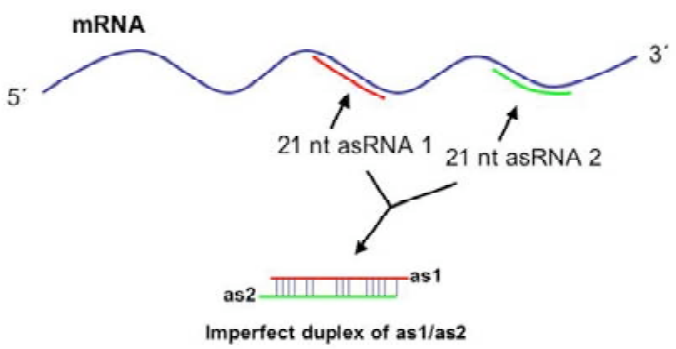

Figure 18: Strategies for rational siRNA design. The palindromic approach is shown in (A). A near palindromic target region is defined the target mRNA (blue) and the corresponding antisense oligonucleotide is synthesized (red). The antisense strand can form an RNA/RNA duplex and be used as an siRNA. Two target regions (red and green), complementary to each other, are defined on a single target mRNA (blue). For the two target site approach both antisense oligonucleotides can be annealed and the RNA duplex is used as an siRNA. The dual targeting (C) is very 
similar to the two target site siRNA, but here the antisense oligonucleotides have their target regions on two different mRNAs (blue and grey).

\section{III.3.1 Silencing of lamin A/C with self complementary asRNAs}

A set of different self complementary regions in the lamin $\mathrm{A} / \mathrm{C}$ cDNA sequence were identified and were used to design and synthesis of siRNAs. Sense and antisense RNAs were synthesized for the positions starting from positions 16, 94, 447 and again 608 relative to the first nucleotide of the start codon. All siRNAs were $5^{\prime}$-phosphorylated single strands. SiRNA sequences and predicted structures of the as/as siRNAs can be found in Table 4. The siRNAs were annealed either as s/as siRNA or as as/as siRNA and transfected into human HeLa SS6 cells. Indirect immunofluorescence with the lamin A/C specific antibody 44 hours after transfection revealed a decrease in lamin $\mathrm{A} / \mathrm{C}$ expression. By this assay all s/as siRNAs and three out of the four as/as siRNAs were efficient in silencing the lamin A/C gene (Fig. 19).

Table 4: Sequences and structures of palindromes.

\begin{tabular}{|c|c|c|c|c|}
\hline Pos & s/as siRNA & & as/as siRNA & \\
\hline 608 & $\begin{array}{l}\text { CTGGACTTCCAGAAGAACATT } \\
|||||||||||||||||| \\
\text { TTGACCTGAAGGTCTTCTTGT }\end{array}$ & $\begin{array}{l}\mathrm{S} \\
+++ \\
\text { as }\end{array}$ & $\begin{array}{l}\text { CTGTTCTTCTGGAAGTCCAGTT } \\
\text { |||: : |||l: : |||l: : ||| } \\
\text { TTGACCTGAAGGTCTTCTTGTC }\end{array}$ & $\begin{array}{l}\text { as } \\
+++ \\
\text { as }\end{array}$ \\
\hline 94 & 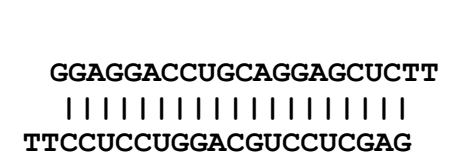 & $\begin{array}{l}\text { S } \\
+++ \\
\text { as }\end{array}$ & $\begin{array}{l}\text { GGAGCUCCUGCAGGUCCUCCTT } \\
\text { ||||: }||||||||::|||| \\
\text { TTCCUCCUGGACGUCCUCGAGG }\end{array}$ & $\begin{array}{l}\text { as } \\
- \\
\text { as }\end{array}$ \\
\hline 16 & $\begin{array}{l}\text { GCGGCGCGCCACCCGCAGCTT } \\
|\||\||\||\||\||\|\|\|\||\| \\
\text { TTCGCCGCGCGGUGGGCGUCG }\end{array}$ & $\begin{array}{l}S \\
++ \\
\text { as }\end{array}$ & $\begin{array}{l}\text { GCUGCGGGUGGCGCGCCGCTT } \\
||:|||:| .: 1:||||:|| \\
\text { TTCGCCGCGCGGUGGGCGUCG }\end{array}$ & $\begin{array}{l}\text { as } \\
+ \\
\text { as }\end{array}$ \\
\hline 447 & 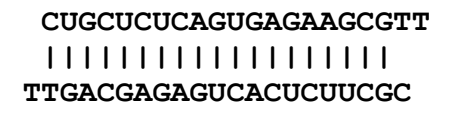 & $\begin{array}{l}S \\
++ \\
\text { as }\end{array}$ & 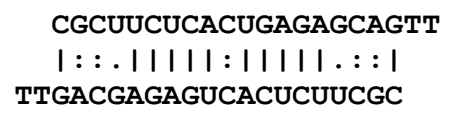 & $\begin{array}{l}\text { as } \\
+++ \\
\text { as }\end{array}$ \\
\hline
\end{tabular}


( $\mathrm{s}=$ sense, $a s=$ antisense, pos. $=$ position on lamin A/C mRNA, $+++=>70 \%$ knockdown, $++=50-70 \%,+=30-50 \%,-=0-30 \%$ )

Silencing of lamin A/C was nearly equally efficient when using siRNA 608 as either a s/as or as a as/as duplex. The same result was observed after transfection of s/as or as/as siRNAs targeting positions 447 to 468 and 16 to 37 . For target position 94 the s/as siRNA was far more efficient while transfection of the as/as duplex did not lead to a reduction of lamin A/C.

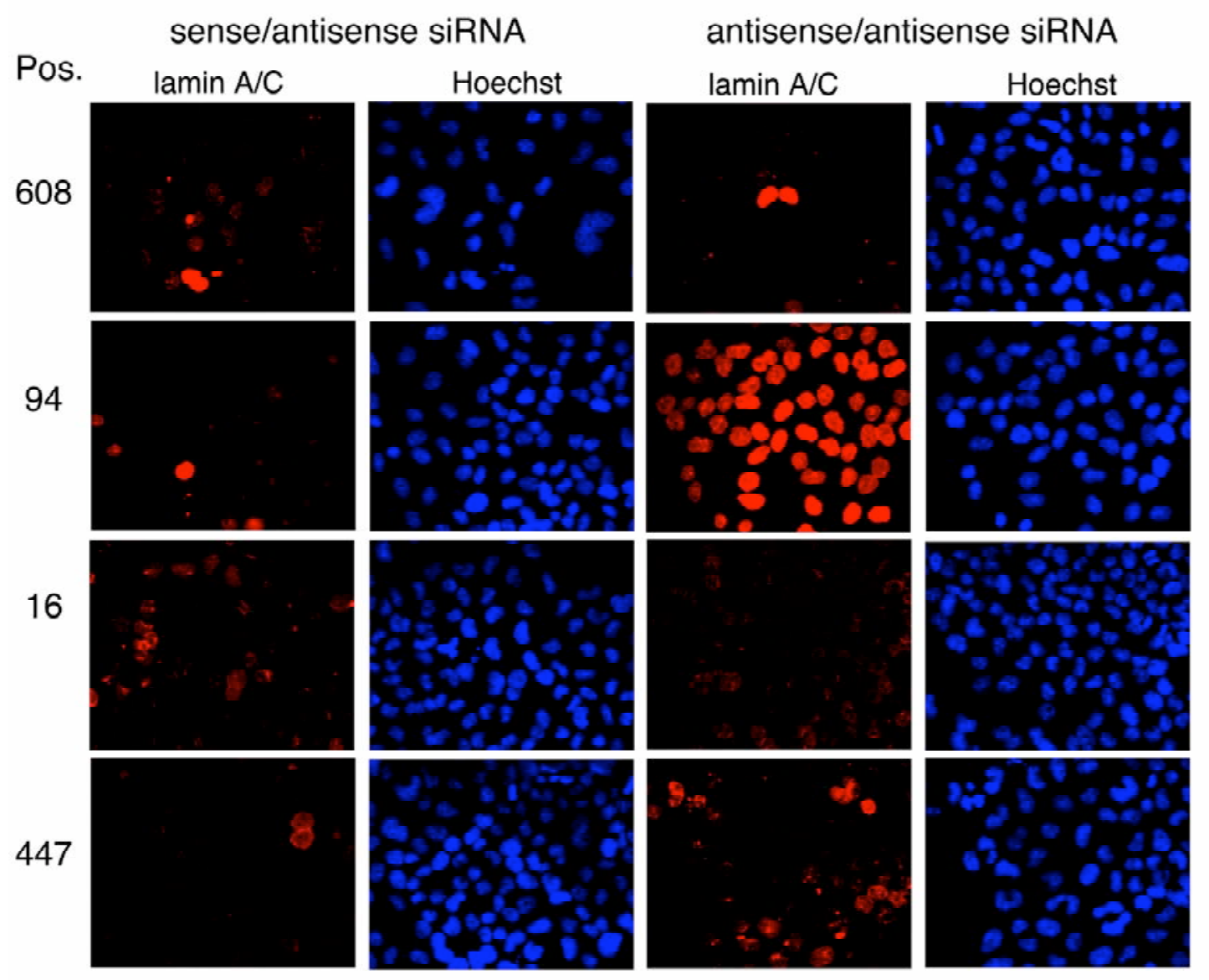

Figure 19: Indirect immunofluorescence micrographs of HeLa cells after transfection of s/as and as/as siRNAs. Cells were stained with the lamin A/C antibody 44 hours after transfection of siRNAs. DNA was visualized with Hoechst 33342. Positions of siRNA on the lamin A/C mRNA are given on the left side. The left panel shows cells transfected with s/as siRNAs, the right panel shows cells transfected with the corresponding as/as siRNAs. Both s/as and as/as siRNAs were effiecient for silencing at the positions 608, 16 and 447. The siRNA tageting position 
94 worked only as the s/as duplex, while the as/as siRNA did not silence lamin A/C. (magnification $\mathrm{x} 400$ ).

The observations from indirect immunofluorescence microscopy were confirmed by western blotting with extracts of transfected HeLa cells (figure 20). Cell extracts were subbjected to SDS-PAGE, blotted onto a nitrocellulose membrane and developed with antibodies recognizing lamin $\mathrm{A} / \mathrm{C}$ and vimentin. The vimentin antibody allowed confirmation of equal protein loading and was also used to normalize the data obtained in quantitative western blots (figure 21). Western blot analysis indicated the same silencing efficiencies as observed in immunofluorescence. The target positions 608, 16 and 447 displayed comparable knockdown levels for both s/as and as/as siRNAs. Only the as/as siRNA targeting position 94 did not lead to a significant reduction of lamin A/C.

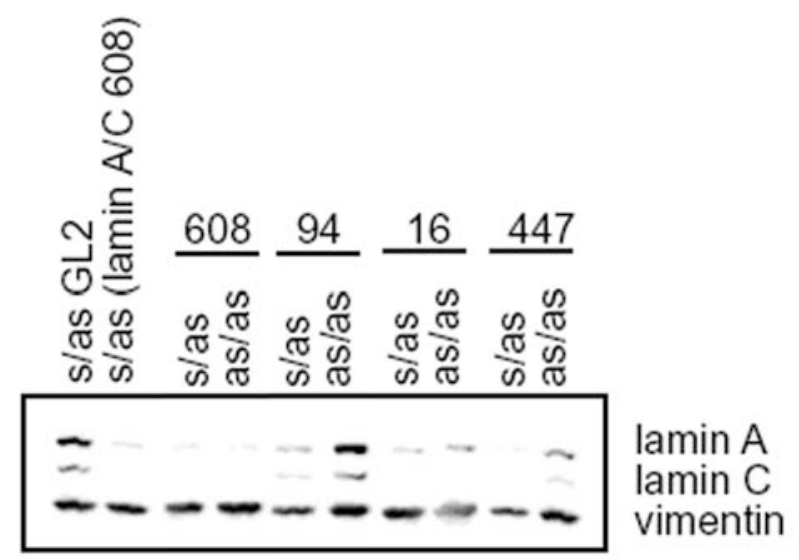

Figure 20: Western blot analysis of HeLa cells after transfection with palindromic siRNAs. Cell extracts were harvested and blotted with antibodies recognizing lamin A/C (antibody 636.23) and vimentin (antibody V9) 44 hours after siRNA transfection. The unspecific GL2 siRNA served as a negative control and the unphosphorylated lamin $\mathrm{A} / \mathrm{C}$ siRNA as a positive control. Reduction of lamin $\mathrm{A} / \mathrm{C}$ was observed for transfection of both s/as and as/as siRNAs targeting the regions 608, 16 and 447. Also transfection of the s/as siRNA 94 led to efficient knockdown, but the corresponding as/as siRNA did not show an effect. 
Western blots were also performed to obtain quantitative knockdown analysis. The emitted light was quantified by a luminescence reader (LumiImager) after ECL development (figure 21). Vimentin signals were used for normalisation and the values obtained were standardized with the GL2 siRNA negative control. Quantitative analysis revealed that silencing efficiencies of the siRNAs targeting positions 608 and 16 are equal for both s/as and as/as siRNAs. For target position 447 the as/as duplex was even more efficient than was the corresponding s/as siRNA. The as/as duplex for position 94 did not cause a knockdown of lamin A/C.

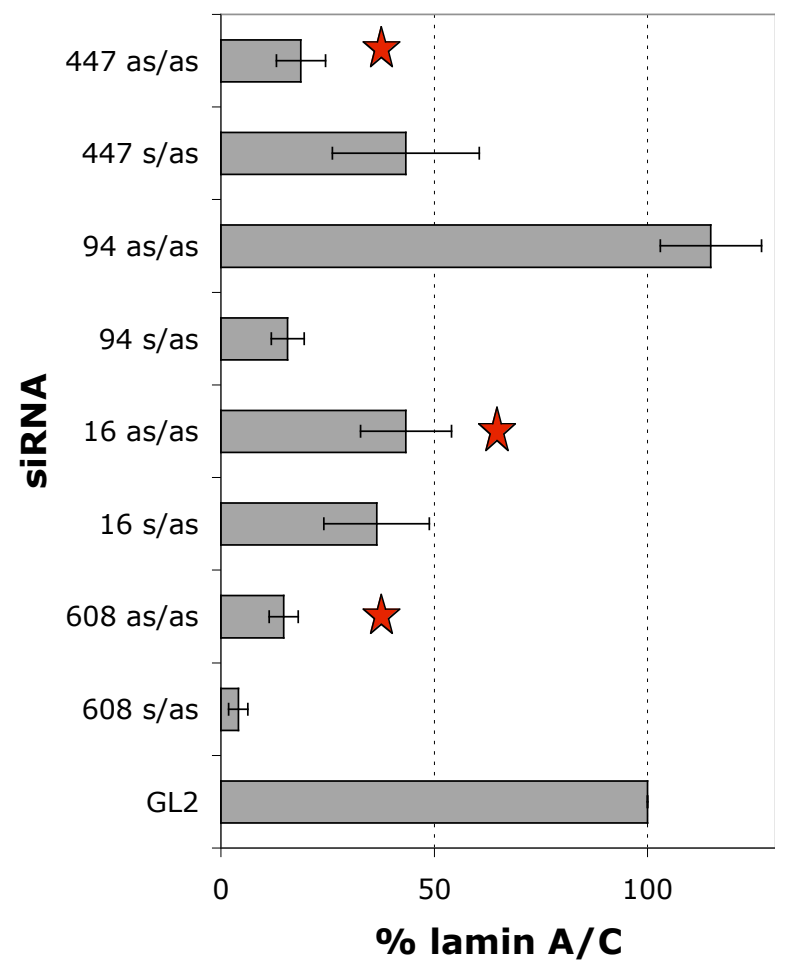

Figure 21: Quantitative western blot analysis of HeLa cell extracts after transfection of s/as and as/as siRNAs. The blots were probed with lamin A/C and vimentin antibodies and examined by a luminescence reader. Values shown are standardized with vimentin and normalized with the GL2 negative control set to $100 \%$. Functional as/as siRNAs are marked with red stars. 


\section{III.3.2 Two target site siRNAs}

After successful design and application of palindromic siRNAs to target endogenous lamin $\mathrm{A} / \mathrm{C}$ an attempt was made to find complementary target regions within the cDNA sequence. This approach should allow direct incorporation of the desired target specific antisense strands into RISC. Two pairs of complementary regions were used for the design of antisense/antisense duplexes which were then transfected into HeLa cells. Transfections of the corresponding sense/antisense siRNAs and the single antisense strands served as an internal control. Both as/as siRNAs with two target sites led to an efficient knockdown of the target gene. The levels of silencing were fully comparable to those obtained after transfection of the corrresponding s/as siRNAs. The antisense strands were not palindromic but only complementary to the second antisense strand of the pair. These antisense strands did not function as efficient gene silencers when they were transfected as ssRNAs. The immunofluorescence micrographs in figure 22 indicate the function of one as/as SiRNA. 

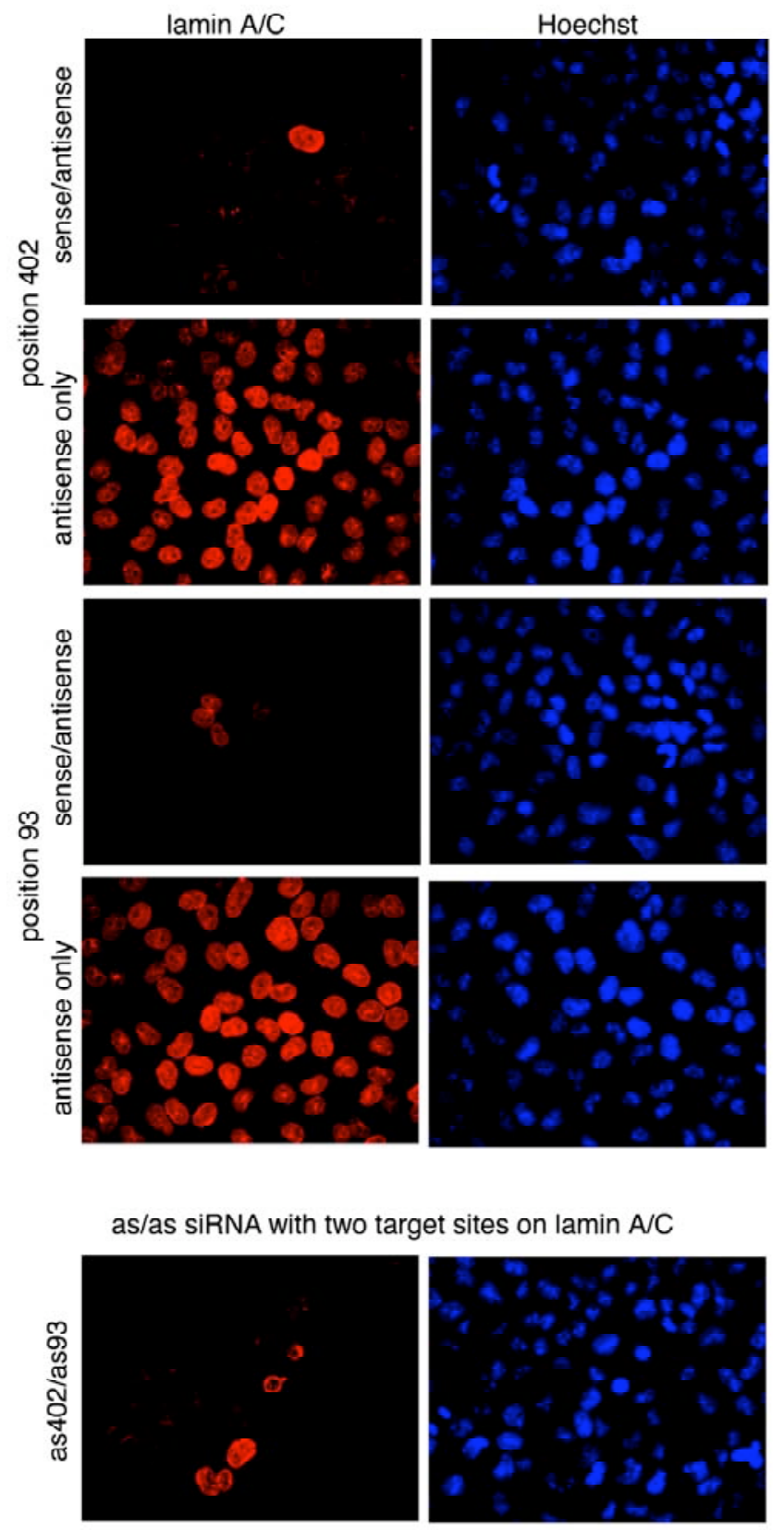

Figure 22: SiRNA with two target sites leads to knockdown of lamin $A / C$ in HeLa cells. Indirect immunofluorescence micrographs were taken 44 hours after transfection of siRNAs. A pair of antisense-complementary siRNAs against lamin $\mathrm{A} / \mathrm{C}$ was. The s/as siRNA against the first target region, i.e. 402 (top), led to lamin A/C knockdown, as well as the second s/as siRNA targeting position 93. Transfection of the antisense strands alone did not show any clear effects on lamin A/C. The imperfect duplex of the two antisense strands was efficient for silencing (bottom). DNA was visualized with Hoechst dye. (Magnification x400)

Data obtained from indirect immunofluorescence microscopy were confirmed by quantitative western blot analysis with lamin $\mathrm{A} / \mathrm{C}$ and vimentin antibodies (figure fish6) as described above. Both s/as targeting positions 402 (>80\% reduction) and 93 
(60\% reduction), significantly reduced the lamin A/C levels. A strong reduction was also observed after transfection of the as/as duplex ( $>75 \%$ reduction). Surprisingly transfection of the antisense ssRNA also led to a silencing effect. The observed knockdown was weaker ( $40 \%$ for $a s 402,45 \%$ for as 93 ) but detectable. The possibility of duplex formation will be discussed.

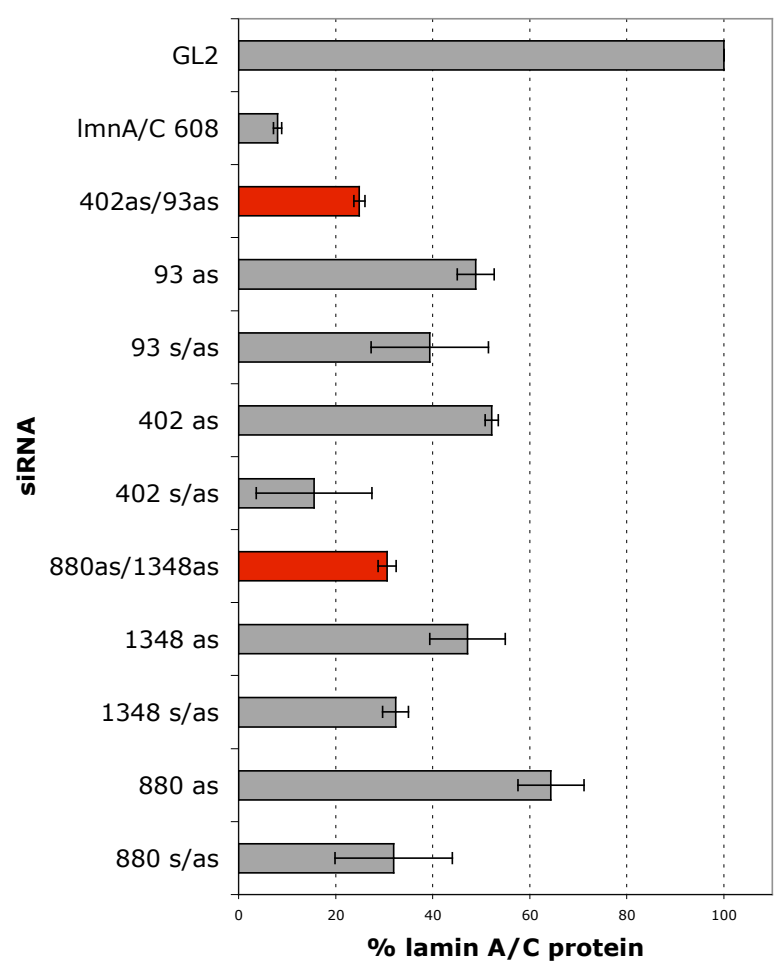

Figure 23: Quantitative western blot analysis. Two pairs of siRNAs targeting complementary target regions were used for design of siRNAs targeting positions 402 and 93 (pair 1) and 880 and 1348 (pair 2). All four s/as siRNAs efficiently silenced lamin A/C, while transfection of antisense ssRNAs only weakly reduced lamin A/C. Both duplexes of complementary antisense strands (shown in red) knocked down lamin A/C. Bars represent lamin A/C protein levels as normalized to the GL2 control.

A second pair of complementary target regions on lamin $\mathrm{A} / \mathrm{C}$ was from positions 880 and 1348. Both s/as duplexes reduced lamin $\mathrm{A} / \mathrm{C}$ by $70 \%$ after transfection into HeLa cells. The imperfect duplex of the two antisense strands knocked down lamin $\mathrm{A} / \mathrm{C}$ to $30 \%$ and thus was at least as efficient as the classical SiRNA. 


\section{III.3.3 Dual targeting siRNAs}

Since the design of two target site siRNAs was successfully used to create siRNAs which can be fully integrated into RISC, the idea was developed to select complementary regions on two different target mRNAs for siRNA design. Lamin $\mathrm{A} / \mathrm{C}$ and the lamin A interacting protein emerin were chosen, since both genes were well characterized, antibodies were available and they were known to be nonessential when silenced.

Immunofluorescence micrographs in figure 24 show the effects on HeLa cells after transfection of either the siRNAs ( $/$ as) targeting lamin $\mathrm{A} / \mathrm{C}$ or emerin or the effects of the imperfect RNA duplex formed by the two antisense strands. Immunofluorescence with antibodies recognizing lamin $\mathrm{A} / \mathrm{C}$ or emerin are shown for all individual siRNAs. Knockdown of lamin A/C and emerin with classical siRNAs and transfection of the GL2 siRNA were used as controls.

The siRNA (s/as) targeting lamin $\mathrm{A} / \mathrm{C}$ at position 778 led to a strong reduction of lamin $\mathrm{A} / \mathrm{C}$ and to mislocalization of emerin. Instead of being present in the nuclear envelope it was found in the cytoplasm. Emerin could be knocked down with the siRNA (s/as) targeting position 628 . Lamin A/C was not influenced by silencing of emerin. 
A)
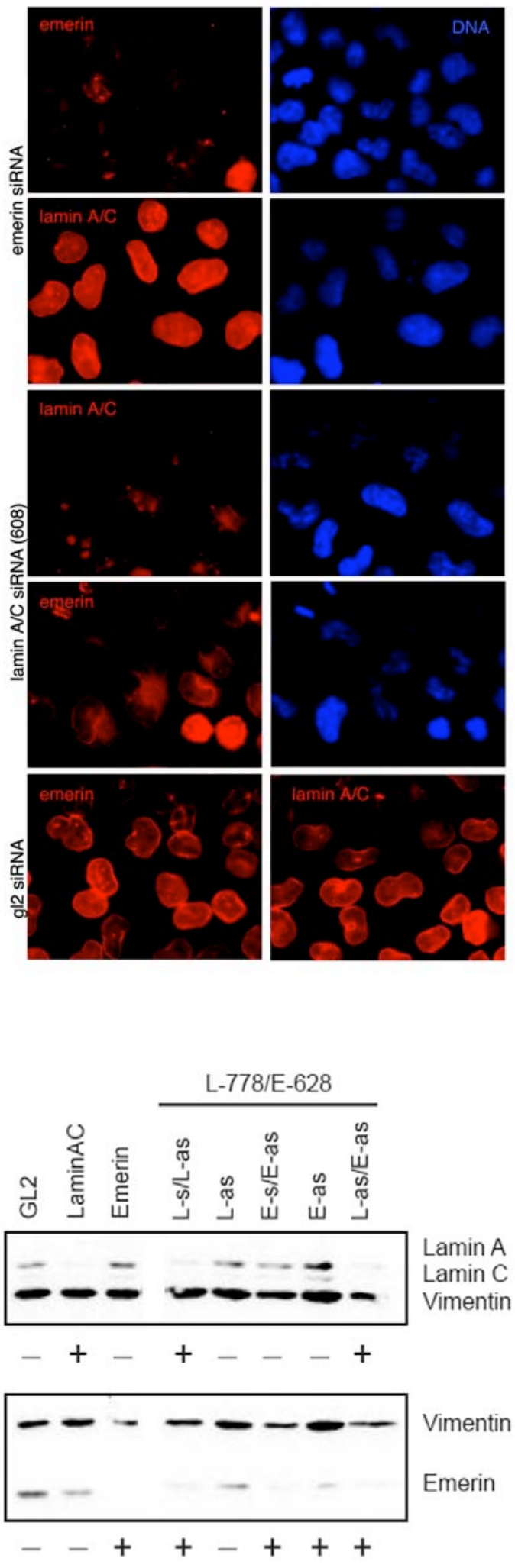

B) dual targeting siRNAs
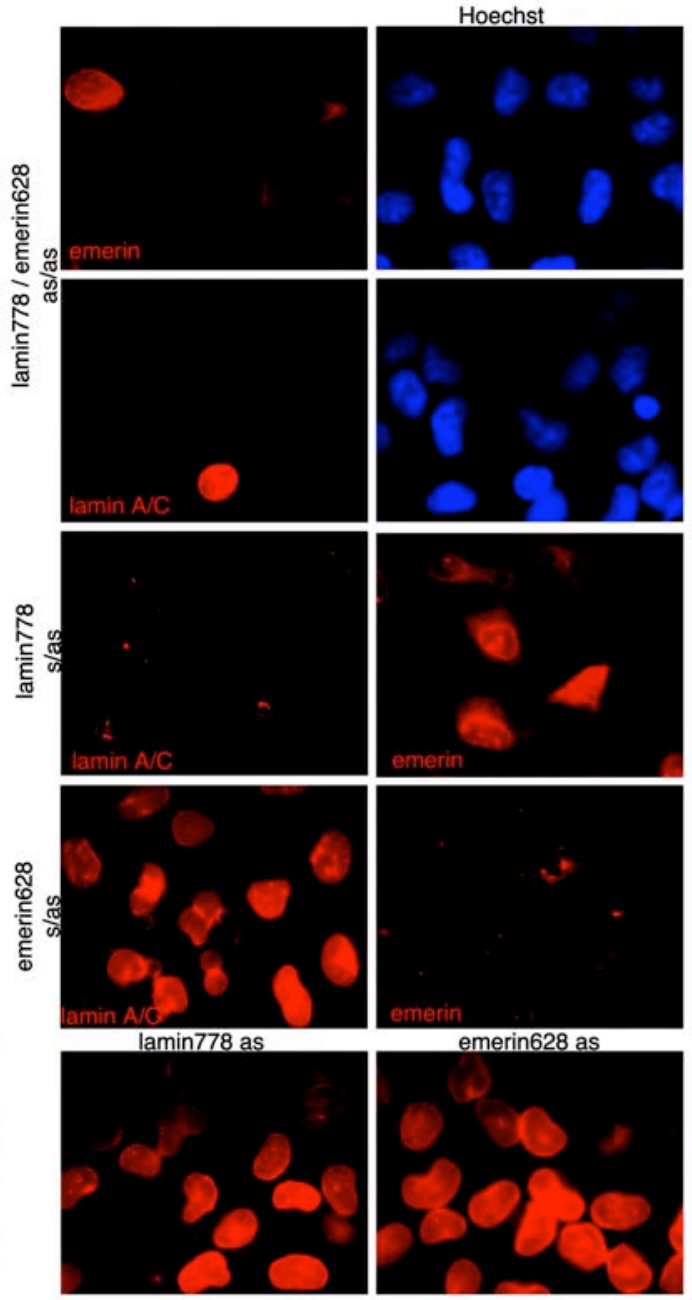

Figure 24: Silencing of two targets with a single siRNA. SiRNAs with complementary target sites on lamin A/C and emerin were transfected into HeLa cells. The imperfect dsRNA consisting of the two antisense strands (as778/as628) was functional for silencing of both targets. Cells were stained with emerin and lamin $\mathrm{A} / \mathrm{C}$ antibodies. Magnification $\mathrm{x} 480$ )

Data were confirmed by western blot analysis from siRNA transfected cell extracts.

For details see text.

The anisense strands of the siRNAs lamin778 and emerin628 formed a relatively stable duplex and were transfected in this form into HeLa cells. A strong 
reduction of both lamin $\mathrm{A} / \mathrm{C}$ and emerin was observed. Transfection of the individual antisense RNAs alone did not cause significant effects on the levels of either of the proteins.

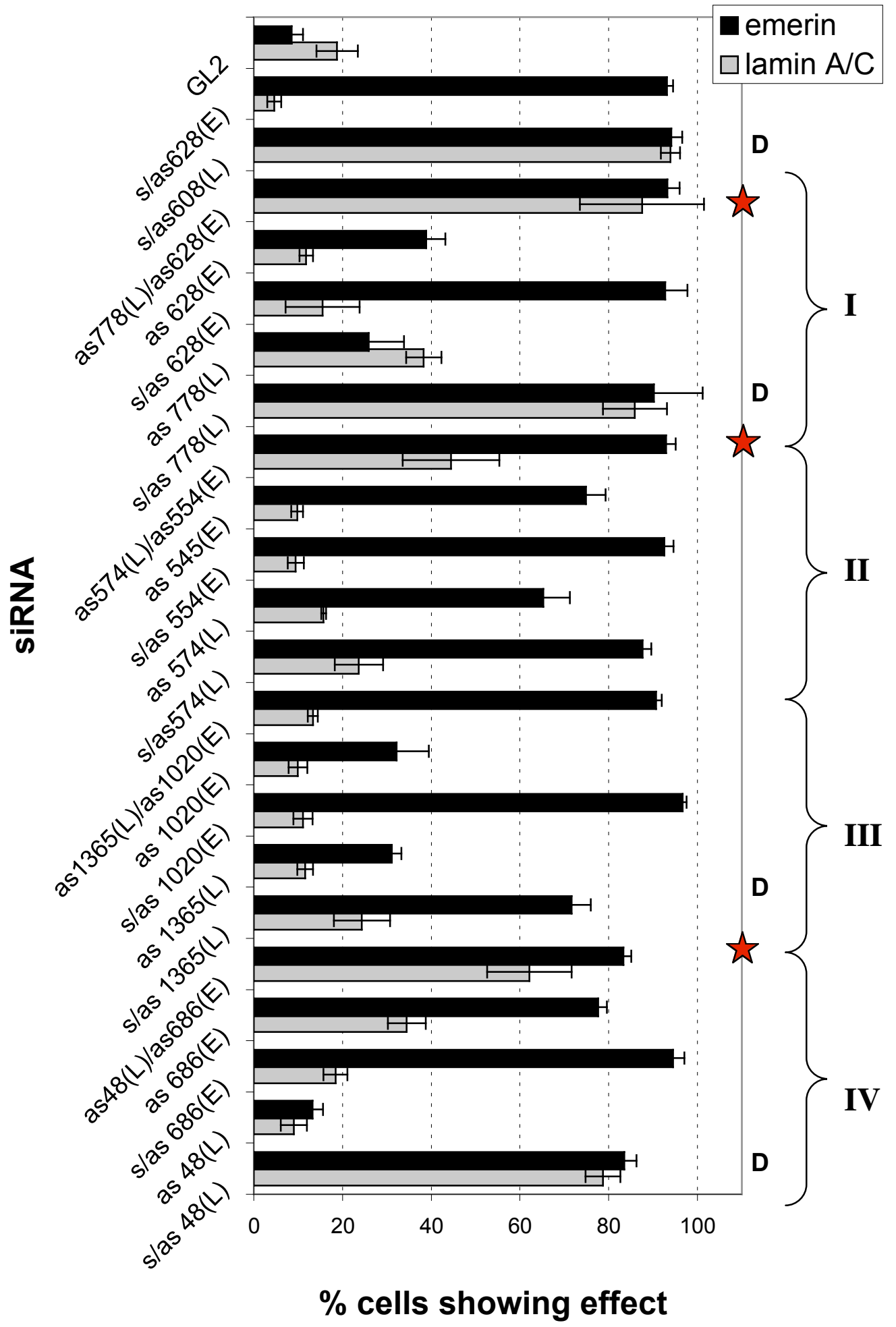


Figure 25: Dual targeting siRNAs in quantitative analysis. siRNAs were transiently transfected into Hela SS6 cells, and expression of emerin and lamin A/C was analyzed after 48 hours by quantitative immunofluorescence microscopy. siRNA duplexes against emerin (E) and lamin A/C (A) and dual target duplexes against emerin and lamin A/C were tested for RNAi efficacy. Red stars indicate functional dual targeting with antisense heteroduplexes. Emerin displacement is indicated by a D at appropriate positions. Each experiment was normalized to GL2 siRNA (background substraction). Note that bars represent numbers of affected cells, showing decreased levels of the individual target or displacement of emerin. In contrast to other graphs in this section longer bars mean better silencing.

\section{III.3.4 $\underline{\text { SiRNA rescue }}$}

According to recent results concerning the ideal design of siRNAs (see introduction) the efficiency depends on bond energies of the basepairs in the $3^{\prime}$ and $5^{\prime}$ ends of the siRNA. The helicase that unwinds the siRNA attacks preferentially the side with the lower energy. Therefore A-U bonds on the $5^{\prime}$ end of the antisense strand are postulated to increase the efficiency of an siRNAs. To experimentally proove this result two inefficient siRNAs targeting lamin $\mathrm{A} / \mathrm{C}$ were chosen. Both of them were used in previous experiments and did not reduce the target by more than $20 \%$.

The sequences of both antisense strands contained a $\mathrm{G}$ in the $5^{\prime}$-end, and thus the strongest possible basepair. For sequences and structures of siRNAs see figure lamin9. To increase the efficiency of the siRNAs the Cs at position 19 of the sense strand were replaced by an $\mathrm{A}(\mathrm{s} 1150)$ or an $\mathrm{U}$ (s994). These modifications dramatically increased the functionality of the siRNAs as assayed with quantitative western blots. Both siRNAs with mismatching sense strand reduced lamin A/C expression by more than 50\%. Additionally A-A (s1150) and G-G (s994) mismatches were introduced to destabilize the $5^{\prime}$ antisense region of the siRNAs. By adding these mismatches the efficiency of both siRNAs coul be further increased by 
some $10 \%$. Thus, destabilisation of the helicase attack end of the siRNA may be a mechanism by which poor siRNAs may be turned into highly efficient siRNAs.

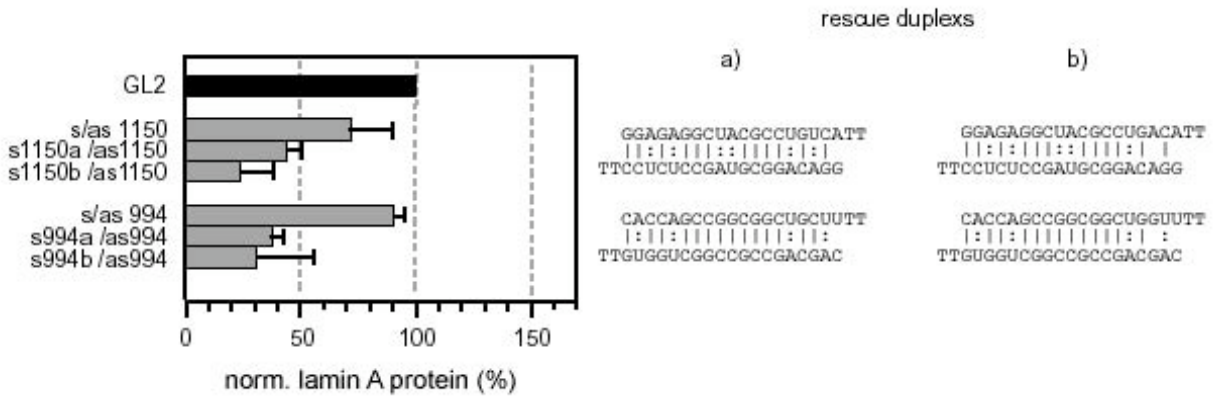

Figure 26: SiRNA rescue by destabilisation increases silencing efficiency. Two non-function siRNAs targeting lamin A/C (1150 and 994) reaching maximum silencing efficiencies of $<20 \%$ were modified on position 19 of the sense strand, or both on positions 17 and 19, respectively. A C to A base exchange at position 19 of the 1150 sense strand (structure a, top) increased the silencing efficiency to $>50 \%$. The same knockdown levels was achieved with a $\mathrm{G}$ to $\mathrm{U}$ exchange at position 19 of sense994 (structure a, bottom). Further base exchanges on position 17 of the sense strand (U to A on sense1150, C to $\mathrm{G}$ on sense994) increased the efficiency of both siRNAs to $>70 \%$ as determined by quantitative western blot analysis.

III.3.6 Overview of results form anatomy of siRNAs

Silencing of lamin $A / C$ with antisense homodimers against palindromic regions was successful.

Three out of four antisense palindromes led to lamin A/C knockdown efficiencies that were comparable to those obtained with classical s/as siRNAs.

Heteroduplexes of antisense RNAs targeting lamin A/C were shown to be capable of inducing RNAi.

as/as duplexes were more efficient than individual as RNAs alone. 
The dual targeting approach with heteroduplexes of antisense RNAs targeting two different genes (i.e. emerin and lamin A/C) led to significant reduction of both targets.

Not all dual targeting heteroduplexes worked successfully.

Modifications of the sense strand to lower the basepairing stability of the 5'end of the antisense strand dramatically increased the silencing efficiencies of siRNAs in the rescue approach. 


\section{III.4 Transfection of siRNAs by osmotic lysis of loaded pinosomes}

\section{III.4.1 Strategy and background}

A crucial step in RNAi experiments is the delivery of siRNAs into the target cells. Silencing efficiency directly depends on the success of the transfection. Various strategies were successful for siRNA delivery (see I.4) and different techniques have also been applied in this thesis. In this part I adapted the existing method of pinocytosis mediated transport of macromolecules into the cytoplasm of cultured cells to the delivery of siRNAs for functional RNAi. A novel transfection technique is provided and compared to the standard transfection with RNA/liposome complexes.

The pinocytosis cell-loading technique is based on the osmotic lysis of pinocytic vesicles, a method developed by Okada and Rechsteiner in 1982. Compounds to be loaded are mixed at high concentration with hypertonic medium, allowing the material to be carried into the cells via pinocytic vesicles. The cells are then transferred to a hypotonic medium, resulting in the release of trapped material from the pinocytic vesicles within the cells and filling the cytosol with the compound, which in the experiments described here are siRNAs for gene silencing.

\section{III.4.2 Delivery of siRNAs to cultured mammalian cells by osmotic lysis of} pinosomes

To directly demonstrate the cellular uptake of nucleic acids in cultured mammalian cells the pinocytosis assay was performed with fluorescently labelled siRNAs recognizing Eg5. HEK293 cells were treated with siRNA-containing pinocytosis hypertonic loading buffer and siRNA uptake was observed in live cells in a confocal laser scanning microscope. As can be seen from Fig. 27A almost all 
cells have taken up the fluorescently labelled siRNAs where they are found in pinosomes in the cytoplasm. Replacement of the hypertonic medium by hypotonic medium results in rupture of the majority of pinosomes and release of the DNA or siRNA into the cytoplasm (Fig. 27B).

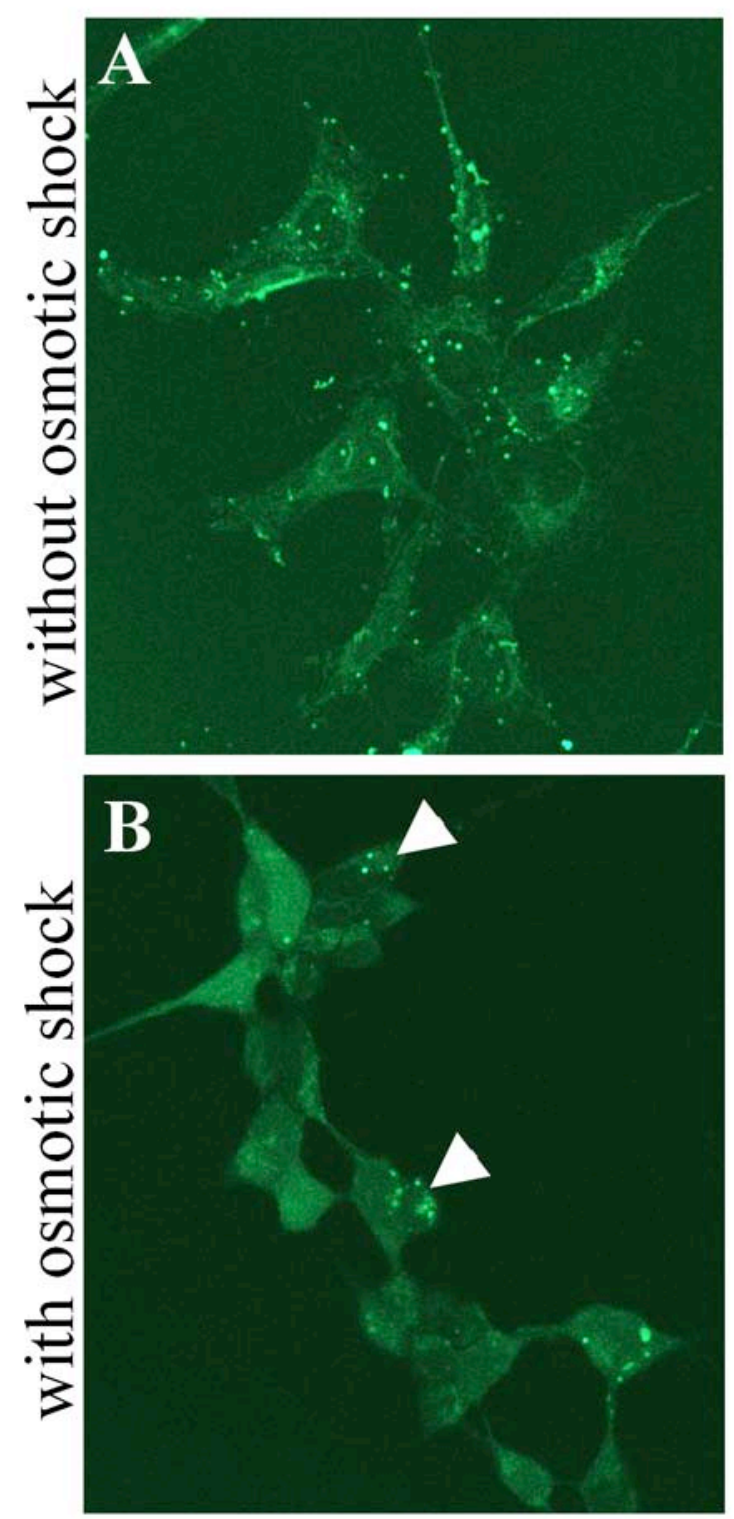

Figure 27: Direct demonstration of (A) Uptake of fluorescein-labelled siRNAs by pinocytosis in HEK293 cells and (B) release of siRNAs from pinocytotic vesicles into the cytoplasm by osmotic shock. On average, each cell has 10 to 20 fluorescent pinosomes (A). After transfer to the hypotonic medium, the majority of pinosomes are lysed although some pinosomes remain intact (arrow heads). The cytoplasmic fluorescence appears higher in (B) than in (A) suggesting that in (B) the 
osmotic lysis of pinosomes has resulted in release of the fluorescein-labelled siRNAs into the cytoplasm. Magnification x 500.

\section{III.4.3 Released siRNAs induce RNAi}

Successful uptake of siRNAs by osmotic lysis of pinosomes was also demonstrated using a morphological assay for silencing with the kinesin-like motor protein Eg5. Cells were stained with $\square$-tubulin antibody 48 hours after pinocytosis to visualize the morphological phenotype defined by monopolar mitotic spindles previously described for cells silenced with Eg5 siRNAs. Approximately $60 \%$ of the Eg5-siRNA treated cells showed the expected phenotype after 30 hours (arrow heads Fig. 28C).

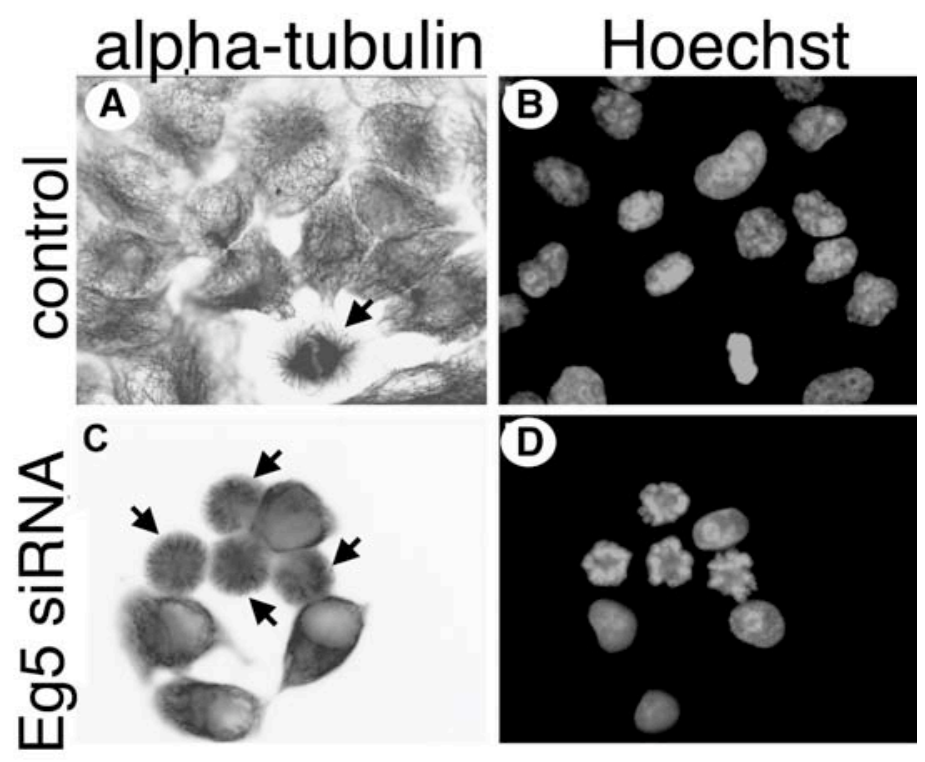

\section{no osmotic shock osmotic shock}
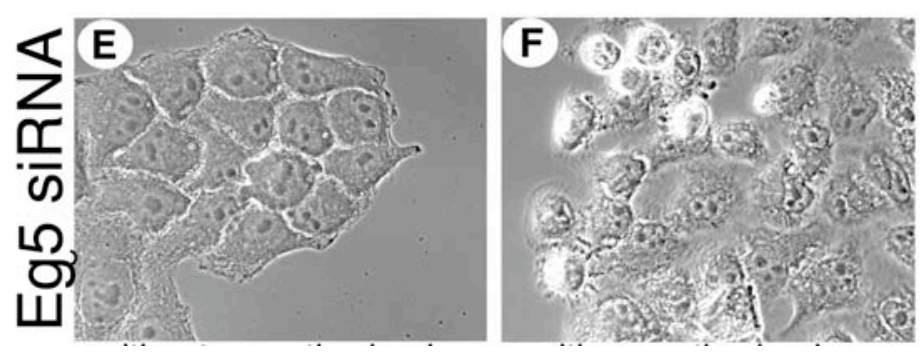

Figure 28: Pinocytosis mediated silencing of HeLa cells with siRNAs specific for the human kinesin motor protein Eg5. Cells were fixed and stained 30 hours after pinocytosis with $\square$-tubulin antibodies (A, C) and with Hoechst dye (B, D). In cells 
not exposed to siRNAs mitotic spindles when seen look normal (arrow, A). In contrast when Eg5 silenced cells are stained with $\square$-tubulin antibody many cells with monopolar spindles are apparent (arrows, C). Cells stained with $\square$-tubulin antibody $(\mathrm{A}, \mathrm{C})$ are shown in reverse contrast. $(\mathrm{E}, \mathrm{F})$ phase micrographs showing that the osmotic lysis step is required for silencing. Cells in both $(\mathrm{E})$ and $(\mathrm{F})$ were allowed to take up Eg5 siRNAs by pinocytosis. Cells in (F) but not in (E) underwent osmotic lysis of pinosomes. Note that the round cells, which if stained with tubulin antibodies display monopolar spindles are visible only in $(F)$ not $(E)$. Magnification x 480.

Eg5 siRNAs were also used to demonstrate that the hypotonic step to lyse the pinosomes is necessary (Fig. 28E, F). If this step involving exposure to hypotonic medium was omitted no rounded cells (corresponding to those shown with monopolar mitotic spindles in Fig. 28C) were detected 24 hours after pinocytosis (Fig. 28E), whereas if this step was included rounded cells were readily demonstrable (Fig. 28F).

III.4.4 Repeating the osmotic lysis of pinosomes procedure increases the silencing efficiency to that obtained with cationic liposomes

Our initial experiments with lamin A/C siRNAs showed that the efficiency of silencing by a single round of the osmotic lysis of pinosomes procedure was around $60 \%$ (Fig. 29C, D). We therefore tested whether repetition of the procedure could increase the silencing efficiency. A second procedure was performed 2, 4 or 10 hours after the first transfection using the same conditions. In the procedures in which the second step was performed 2 and 4 hours after the first treatment strongly decreased cell densities were observed (data not shown), suggesting that the cells were sensitive to a second osmotic shock if this was given within a short time interval. However, if the second pinocytotic siRNA treatment was delayed for 10 hours the 
level of silencing increased to $>90 \%$ (Fig. 29E, F) and more than $75 \%$ of the cells survived the double treatment(Fig. 29E).

The results obtained for single and double pinocytotic treatments were compared to those where lamin siRNAs were delivered by the conventional method of liposome mediated transfection using oligofectamine (Fig. 29G, H). Comparison of Fig. 29E and Fig. 29G shows that the efficiency seen with the double pinocytotic procedure is comparable to that seen with oligofectamine transfection. Efficiencies $>90 \%$ are obtainable by both methods.

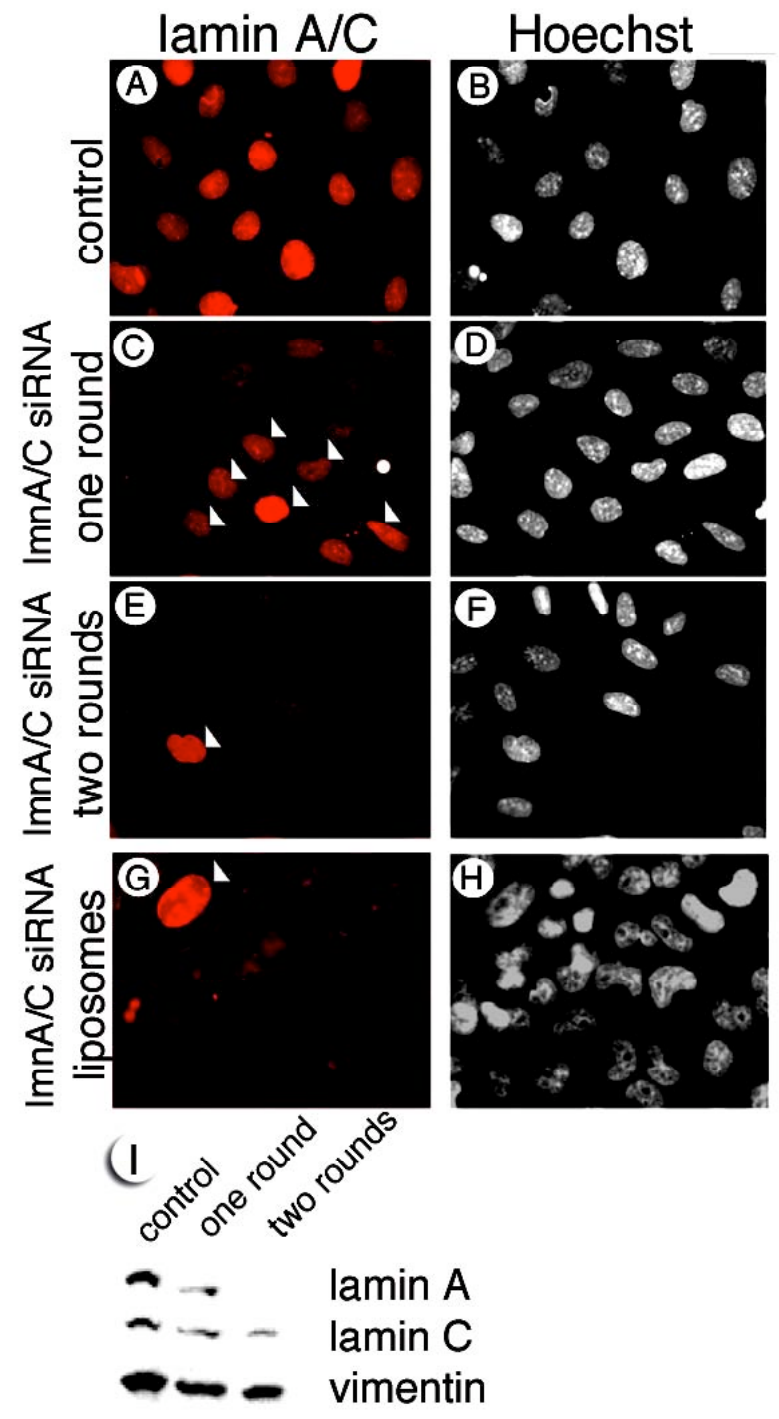

Figure 29: The silencing efficiency can be increased by using a double pinocytotic and lysis treatment. (A, B) Control Hela SS6 cells. (C, D) After a single round of treatment $\square 50 \%$ of the cells showed silencing of lamin A/C. (arrow 
headsE, F) After a double treatment $>90 \%$ of cells showed silencing of lamin A/C. Note that the cell density is somewhat decreased after the second treatment. $(\mathrm{G}, \mathrm{H})$ Liposome based transfection using lamin A/C siRNAs and oligofectamine for comparison. More than $90 \%$ of the cells show LMN A/C silencing. Arrow heads in (C, E and $\mathrm{G}$ ) show cells that are not silenced. (I) Immunoblots of HeLa cells subjected to one round of the osmotic lysis of pinosomes procedure without siRNAs (M) or to one $(1 \mathrm{x})$ or two $(2 \mathrm{x})$ rounds of treatment with siRNAs targeting lamin $\mathrm{A} / \mathrm{C}$. Magnification x 480 .

The immunofluoresence results were confirmed by Western blot analysis of cell extracts from control cells as well as with cells transfected with lamin A/C siRNAs using the pinocytotic procedure (Fig. 3I). Equal protein loading of the three samples was confirmed by probing the blots in parallel with vimentin antibody. While the amount of vimentin was equal in all three lanes the signal strength of the lamin $\mathrm{A}$ and lamin $\mathrm{C}$ bands was significantly different. In the immunoblots a reduction of $\square 50 \%$ in both lamin $\mathrm{A}$ and lamin $\mathrm{C}$ could be detected after the first round of the pinocytosis procedure with lamin A/C siRNAs. Repeating the pinocytosis after 10 hours caused a $>90 \%$ reduction in the lamin $\mathrm{A}$ and lamin $\mathrm{C}$ levels in comparison to control cells treated in the same way but without the addition of siRNAs to the hypertonic medium.

III.4.5 Increased loading times and higher siRNA concentrations increase silencing $\underline{\text { efficiencies }}$

Results of experiments in which the uptake time and siRNA concentration were varied are shown in Fig. 30. At a siRNA concentration of $0.4 \mu \mathrm{M}$, the concentration used for the experiments shown in Fig. 28 and 29, uptake is linear. An uptake time of 40 minutes resulted in silencing of lamin $\mathrm{A} / \mathrm{C}$ in $60 \%$ of the cells after 44 hours, while an uptake time of 80 minutes resulted in silencing of the lamin $\mathrm{A} / \mathrm{C}$ in $90 \%$ of the cells. Use of a concentration of $100 \mathrm{nM}$ resulted in much lower 
silencing (13\% after 80 minutes uptake; Fig. 30). Use of a concentration of $1.6 \mu \mathrm{M}$ resulted in somewhat higher levels of silencing particularly at the 20 minute time, but also resulted in a greater loss of cells from the coverslip at 44 hours post transfection.

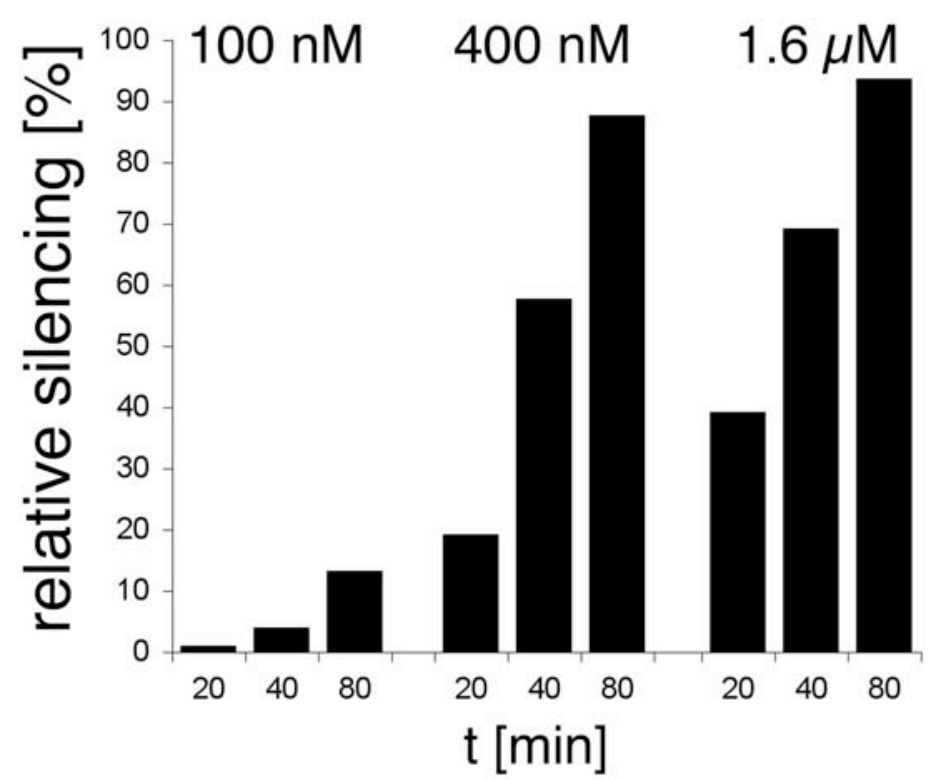

Figure 30: Uptake of siRNAs by the pinocytosis method as a function of concentration and time. HeLa SS6 cells were treated with lamin A/C siRNAs at three different concentations for 20, 40 and 80 minutes. The percentage of cells showing reduced lamin $\mathrm{A} / \mathrm{C}$ staining was measured after 44 hours.

III.4.6 Duration of transient knockdown is comparable after pinocytosis and liposome based siRNA delivery

The time course of recovery of expression of lamin $\mathrm{A} / \mathrm{C}$ after silencing by pinocytosis and by the cationic lipid method was compared (Fig. 31). Cells silenced for lamin A/C by the pinocytosis method were fully silenced at 72 hours (Fig. 31A) and by 144 hours all cells were expressing at least some lamin (Fig. 31G). Approximately half of the cells silenced by the cationic lipid method showed no 
lamin A/C expression at 144 hours (Fig. 31I). The time course of recovery is therefore faster when pinocytosis is used to get siRNAs into the cells.

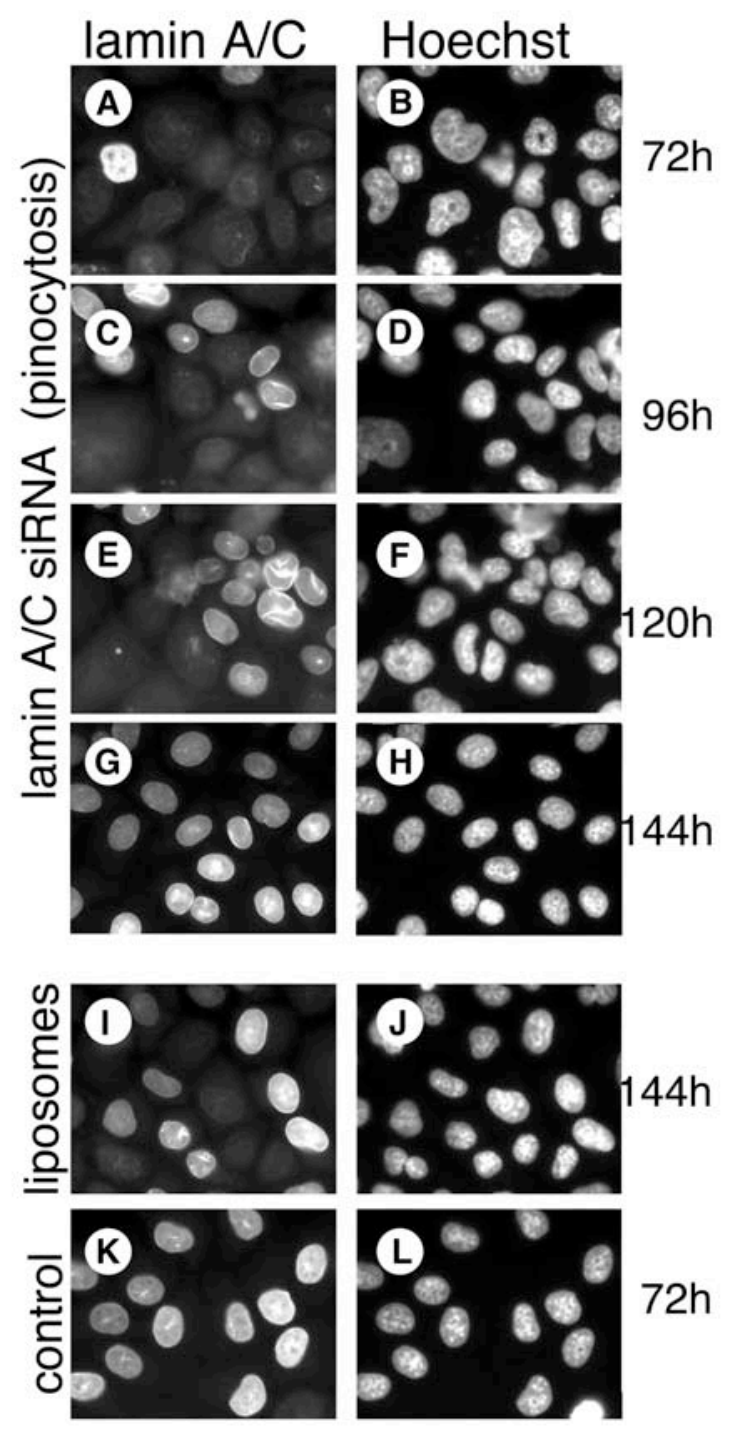

Figure 31: Comparison of time course of recovery of expression of lamin $\mathbf{A} / \mathbf{C}$ after silencing by the pinocytotic and the cationic lipid methods. Human HeLa cells (a to h) were loaded with siRNAs (1.6 $\square \mathrm{M}, 80$ minutes) targeting lamin A/C mRNA. Cells were split 54 hours after siRNA loading. The silencing effect was fully $(>90 \%)$ maintained until 72 hours after loading (a and b). Subsequently the cells started to recover expression of the lamin A/C protein until after 144 hours ( $g$ and h) all cells were lamin $\mathrm{A} / \mathrm{C}$ positive. Cells in ( $\mathrm{i}$ and $\mathrm{j}$ ) were transfected using liposomes and oligofectamine with lamin A/C siRNAs. Approximately $40 \%$ of the cells were still silenced 144 hours after transfection ( $\mathrm{i}$ and $\mathrm{j}$ ). Control cells loaded with nonspecific GL2 siRNA did not show silencing of lamin A/C ( $\mathrm{k}$ and i). (Magnification $\mathrm{x} 400)$ 
III.4.7 Pinocytosis mediated transfection is a useful method for siRNA silencing of

\section{different targets}

To check that the method also works with murine cells mouse SW3T3 cells were subjected to pinocytosis mediated transfection with siRNAs targeting the mouse lamin A/C mRNA. Delivery of siRNAs was performed with the double pinocytosis assay. Control cells were subjected to the same procedures without siRNAs (Fig. 32A, 32B). Examination of the transfected cells by indirect immunofluorescence microscopy after staining with the appropriate monoclonal lamin $\mathrm{A} / \mathrm{C}$ antibodies showed a reduction of lamin $\mathrm{A} / \mathrm{C}$ expression in $>90 \%$ of the cells (Fig. 32C, D).
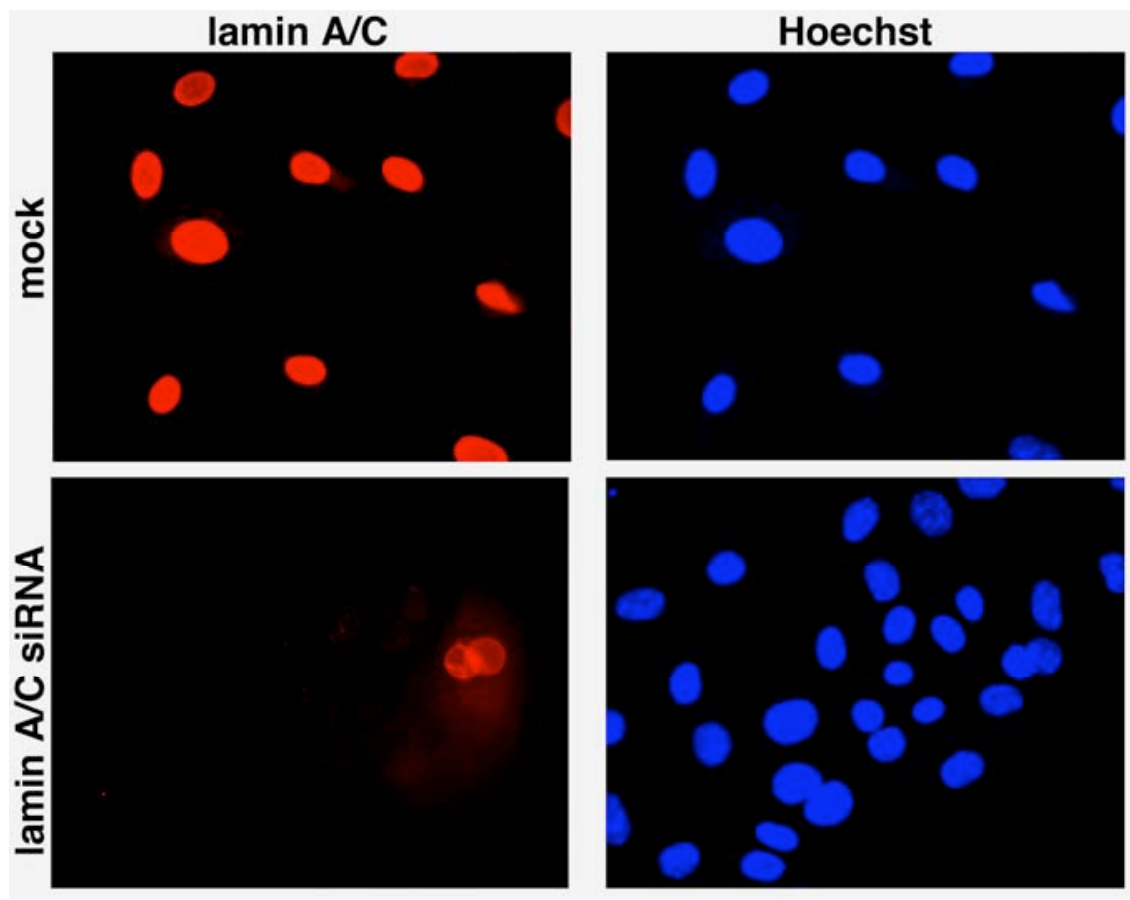

Figure 32: Gene silencing of lamin $\mathrm{A} / \mathrm{C}$ in cells of murine origin using the double pinocytosis method. Control cells subjected to the procedure without siRNAs are strongly lamin A/C positive (A, B). Exposure of mouse $3 \mathrm{~T} 3$ cells to siRNAs targeting the mouse lamin $\mathrm{A} / \mathrm{C}$ mRNA sequence resulted in lamin $\mathrm{A} / \mathrm{C}$ silencing (C, D). Arrow indicates a cell that is not silenced. Magnification x 480. 
In addition to the experiments with $\mathrm{Eg} 5$ and lamin $\mathrm{A} / \mathrm{C}$ reported above, siRNAs targeting the nuclear protein Ki67 were used. Ki67 is a useful prognostic marker for patients with a variety of different tumors. Ki67 siRNAs were introduced by the pinocytotic technique into human HeLa and HEK293 cells using the double procedure. Control cells displayed high levels of the protein in indirect immunofluorescence microscopy with the Ki67 antibody (Fig. 33A, B and E, F). Ki67 is found in almost all nuclei, where it is strongly expressed in nucleoli. Double transfection with Ki67 siRNAs led to a loss of the Ki67 protein in more than $90 \%$ of the cells 48 hours after the first pinocytotic treatment (Fig. 33C, D and 33G, H).

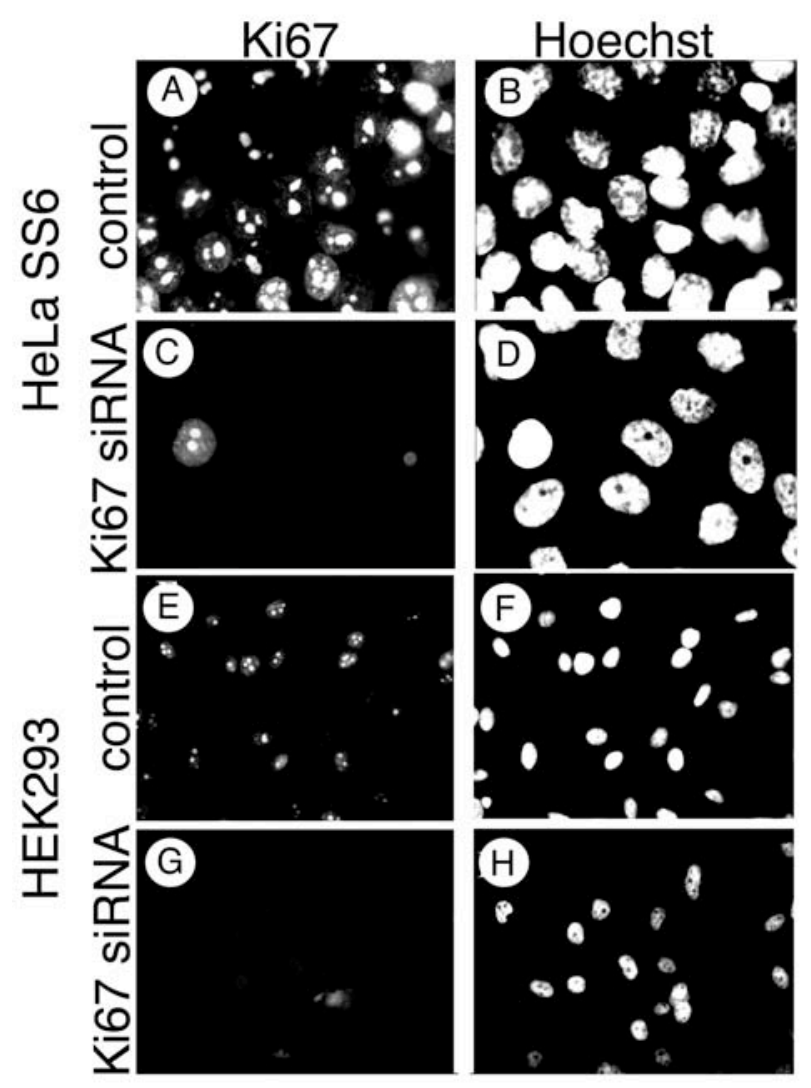

Figure 33: Gene silencing of Ki67 in human HeLa and HEK293 cells using the double pinocytosis method. HeLa cells (A-D) and HEK293 cells (E-H) were subjected to the procedure without siRNAs (A, B and E, F) or were subjected to a double pinocytosis procedure with siRNAs targeting Ki67 mRNA (C, D and G, H). Ki67 protein is present in all cells of the control populations (A, B and E, F). In 
contrast Ki67 siRNA transfected cells show silencing of the protein in the majority of cells in indirect immunofluorescence microscopy with the monoclonal Ki67 antibody ( $\mathrm{C}$ and $\mathrm{G})$. Hoechst staining is used to visualize the nuclei of all cells in the same fields (D and H). Magnification x 480 (A-D), x 300 (E-H).

III.4.8 Overview of results from pinocytosis mediated siRNA delivery

SiRNAs could be delivered into the cytoplasm of cultured cells.

Release of siRNAs by osmotic lysis pf pinosomes is necessary to induce RNAi.

Repeated cell loading, increased loading times and increased siRNA

concentration improve the silencing efficiency.

Optimized protocols allowed silencing of lamin A/C with comparable

efficiency and duration as liposome based transfection of the same siRNA.

Positive results for silencing different targets (Eg5, lamin A/C and Ki67) were

provided for different cell lines (HeLa SS6, HEK293, SW3T3) from different species

(mouse and human). 


\section{III.5 RNA interference in the zebrafish (Danio rerio)}

The strategy of this part of the thesis was to determine the capability of cultured zebrafish cells to perform RNAi. Cells were used because characterization of knockdown effects and efficiencies is well established and easier than studies on individual embryos.

III.5.1 Generation of specific siRNAs targeting exogenous and endogenous mRNAs

To test if the zebrafish can use siRNAs for functional RNAi a fusion construct for expression of fluorescent mGFP was generated. The coding sequence was cloned into a CMV-vector (provided by Dr. Erez Raz), and expression of the mGFP was under control of the strong constitutive CMV promoter. Four individual siRNAs (siGFP1-4), each targeting the MGFP mRNA were synthesized. The silencing efficiency of the siRNAs was tested in cultured human HeLa cells, either by cotransfection of plasmid and siRNA, or by sequential transfection first of the plasmid and the of the siRNA. Alternatively the plasmid and the siRNA were microinjected into HeLa cells. Fluorescence microscopy and western blot analysis of transfected HeLa cells showed that three of the GFP siRNAs (siGFP1, 2 and 4) were efficient in silencig. SiGFP1 and 2 were therefore used to silence exogenous MGFP in cultured zebrafish cells

$\square$-actin, lamin A and lamin B2 were chosen as endogenous targets. SiRNA sequences and positions were selected according to the latest known requirements for efficient siRNA design as described. For siRNA sequences and target accession numbers see Table 1.

\section{III.5.2 Silencing of exogenous mGFP in cultured zebrafish cells}

Transfection efficiencies for the two zebrafish cell lines used in these experiments were determined using classical methods of siRNA delivery. Both cell 
lines were hard to transfect, with a maximum efficiency of $35 \%$ transfected cells using cationic liposomes, and an efficiency of $<30 \%$ using optimized electroporation (Nucleofector, Amaxa). Therefore microinjection was used as a third method in silencing experiments with zebrafish cell lines. For an overview of target mRNAs, cell lines and siRNA delivery techniques see Table 5.

Table 5: RNAi in zebrafish; cell lines, targets, siRNAs

\begin{tabular}{|c|c|c|c|c|c|}
\hline $\begin{array}{l}\text { cell } \\
\text { line }\end{array}$ & origin & targets & $\begin{array}{c}\text { delivery } \\
\text { method }\end{array}$ & outcome & $\begin{array}{c}\text { RNAi } \\
\text { detection }\end{array}$ \\
\hline \multirow[t]{4}{*}{ SDJ.1 } & adult & $\operatorname{lmn} A$ & liposomes & + & IF \\
\hline & & $\operatorname{lmnB} 2$ & $\begin{array}{l}\text { liposomes } \\
\text { microinjection }\end{array}$ & $\begin{array}{c}+ \\
++\end{array}$ & IF \\
\hline & & $\square$-actin & liposomes & + & morphology \\
\hline & & pMGFP & $\begin{array}{l}\text { liposomes } \\
\text { microiniection }\end{array}$ & $\begin{array}{l}+ \\
+\end{array}$ & $\begin{array}{c}\text { GFP } \\
\text { fluorescence }\end{array}$ \\
\hline \multirow[t]{6}{*}{$\mathrm{ZFL}$} & adult & $\operatorname{lmn} A$ & liposomes & $(+)$ & IF \\
\hline & (liver & & microinjection & ++ & \\
\hline & epithelium) & $\operatorname{lmnB2}$ & microinjection & ++ & IF \\
\hline & & - -actin & liposomes & + & morphology \\
\hline & & $\begin{array}{l}\text { mGFP } \\
\text { mRNA }\end{array}$ & microinjection & $\begin{array}{c}++ \\
\text { (fluorescenc } \\
\text { e only) }\end{array}$ & n.d. \\
\hline & & $\begin{array}{l}\text { pMGFP } \\
\text { (plasmid) }\end{array}$ & microinjection & ++ & $\begin{array}{c}\text { GFP } \\
\text { fluorescence }\end{array}$ \\
\hline \multirow[t]{3}{*}{$\mathrm{ZF} 4$} & embryonic & $\operatorname{lmnB} 2$ & microinjection & ++ & IF \\
\hline & fibroblast (24h) & $\begin{array}{l}\text { MGFP } \\
\text { mRNA }\end{array}$ & microinjection & $\begin{array}{c}+ \\
\text { (fluorescenc } \\
\text { e only) }\end{array}$ & n.d. \\
\hline & & $\begin{array}{l}\text { pmGFP } \\
\text { (plasmid) }\end{array}$ & microinjection & + & $\begin{array}{c}\text { GFP } \\
\text { fluorescence }\end{array}$ \\
\hline
\end{tabular}

The adult zebrafish cell lines SJD and ZFL were transfected with the mGFP plasmid in the presence and absence of siRNAs. Up to of 35\% of the cells expressed mGFP protein in the absence of siRNA. When siRNAs specific for GFP (siGFP1 or siGFP2) were cotransfected together with the plasmid less than $5 \%$ of the cells 
showed detectable levels of mGFP in fluorescence microscopy (Figure 34). Significant effects on the viability of cells were not observed.
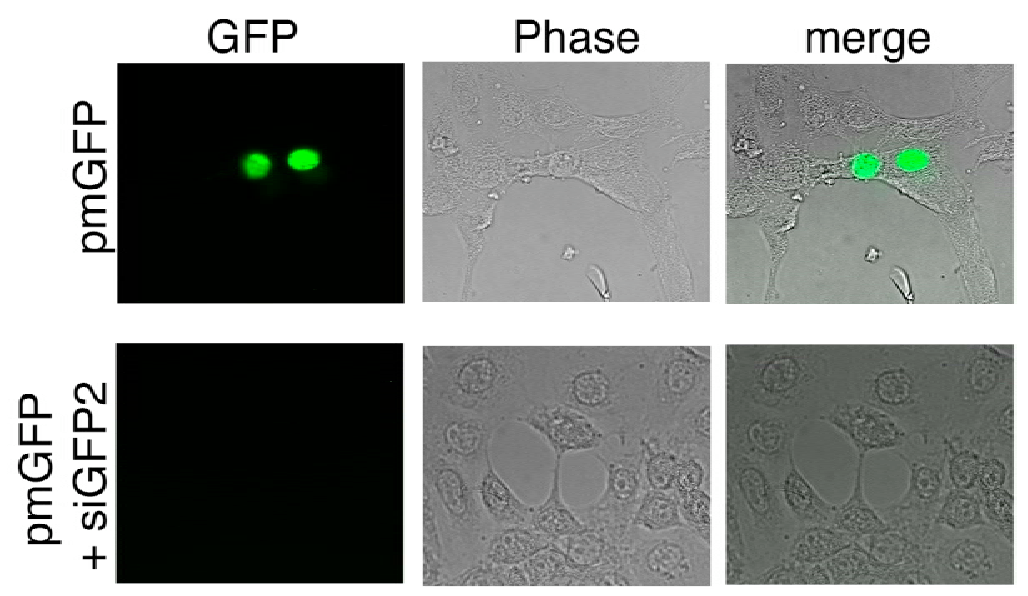

Figure 34: Silencing of exogenous GFP in zebrafish cells. ZFL cells were subjected to fluorescence microspcopy 24 hours after transfection of the GFPplasmid alone or in combination with the siGFP2 siRNA. The population transfected with the plasmid alone expressed GFP in 35\% of cells (upper panel) as observed in fluorescence microscopy. Cotransfection of siGFP2 resulted in a strongly reduced number of GFP positive cells (lower panel).

\section{III.5.3 Silencing of endogenous genes in cultured zebrafish cells of adult and} embryonic origin

Transfection of $\square$-actin siRNA resulted in morphological changes in the cells, visible as membrane blebbing and rounding of cells as previously described (Figure 35). Morphological changes result from a decrease in microfilament bundles after knockdown of $\square$-actin. This experiment also indicated that siRNA per se is not responsible for unspecific effects, since cells treared with the unspecific GL2 siRNA (siGL2) did not cause alterations in cell growth or morphology. 


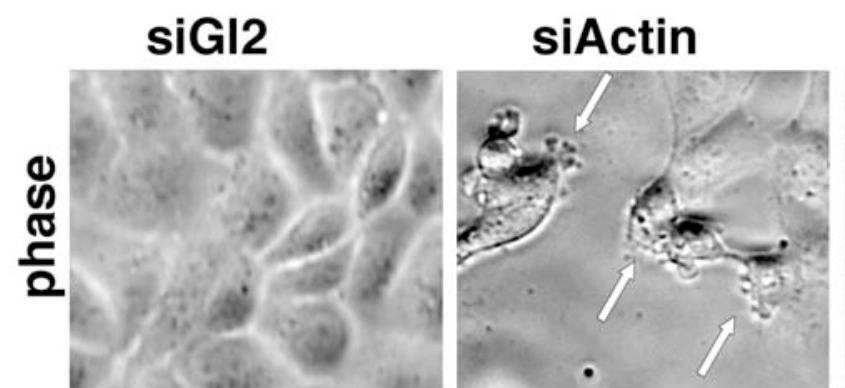

Nomarski

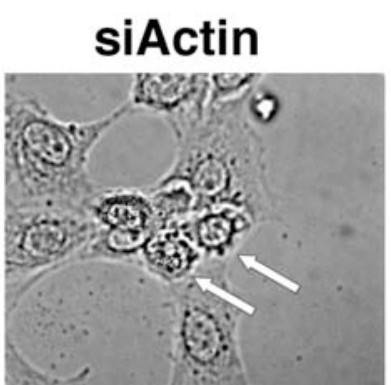

Phase

Figure 35: ZFL cells show morphological changes after transfection of $\square$-actin siRNA. Cell transfected with control siRNA (siG12) showed the normal morphology. Transfection of $\square$-actin siRNA resulted in morphological changes in $30 \%$ of the cells 44 hours after transfection. Cells silenced for $\square$-actin round up and display typical membrane blebbing (white arrows).

After succesful silencing of $\square$-actin in adult zebrafish cells in culture we selected lamin A as the next target. Lamin A is expected to be non-essential for the zebrafish cells and silencing of lamin A in cultured cells of other species (human and mouse) indicated no effects on their morphology and viability. SDJ and ZFL cells were transfected with the lamin A specific siRNA and subsequently examined by indirect immunofluorescence microscopy with lamin A antibody (Figure 36). Control cells were transfected with GL2 siRNA. A significant reduction in the amount of lamin A could be observed in $30 \%$ of the cells.

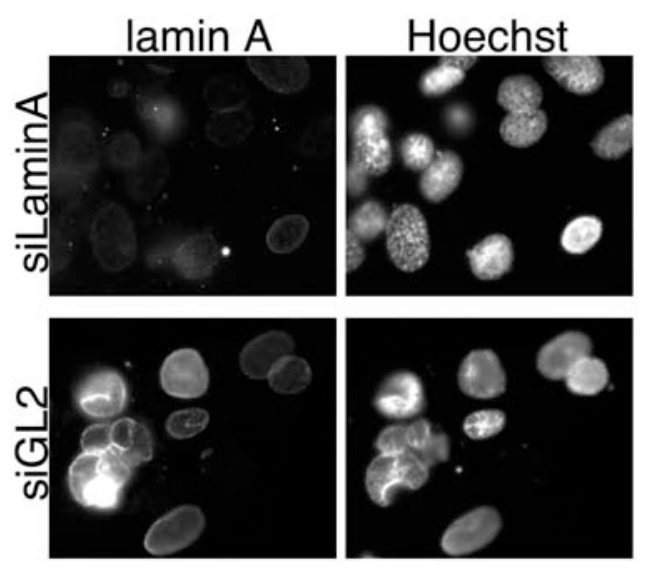

Figure 36: RNAi in cultured zebrafish cells after transfection of lamin A siRNA. ZFL cells were transfected with siRNAs using oligofectamine and examined 
by indirect immunofluorescence microscopy with specific antibodies for lamin A 44 hours after transfection. Note the reduction of lamin A protein in silenced cells (top) in comparison to GL2 siRNA transfected control cells (bottom).

To test wether the weak silencing efficiency was due to incomplete transfection or if it is a systemic problem of zebrafish cells to perform RNAi, siRNAs were delivered into the cells by microinjection. This method can be used to control the amount of siRNA that is delivered into the cell and to follow the fate of each cell at later time points. Injection of lamin A siRNA into the cytoplasm of ZFL and SDJ cells led to a nearly complete knockdown of the protein in all injected cells. Injection of control siRNA (siGL2) did not alter lamin A levels or the cell viability (figure 37).
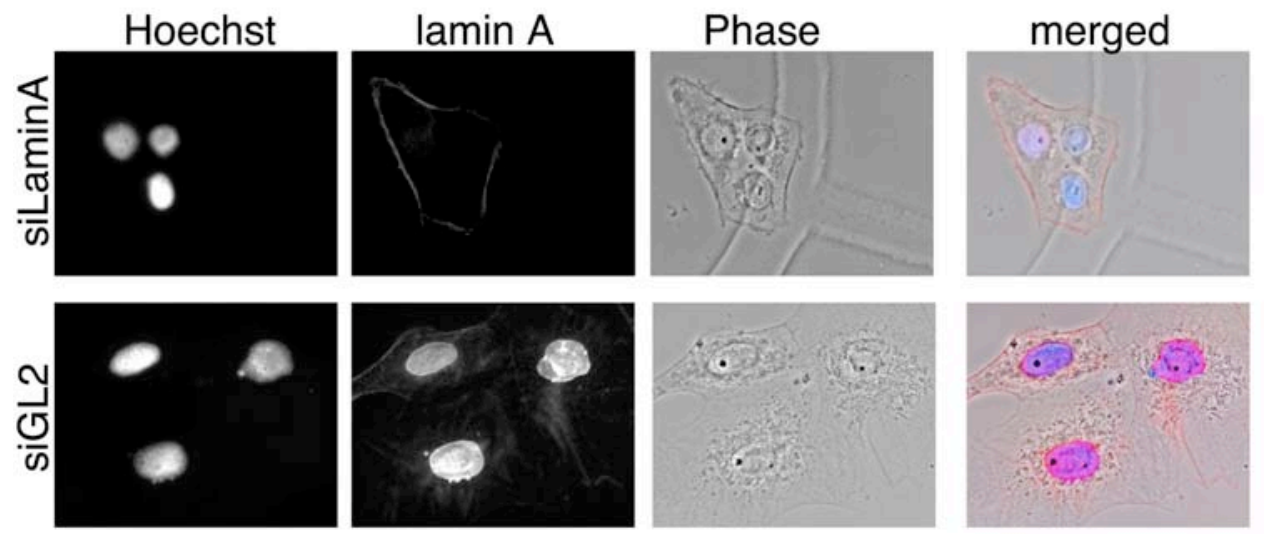

Figure 37: Microinjection of lamin A siRNA into ZFL cells resulted in knockdown of lamin A. The three cells in the top panel were microinjected with lamin A siRNA (siLaminA) and immunostained with lamin A specific antibody 40 hours after transfection. Cells display strongly reduced lamin A protein when compared to control cells (injected with siGL2) in the lower panel. DNA was visualised with Hoechst 33342 dye.

Since the two cell lines used were of adult origin a third cell line of embryonic origin was used. The ZF4 fibroblast cell line originated from 24 hours old embryos. At this developmental stage of the embryonic development lamin A is not yet expressed. ZF4 cells are lamin A negative and therefore lamin B2 was chosen as a suitable target for RNAi. Microinjection of siRNA against lamin B2 into ZF4 cells 
the protein level of the target gene was very efficiently knocked down. Indirect immunofluorescence revealed that the zebrafish cell line of embryonic origin was able to perform functional RNAi against the target lamin B2. Injection of control siRNA (siGL2) did not show any effects on treated cells (figure 38B). The functionality of siRNAs against lamin B2 was tested on ZFL and SDJ.1 cells (figure 38A) and efficient downregulation was observed (30\% knockdown in ZFL and SDJ.1 cells).

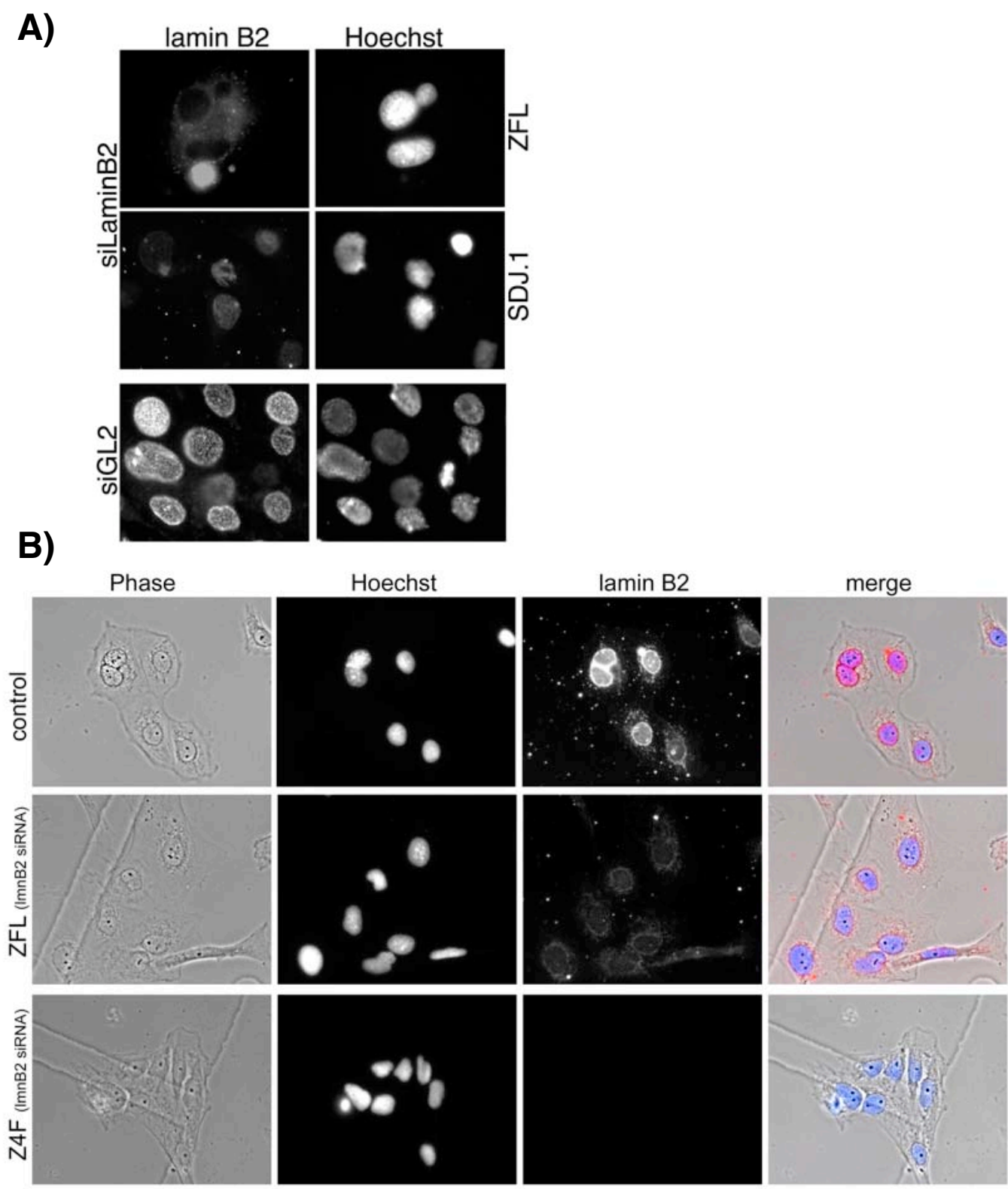

Figure 38: Silencing of lamin $B 2$ in zebrafish cells of adult and embryonic origin. SDJ.1 and ZFL cells in A were transfected with lamin B2 siRNA or with 
siGL2 as control. 40 hours after transfection cell were immunostained with lamin B2 specific antibody. Both ZFL and SDJ.1 cells display significantly reduced levels of lamin B2 protein when compared to control cells (SDJ.1 transfected with siGL2). Cells in B were microinjected with siGL2 (control, top panel) or siLaminB2 siRNAs. ZF4 cells in siGL2 injected control cells show normal levels of expression of lamin B2. ZFL cells (mid-panel) show a strong reduction in laminB2 protein levels after injection of siLaminB2. The embryonic ZF4 cells injected with siLaminB2 display a nearly complete knockdown of the lamin B2 protein.

III.5.4 Overview of results obtained with RNAi in Danio rerio

Exogenously expressed GFP was successfully silenced by sequential or cotransfection of siRNAs against GFP in cultured zebrafish cells.

Three different endogenous genes (i.e. actin, lamin A and lamin B2) were knocked down.

Nonspecific effects after siRNA delivery were not observed in cells.

Microinjection of siRNAs into zebrafish cells indicated the capabilty of danio to perform efficient RNAi 


\section{DISCUSSION}

\section{V.1 RNAi against FACE1}

\section{V.1.1 FACE1 was successfully silenced}

In the first part of the thesis I have successfully silenced the human endoprotease FACE1 by RNA interference using the siRNA technique (Elbashir et al., 2001; Harborth et al., 2001). To document silencing after transfection of target specific siRNA a novel technique was introduced. The branched DNA assay (Wang et al., 1997) allowed quantitative determination of the absolute mRNA levels without the pitfalls of RNA extraction, purification and amplification steps as required in real time PCR approaches. Quantification of mRNA amounts was much more sensitive than data obtained by quantitative western blotting. Thus FACE1 and lamin A knockdown could be confirmed on the quantitative mRNA level as well as on the protein level using indirect immunofluorescence microscopy and western blotting.

\section{V.1.2 Accumulation of prelamin A}

In control HeLa cells only small amounts of prelamin A are present. Prelamin A is located to spots within the nuclei, but not to the nuclear lamina (Fig. 4A). Similar results have been reported for control fibroblasts (Goldman et al., 2004). Western blotting of control cells with prelamin A specific antibodies shows no band at the prelamin A position in control cells (Fig. 4B). In contrast in HeLa and MCF7 cells RNAi of FACE1 results in accumulation of relatively large amounts of unprocessed prelamin A at the nuclear membrane both by immunofluorescence (Fig. 4A) and by immunoblotting (Fig. 4B) within 24-48 hours after transfection. These findings support results on the ablation of the murine gene. Ablation of ZMPSTE24 showed an accumulation of prelamin A due to defective processing of prelamin A (Bergo et al., 
2002; Pendas et al., 2002). They further established that prelamin A is a substrate of ZMPSTE24 and that its accumulation results in Hutchinson Gilford Progeria Syndrome related defects, but they do not show whether FACE1 has other substrates in addition to prelamin A (Bergo et al., 2002; Pendas et al., 2002). Recently it was shown, that recombinat human FACE1 processes isoprenylated prelamin A in vitro (Sinensky et al., 2004). Our results with the HeLa cell system are therefore of particular interest. They show that the loss of FACE1 leads only to the strong phenotypes when the cells express the lamin A gene. When HeLa cells are subjected to RNAi knockdown of lamin A (Elbashir et al., 2001; Elbashir et al., 2002; Harborth et al., 2001), such cell populations behave normally when FACE1 is subsequently down regulated by RNAi (Fig. 8A). We have confirmed by the branched DNA assay that such cells indeed have a strongly reduced level of FACE1 mRNA (Fig. 8C). In addition HL60, a cell line that does not express the lamin A gene, shows no effects on FACE1 silencing. This observation supports the conclusion that data obtained show that at least in normal HeLa cells prelamin A is an important substrate of FACE1 and that other substrates, if they exist, are not of immediate importance for cellular growth.

\section{V.1.3 FACE1 knockdown phenotypes and parallels to progeria}

A variety of interesting phenotypic changes have been observed in the siRNA transfected HeLa cells in addition to the accumulation of unprocessed lamin A at the nuclear membrane (Figs. 4-8). Two populations of cells were detected after silencing with FACE1 siRNA. The first population shows changes in nuclear morphology. Nuclei lost their shape, developed folds and lobulation and showed progressive fragmentation

(Fig. 25). Presumably these changes are due to differences between how prelamin A and 
lamin A incorporate into the nuclear lamina. The second population is characterized by multipolar spindles and arrest in mitosis (Fig. 6A). The percentage of cells in mitotic arrest reaches a maximum of $31 \%$ at 48 hours (Fig. 6C). These cells become apoptotic with the percentage of cells in apoptosis reaching $25 \%$ at 72 hours (Fig. 6D). The cause of disturbed spindle architecture and chromosome congression in metaphase is unclear, but presumably results from changes in lamina stability and protein/DNA interactions. Farnesylated, 0-methylated prelamin A could have similar membrane anchoring or hydrophobic interaction properties as the B type lamins (i.e. lamin B1 and lamin B2) and therefore, like B lamins, might remain connected to structures of the nuclear envelope when cells enter mitosis. In contrast, mature lamin A is phosphorylated at the beginning of mitosis, depolymerizes and is soluble in the cytoplasm until it is reassembled on the inner face of the nuclear envelopes in the daughter cells (for review see Goldman et al. (2002) and Moir et al. (2000)).

Fibroblasts from both ZMPSTE24 deficient mice (Pendas et al., 2002) and from HGPS patients (Eriksson et al., 2003) showed abnormalities in nuclear morphology and in this respect are therefore similar to the FACE1 silenced HeLa cells studied here. Fibroblasts from HGPS patients show the same progression of changes in nuclear morphologies, which we observed on FACE1 silencing, starting with folds and herniations, fragmentation of nuclei and ending with micronuleated cells. In addition a high percentage of cells are apoptotic at late passages of HGPS derived fibroblasts (Bridger and Kill, 2004). The most common HGPS mutation was originally identified as an ablation of the FACE1 cleavage site in prelamin A (Eriksson et al., 2003). Accumulation of prelamin $\mathrm{A}$ at the nuclear membrane has been noted in cells from a HGPS patient with this mutation (Goldman et al., 2004), thus paralleling our results with FACE1 silenced HeLa cells. 
The rapid effects on mitosis of FACE1 negative silenced cells indicate a fast intracellular turnover of the protease, since already 24 hours after siRNA delivery a high percentage of cells displayed silencing specific defects and had begun to accumulate prelamin A (Figs. 4A, 7). The results from the CellScreen measurements (Fig. 6E) show that there is a dramatic arrest in cell growth in the FACE1 siRNA treated cells.

Relatively little is known about consequences of mutations in the FACE1 gene, which maps to chromosome 1p34. Agarwal et al. described a Belgian family with mutations in FACE1 (Agarwal et al., 2003). A frameshift mutant introducing an early termination codon provides an inactive enzyme. A missense mutation in a highly conserved region (W340R) yields reduced enzymatic activity. Individuals who were heterozygous for one of these two mutations seem normal, while a patient with both mutations had mandibuloacral dysplasia with progeroid appearance and generalized lipodystrophy and died at age 24. In a very recent study Navarro et al. (2004) sequenced the lamin $\mathrm{A}$ and FACE1 genes from nine patients with restrictive dermopathy (RD). Two had a heterozygous splicing mutation in the lamin A gene, leading to the complete or partial loss of exon 11 in the mRNAs that resulted in a truncated prelamin A. In the other seven patients a unique heterozygous one base pair insertion in exon 9 led to the creation of a premature termination codon in FACE1. In cells grown in culture from patients with FACE1 mutations, loss of normal lamin A expression was observed and in the first three cell divisions up to $50 \%$ of cells had nuclei that were abnormal in shape or size. Navarro et al. (2004) suggest that the FACE1 mutation identified in their study is a necessary but not sufficient genetic defect to cause restrictive dermopathy, since in four families the same mutation was present in one of the parents who was disease free. They further suggest that a second gene may be mutated in patients with restrictive dermopathy. 


\section{V.1.4 Conclusions of FACE1 knockdown experiments}

In conclusion in HeLa cells expressing lamin A, the knockdown of the endoprotease FACE1 has deleterious effects. Since these do not occur after lamin A expression is supressed by $\mathrm{RNAi}$, we propose that the large increase in unprocessed prelamin A may be the direct cause for the lethal phenotype. Although we do not yet understand the cell biological effects in detail, the presence of the permanently farnesylated/0-methylated C-terminal cysteine could have severe effects on lamin A assembly, organisation and function that appear related to those postulated in HGPS patients (Goldman et al., 2004). The HeLa system established here should be very useful for further detailed studies.

\section{V.2 Astrin and mitosis related proteins}

The phenotype seen with astrin siRNA resembled that seen after RNAi of the kinetochore associated protein CENP-E (Harborth et al., 2001). Ablation of this protein by antisense RNA revealed flattening of spindles and fragmentation of spindle poles indicating that CENP-E contributes to the geometry and stability of bipolar spindles (Yao et al., 2000). The absence of any motor domains or ATP binding sites indicates that astrin is not a typical microtubule associated motor protein such as the kinesin motor Eg5 or others, which show the same localisation.

Astrin shows numerous putative phosphorylation sites indicating that astrin is subjected to regulating processes that are different from those of CENP-E. Chang and colleagues (2001) have shown that hMAP126, a protein identical to astrin, is phosphorylated by the $\mathrm{P} 34^{\mathrm{cdc} 2}$-protein kinase, one of the most important kinases in mitosis regulation. This cyclin dependent kinase is essential for controlling correct 
progression of cells through the cell cycle and is activated at the entry to S-phase (Marraccino et al., 1997). This kinase has a regulatory function in mitosis by phosphorylating diverse proteins such as the kinesin related motor protein and others participating in mitotic processes. $\mathrm{P} 34^{\mathrm{cdc} 2}$ phosphorylation of Eg5 regulates its association and is therefore essential for formation of the bipolar spindle apparatus (Blangy et al., 1995). Sustained activity of the P34 ${ }^{\text {cdc2 }}$-protein kinase has been shown to be required for noscapine-induced apoptosis (Ye et al., 2001). This indicates an important role of the $\mathrm{P} 34^{\mathrm{cdc} 2}$-protein kinase in apoptosis induction. In addition the P34 ${ }^{\text {cdc2 }}$-protein kinase phosphorylates hMAP126, an astrin ortholog, in vitro (Chang et al., 2001). The phosphorylation of astrin by a kinase that is known to have essential regulatory functions in mitosis regulation and also apoptosis induction correlates with results in this work. Astrin silencing causes a mitotic arrest followed by apoptosis (compare IIII.2) supporting the view that astrin is an essential part of the mitotic machinery. Silencing of astrin causes a sharp phenotype with targeted cells showing mitotic arrest with multipolar and highly disordered spindle microtubules. After astrin knockdown DNA is replicated and highly increased numbers of tetraploid cells are detected in FACS analysis. However, these chromosomes do not congress to the spindle equator. A similar phenotype has been described for loss of CENP-E function after siRNA mediated gene silencing and antibody microinjection (Harborth et al., 2001, McEwen et al., 2001). Depletion of CENP-E also leads to mitotic arrest of cells that show disordered multipolar spindles. The similar effects on spindle microtubules and chromosome congression (compare McEwen et al., 2001) suggests that both proteins function in the same stage of mitosis. CENP-E is basically involved in ensuring the stability of kinetochore attachment and establishing tension across the centromeres of sister chromatids. CENP-E is also involved in releasing regulatory 
mitotic checkpoint proteins from kinetochores. For example MAD2, a mitotic checkpoint regulation protein, is recruited to kinetochores by low tension (Waters et al., 1998 and 1999) and released after higher tension is established by CENP-E (McEwen et al., 2001 and references therein). Astrin might be involved in these processes since function depletion phenotypes are obviously very similar. In contrast to CENP-E astrin lacks any motor domains, but tends to form organised oligomers (Schnabel, 1998) indicating a function in structural organisation of the spindle apparatus. The high number of PEST motifs is typical for proteins that undergo rapid degradation, suggests a cell cycle dependent expression of astrin combined with a high degree of regulation by mitosis related protein kinases. Thus, a role of astrin as a structural organizing protein establishing bipolar spindle formation or as a checkpoint regulation protein seems likely.

Immunofluorescence analysis of endogenous and GFP-tagged astrin in HeLa cells confirmed the results by Mack and Compton and Chang et al. (Chang et al., 2001; Mack and Compton, 2001, Gruber et al., 2002a,b)). All theses studies a punctate staining in the cytoplasm of interphase cells and colocalization with the spindle apparatus in mitotic cells. In addition astrin was stained in the centromeric region of mitotic cells, indicating a location to kinetochores.

Silencing of the kinetochore related proteins scc1, smc1, smc3, securin and separase indicate the important role of each of the targets for progression through mitosis. Silencing of each of the proteins resulted in growth retardation with cells displaying impaired growth and disturbed chromosome distribution. In each case the bilateral tension at the kinetochores has not been properly established, the kinetochore orientation was aberrant. This leads to severe effects, since correct kinetochore 
function and orientation are essential for mitosis (for review see Hauf and Watanabe, 2004, Earnshaw et al. 2004)

Thus the novel technique of gene silencing using siRNAs allowed the discovery of new aspects concerning the function of the mitosis related proteins astrin and of the other proteins listed in Table 3..

\section{V.3 Anatomy of siRNAs}

\section{V.3.1 Palindromic siRNAs}

Silencing with single stranded RNA was shown with a 5' phosphorylated lamin A/C antisense RNA molecule targeting position 608 (Martinez et al., 2003). More intensive analysis of the RNA revealed its near palindromic sequence features. The self complementarity allows formation of an imperfect RNA duplex with mismatches but with the stability associated with RNA doublestrands. The sequence and structure is given in table lamin1. Active siRNAs and micro RNAs contain 5' phosphates and 3' hydroxyls (Zamore et al. 2000, Boutla et al., 2001, Chiu and Rana 2002).

Therefore the 5'phophorylated, 3'hydroxylated antisense strand of siRNA lamin608 was checked for its ability to form a dsRNA. Subsequently further self complementary regions along the lamin $\mathrm{A} / \mathrm{C}$ region were identified to compare $\mathrm{RNAi}$ effects of appropriate antisense strands. In this study four different palindromic antisense duplexes were tested and their silencing efficiencies were obtained and compared to those of the corresponding s/as RNAs. In three cases the imperfect duplex of the self annealing antisense strand led to a lamin A/C knockdown comparable to classical siRNAs. Only in one case (as/as 94) did the as/as duplex not lead to silencing of lamin $\mathrm{A} / \mathrm{C}$ (see fig. 19 and 21). 


\section{V.3.2 as/as duplexes and dual targeting of lamin $\mathrm{A} / \mathrm{C}$ and emerin}

Heteroduplexes of antisense RNAs targeting lamin A/C were shown to be capable of inducing RNAi. SiRNAs with complementary target region in the lamin A/C sequence were designed and transfected into HeLa SS6 cells. The knockdown efficiencies of as/as duplexes were comparable to those obtained with the individual corresponding s/as siRNAs. Stronger silencing of the as/as duplex when compared to the weaker s/as siRNA indicates the asymmetric assembly of RISC (Schwarz et al., 2003) and its impact on the success of RNAi experiments (see also V.3.3 and figure $\mathrm{X})$. Transfection of single as RNAs also led to a reduction of lamin $\mathrm{A} / \mathrm{C}$, but the efficiency of ssRNA for target specific silencing did not exceed $60 \%$ and was always lower than that obtained with the transfected RNA duplexes.

According to the two target site approach with as/as siRNAs we identified complementary target regions in the sequences of lamin $\mathrm{A} / \mathrm{C}$ and emerin. Examination of silencing efficiencies had to be done by quantitative immunofluorescence microscopy, because quantitative western blot analysis did not yield reliable results. This may be due to dislocation of emerin from the nuclear envelope into the cytoplasm in the absence of lamin A/C (Harbort et al., 2001)). Transfection of the as/as siRNAs against lamin $\mathrm{A} / \mathrm{C}$ and emerin led to a significant and equal reduction of both lamin A/C and of emerin in two cases (as778(L)/as628(E) and as48(L)/as686(E) see Fig. 25). Silencing effects of the individual as RNAs alone can be excluded, since separate transfection of these did not cause any effects on protein expression. The other two pairs of as/as duplexes only reduced one target (lamin A/C, as 1365(L)/as1020(E)) or did not cause (see Figure 25). The results demonstrate that silencing of two genes by use of an appropriate single dsRNA molecule is possible. 


\section{V.3.3 Rescue of siRNAs by sense strand modifications}

For these experiments two inefficient siRNAs were selected, with knockdown levels of less then $20 \%$. These siRNAs contained very stable G/C basepairs in the $5^{\prime}$ ends of their antisense strands. According to the findings of Schwarz et al. (2003) these stable bonds featured a favourable attack of the helicase from the $3^{\prime}$ end of the antisense strand. Thereby the sense strand of the siRNA would be incorporated into RISC and therefore no specific silencing was observed. Schwarz et al. (2003) postulated an asymmetry in the assembly of the RNA/protein complex RISC. Since only one strand of each siRNA is incorporated into RISC (Martinez et al., 2002) an asymmetric assembly means preferred use of a particular strand of the siRNA and not an equal distribution of either sense or antisense strand. (For a proposed mechanism of the asymmetric assembly see the scheme of siRNA and micro RNA processing pathway as defined by Schwarz et al. (Figure 37). SiRNAs and micro RNAs are supposed to be functionally interchangeable and the choice of RNAi or translational repression depends on the degree of target complementarity (Hutvagner and Zamore, 2002, Doench et al., 2003) The disproportional influence of the $5^{\prime}$ nucleotide of the antisense siRNA on silencing efficiencies was noted already in 2003 (Chiu and Rana, 2003) when it was observed that sequence variations in particular in the $5^{\prime}$ region of the siRNA antisense strands had a major impact on silencing functionality. The experimental in vivo proof was performed in parallel to the in vitro approaches described by Schwarz et al., (2003). The tow inefficient siRNAs were modified at the $3^{\prime}$ end of the sense strand to insert $\mathrm{G} / \mathrm{U}$ wobbles or mismatches with the antisense strand. The antisense strand remained unchanged to maintain the target specificity and 
the former low efficiency siRNAs were now successfully used for gene knockdown in cultured cells (Khvorova et al. 2003).

The results presented in this work were consistent with the observations described above. Modification of the siRNAs dramatically increased the efficiencies of both siRNAs, so that knockdown levels of $>75 \%$ could be achieved.

This result underlines the importance of siRNA design in preparation of silencing experiments. The demonstration of how important in particular the $5^{\prime}$-end sequence of the antisense strand of an siRNA is for successful target knockdown should help in the selection of functional siRNA sequences. Another unwanted phenomenon, i.e. the off target activity of the RISC associated sense strand of an siRNA which is occasionally observed (Jackson et al., 2003) may also be excluded by the asymmetric assembly of RISC only with the antisense strand. 


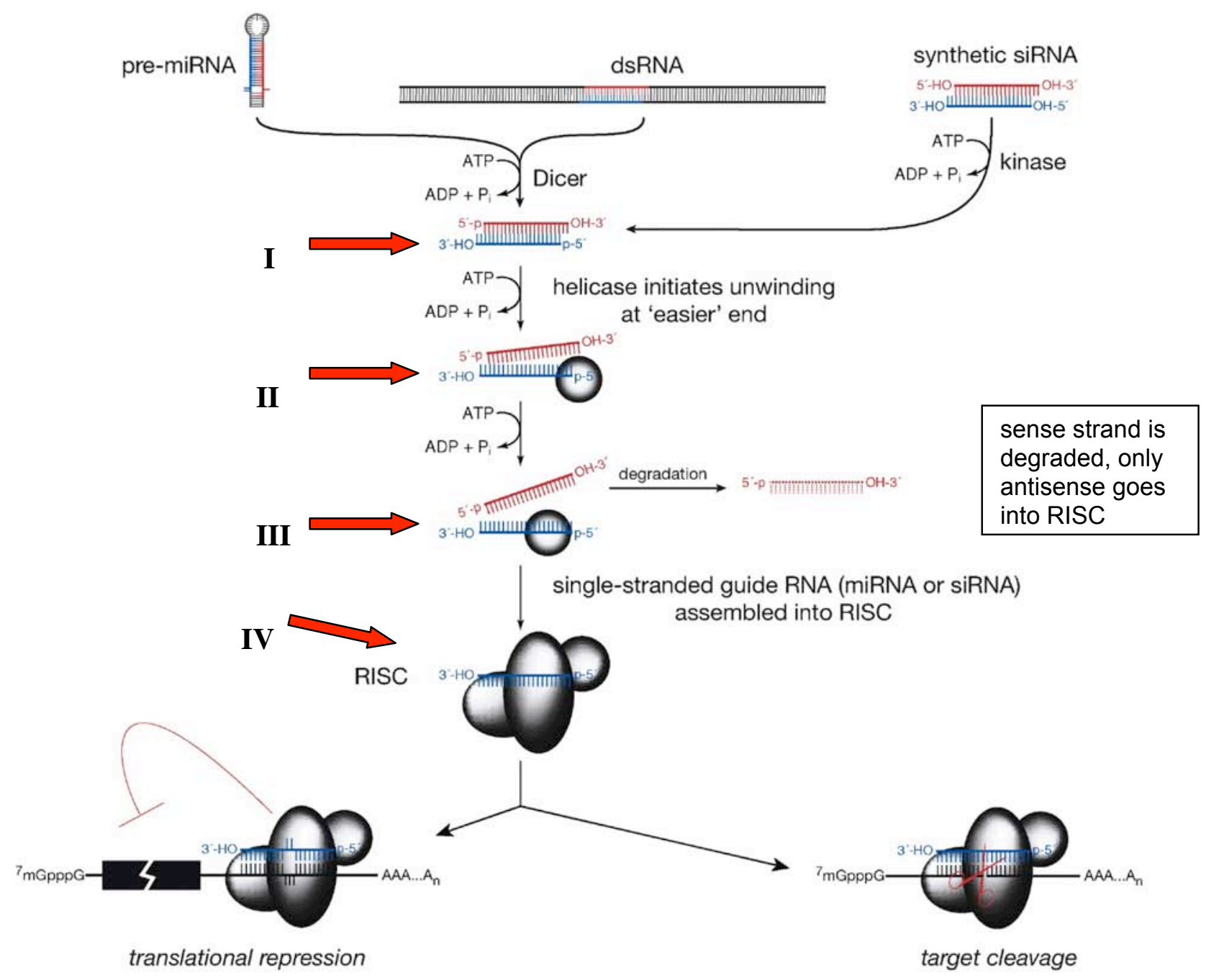

Figure 37: Scheme of micro RNA and RNAi pathways and the asymmetric assembly of RISC. Long dsRNAs, as well as micro RNA precursors, are initially processed by the endonuclease dicer to form $21 \mathrm{nt}$ dsRNAs with 2 nt 3 'overhangs. These dsRNAs have exactly the same structure as synthetic siRNAs (red arrow I). The unwinding of the siRNA by helicase activity (red arrow II) predetermines which strand will be incorporated into RISC (here the desirable antisense strand). The sense strand is removed and subsequently degraded (arrow III) before RISC finally assembles around the antisense strand (arrow IV) tor perform translational repression (micro RNA pathway) or specific mRNA degradation (RNAi pathway).

(modified from Schwarz et al., 2003)

In my Master's thesis (Gruber, 2002) the varying efficiencies of different siRNAs targeting astrin were discussed. The different knockdown levels that could be achieved with three different siRNAs are summarized in table 6. In the original study the secondary structure of the target mRNA was thought to be responsible for RNAi 
functionality since secondary structure predictions indicated intense basepairing in particular in the target regions of less efficient siRNAs.

Table 6: The efficiency of siRNAs targeting human astrin may depend on the $5^{\prime}$ end stability of the antisense strand.

$$
\text { siRNA sequences for astrin knockdown }
$$

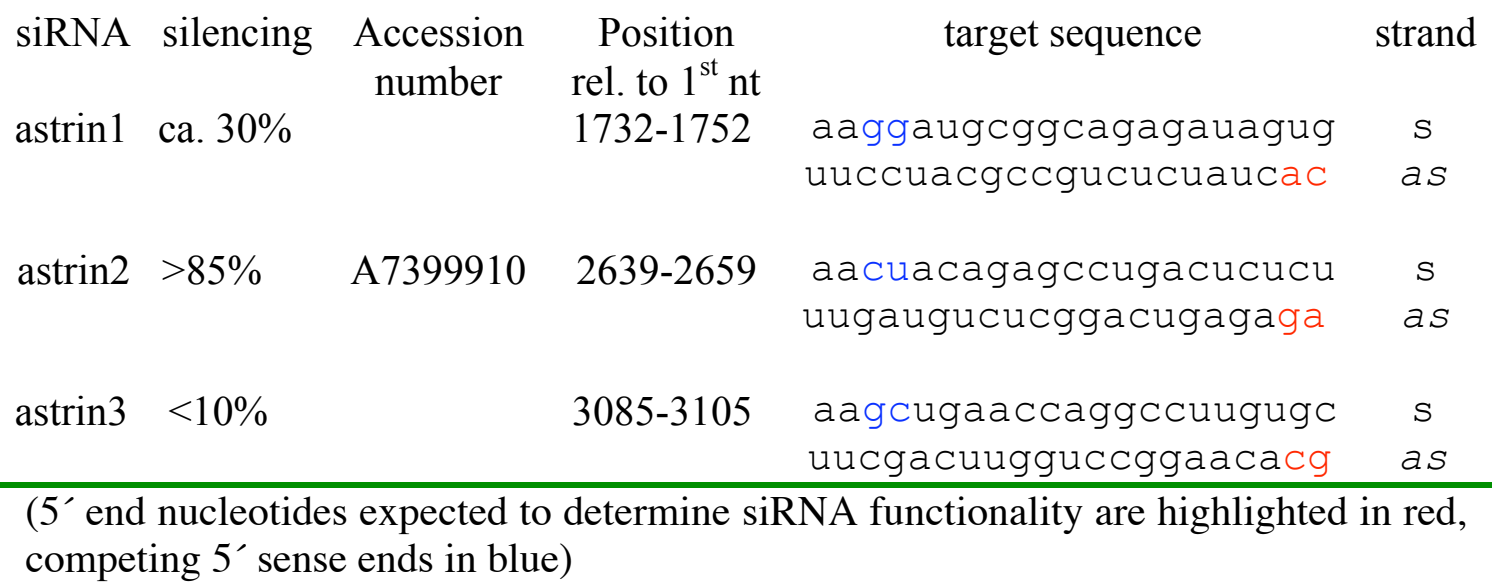

If one takes a closer look at the siRNA sequences in line with the results on siRNA modificatons just discussed, it becomes clear, that the astrin siRNAs obey the rule of weak $5^{\prime}$ antisense ends. In the semi efficient siRNA astrin 1 comparable stabilities of $3^{\prime}$ and $5^{\prime}$ ends lead to competing incorporation into RISC, while the nonefficient siRNA astrin 3 is more stable at the $5^{\prime}$ antisense strand. The third siRNA, astrin2, is less stable at the $5^{\prime}$ end and indeed it showed the highest silencing efficiency in cultured cells.

This effect was also found by comparative analysis of 180 different siRNAs targteing firefly luciferase or human cyclophilin $\mathrm{N}$ in an extensive study of the basic requirements for fuctional RNAi (Reynolds et al., 2003). 


\section{V.4 Pinocytosis mediated siRNA delivery}

It has been shown that siRNAs can be effectively delivered into the cytoplasm of cultured cells by the method of osmotic lysis of pinosomes. The pinocytosis-based method was used to deliver siRNAs to three different cell lines of human and murine origin, i.e. HEK293, HeLa SS6 and SW3T3. In human cells silencing of Eg5, lamin $\mathrm{A} / \mathrm{C}$ and Ki67 target mRNAs was demonstrated. The silencing efficiencies obtained with the pinocytosis method were comparable to those obtained with the conventional liposome-based method. However, in contrast to liposome-mediated siRNA uptake, the pinocytosis-based method avoids technical problems associated with the storage, stability, formulation and reproducibility of liposome reagents.

Our results show that siRNA uptake is time and concentration dependent (Fig. 30), in agreement with an earlier study showing a linear uptake of horseradish peroxidase in fibroblasts over at least 3 hours. Steinman et al. calculate an uptake of $0.0032-0.0035 \%$ of the administered load $/ 10^{6}$ cells/hour (Steinman et al., 1974). In our experiments that would correspond to an uptake of $4 \times 10^{5}$ siRNA molecules/cell/15 minutes during a single round of treatment. Consistent with previous reports, we confirmed that the osmotic shock leading to the lysis of the pinosomes within the cell is required to obtain release of the contents of the pinocytotis vesicles (Okada and Rechsteiner, 1982) (Fig. 28E, F).

Transfection efficiencies can vary remarkably in different cells. In a study using the mammalian expression plasmid pCMV, the most effective vector differed depending on the cell type (Uchida et al., 2002). For siRNA transfection less data is available with emphasis usually placed on transfecting "easy" cell lines such as Hela and K562. For Hela it is clear that high transfection efficiencies can be achieved at lower concentrations of siRNA with the liposome-based method than with the 
pinocytosis method (compare Fig. 29G and Fig. 29E). As for other transfection methods, optimal conditions for uptake of siRNAs by the pinocytosis assay have to be determined for each cell line and it remains to be seen whether cells that are difficult to transfect with liposome-based reagents are more amenable to pinocytosis-based methods. In general extension of the uptake time or use of a double pinocytosis transfection procedure increases the silencing efficiency, but may also lead to a decreased viability. Knockdown levels of $\sim 90 \%$ can be achieved and are comparable with those obtained by more traditional transfection methods, such as cationic liposomes (compare Fig. 28C and 28G) or electroporation (Weil et al., 2002). Calcium phosphate precipitation in the few cases where it has been used seems to result in lower silencing efficiencies with siRNAs (e.g. Weil et al., 2002, Gilmore et al., 2004)).

In summary, pinocytosis mediated transfection of cultured mammalian cells represents a convenient method for the delivery of small RNA molecules important for functional characterisation of gene function.

\section{V.5. RNAi in Danio rerio}

Cultured zebrafish cells of adult and embryonic origin were used in this part of the thesis to test wether RNAi is possible in this system. Use of cell lines allowed biochemical examination of RNAi in adult fish, which is important since previous experiments indicated unspecific effects in zebrafish embryos after injection of dsRNAs into the yolk. The results clearly indicate a difference between the situations the two systems. Expression of exogenous GFP in cells has been successfully inhibited by the corresponding siRNAs while only to unspecific defects were observed when exogenous GFP and corresponding siRNAs were delivered to 
embryos. The malformations seen in embryos were very similar to those reported by other labs after application of ds RNAs which were against exogenous genes or endogenous genes (Zhao et al., 2001, Oates et al., 2000). One publication reports functional RNAi in zebrafish (Dodd et al., 2004) and shows a phenotype very similar to the unspecific effects we observed. This may indicate a general incompatibilty of RNAi in zebrafish embryos, which is currently not undersatnd.

All fish cell lines we used were hard to transfect and transfection efficiencies higher than $30 \%$ were not reached. To confirm functional RNAi it was necessary to apply a reliable siRNA delivery method. By microinjection of siRNAs against different endogenous targets and exogenous GFP it was shown that adult zebrafish cells are capable of performing functional RNAi without displaying the unspecific effects observed in embryos.

Presence of the RNAi machinery in zebrafish has been reported, and a dicer homologue was found in the zebrafish (Lim et al., 2003) but it does not seem to be important during very early embryonic development. Dicer knockout fish survived for 14 days after fertilization while depletion of the maternal dicer mRNA by morpholinos decreased the livespan of the knockout fish to 11 days (Wienholds et al., 2003).

The results presented in previous studies (Zhao et al., 2001, Oates et al., 2000, Dodd et al., 2004) as well as in this thesis show that RNA interference is possible only in zebrafish cell lines, but not in the embryo. The underlying mechanisms, which lead to unspecific defects in the embryo remain to be clarified. 


\section{V.6 Conclusions}

The relevance of gene silencing by means of RNAi as a research tool was demonstrated for a variety of targets. Silencing of the endoprotease FACE1 yielded new insights into the molecular mechanisms of lamin A processing and thereby may provide a novel tool to study the biochemistry of the Hutchinson Gilford Progeria Syndrome as well as to gain insights into the process of normal aging. Silencing of astrin and mitosis related proteins indicated the function of different kinetochore and microtubule associated proteins during mitosis.

New detection methods for phenotypic effects will help to increase data quality and decrease the time required for examination. Examples from this thesis are the branched DNA assay, which allowed quick and accurate quantification of mRNA levels, or the automated microscope system CellScreen, which is ideally suited for growth rate determinations of cells during RNAi experiments since multiple measurements can be made on cells in a single well. Furthermore a novel technique for the delivery of siRNA was established. Transfection by osmotic lysis of siRNA containing pinosomes worked successful on three different target cell lines and may be useful for other cell lines that are hard to transfect by the previously established methods.

Other factors in addition to the target orientation are important when dealing with RNAi. The design of functional siRNAs and the mechanism behind the interference machinery require a much more dtudies before they are finally understood. Although more and more knowledge is gained there are still black boxes to be filled. In this thesis it was shown, that silencing of two targets with a single 
siRNA is possible. Also the asymmetric assembly of RISC was confirmed by rescuing siRNAs against endogenous genes.

Finally it was shown that the zebrafish can do RNAi, at least in cultured cells. The unspecific effects of dsRNAs observed in other studies seem to be restricted to the developing embryo. 


\section{Acknowledgements}

I would like to thank Prof. Mary Osborn for supervising my $\mathrm{PhD}$ thesis, for her continuous support, for the scientific and non-scientific discussions and her patience with the "grasshopper". The same special thanks are addressed to Prof. Klaus Weber, who gave me the opportunity to work in his department.

Thanks also to Prof. Ralf Ficner for being the second referee of this thesis and to Prof. Tom Tuschl for supervision, support and fruitful discussions.

I would also like to thank Dr. Jens Harborth and Dr. Sayda Elbashir for all their help in the beginning and the great introduction into the exciting field of RNAi.

Special thanks are addressed to Markus Hossbach and Heiko Manninga for great collaboration on the lamin and zebrafish projects, lots of discussions and their company during all the ups and downs.

I thank Tina Lampe and Kerstin Bartscherer for collaboration and discussions, Guido Böse for help with the pinocytosis project, Heinz-Jürgen Dehne for a lot of help and funny afternoons in the cell lab.

Thanks also to Dr. Alexander Zimek, Dr. Anton Karabinos, Jürgen Schünemann, Tomma Eisbein and all the other members of our department for a great working atmosphere.

Special thanks are adressed to all my friends and to my family, in particular to my parents, for for help and support and fun.

Last, but not least, I want to address my very special thanks to Eva, mi corazon, for support, for being with me, for her love. 


\section{REFERENCES}

Abrieu, A., Kahana, J. A., Wood, K. W. and Cleveland, D. W. (2000). CENP-E as an essential component of the mitotic checkpoint in vitro. Cell 102, 817-26.

Agarwal, A. K., Fryns, J. P., Auchus, R. J. and Garg, A. (2003). Zinc metalloproteinase, ZMPSTE24, is mutated in mandibuloacral dysplasia. Hum Mol Genet 12, 1995-2001.

Alexandropoulos, K., Cheng, G. and Baltimore, D. (1995). Proline-rich sequences that bind to Src homology 3 domains with individual specificities. Proceedings of the National Academy of Sciences of the U.S.A. 92, 3110-3114.

Auffrey, C. and Rougeon, F. (1980). Purification of mouse immunoglobulin heavychain messenger RNAs from total myeloma tumor RNA. European Journal of Cell Biology 107, 303-314.

Banks, J. D. and Heald, R. (2001). Chromosome movement: dynein-out at the kinetochore. Curr Biol 11, R128-31.

Bass, B.L. (2001) RNA interference, the short answer. Nature, 411, 428-429.

Baum P, Furlong C, Byers B. Yeast gene required for spindle pole body duplication: homology of its product with Ca2+-binding proteins. Proc Natl Acad Sci U S A. 1986 Aug;83(15):5512-6.

Bergo, M. O., Gavino, B., Ross, J., Schmidt, W. K., Hong, C., Kendall, L. V., Mohr, A., Meta, M., Genant, H., Jiang, Y. et al. (2002). Zmpste24 deficiency in mice causes spontaneous bone fractures, muscle weakness, and a prelamin A processing defect. Proc Natl Acad Sci U S A 99, 13049-13054.

Bernstein E, Denli AM, Hannon GJ. The rest is silence. RNA. 2001 Nov;7(11):150921. Review.

Bernstein, E., Caudy, A.A., Hammond, S.M. and Hannon, G.J. (2001) Role for a bidentate ribonuclease in the initiation step of RNA interference. Nature, 409, 363366.

Billy E, Brondani V, Zhang H, Muller U, Filipowicz W. Specific interference with gene expression induced by long, double-stranded RNA in mouse embryonal teratocarcinoma cell lines. Proc Natl Acad Sci U S A. 2001 Dec 4;98(25):14428-33.

Blangy, A., H.A. Lane, P. d'Herin, M. Harper, M. Kress, and E.A. Nigg. 1995. Phosphorylation by p34cdc2 regulates spindle association of human Eg5, a kinesinrelated motor essential for bipolar spindle formation in vivo. Cell 83:1159-1169.

Bonne G, Di Barletta MR, Varnous S, Becane HM, Hammouda EH, Merlini L, Muntoni F, Greenberg CR, Gary F, Urtizberea JA, Duboc D, Fardeau M, Toniolo D, 
Schwartz K. Mutations in the gene encoding lamin A/C cause autosomal dominant Emery-Dreifuss muscular dystrophy. Nat Genet. 1999 Mar;21(3):285-8.

Boonanuntanasarn S, Yoshizaki G, Takeuchi T. Specific gene silencing using small interfering RNAs in fish embryos. Biochem Biophys Res Commun. 2003 Oct 31;310(4):1089-95.

Boutla A, Kalantidis K, Tavernarakis N, Tsagris M, Tabler M. Induction of RNA interference in Caenorhabditis elegans by RNAs derived from plants exhibiting posttranscriptional gene silencing. Nucleic Acids Res. 2002 Apr 1;30(7):1688-94.

Bradford, M. M. (1976). A rapid and sensitive method for the quantitation of microgramm quantities of protein utilizing the principle of protein-dye binding. Anal. Biochem. 72, 248-254.

Bridger, J. M. and Kill, I. R. (2004). Aging of Hutchinson-Gilford progeria syndrome fibroblasts is characterised by hyperproliferation and increased apoptosis. Exp Gerontol 39, 717-724.

Broers, J. L., Machiels, B. M., Kuijpers, H. J., Smedts, F., van den Kieboom, R., Raymond, Y. and Ramaekers, F. C. (1997). A- and B-type lamins are differentially expressed in normal human tissues. Histochem Cell Biol 107, 505-517.

Brummelkamp TR, Bernards R, Agami R. A System for Stable Expression of Short Interfering RNAs in Mammalian Cells.

Brummelkamp, T.R., R. Bernards, and R. Agami. 2002. A system for stable expression of short interfering RNAs in mammalian cells. Science 296:550-553.

Burke, B. and Stewart, C. L. (2002). Life at the edge: the nuclear envelope and human disease. Nat Rev Mol Cell Biol 3, 575-585.

Cao, H. and Hegele, R. A. (2003). LMNA is mutated in Hutchinson-Gilford progeria (MIM 176670) but not in Wiedemann-Rautenstrauch progeroid syndrome (MIM 264090). J Hum Genet 48, 271-274.

Caplen NJ, Parrish S, Imani F, Fire A, Morgan RA. Specific inhibition of gene expression by small double-stranded RNAs in invertebrate and vertebrate systems. Proc Natl Acad Sci U S A. 2001 Aug 14;98(17):9742-7.

Carthew RW. Gene silencing by double-stranded RNA. Curr Opin Cell Biol. 2001 Apr;13(2):244-8. Review

Celis JE, Cell Biology, a laboratory handbook (1998), Academic Press, $2^{\text {nd }}$ Ed., Volume 1,:230-231

Cerutti L, Mian N, Bateman A. Domains in gene silencing and cell differentiation proteins: the novel PAZ domain and redefinition of the Piwi domain. Trends Biochem Sci. 2000 Oct;25(10):481-2. 
Chang, M. S., Huang, C. J., Chen, M. L., Chen, S. T., Fan, C. C., Chu, J. M., Lin, W. C. and Yang, Y. C. (2001). Cloning and characterization of hMAP126, a new member of mitotic spindle-associated proteins. Biochem Biophys Res Commun 287, 116-21.

Chen L, Lee L, Kudlow BA, Dos Santos HG, Sletvold O, Shafeghati Y, Botha EG, Garg A, Hanson NB, Martin GM, Mian IS, Kennedy BK, Oshima J. LMNA mutations in atypical Werner's syndrome. Lancet. 2003 Aug 9;362(9382):440-5.

Chen, L., Lee, L., Kudlow, B. A., Dos Santos, H. G., Sletvold, O., Shafeghati, Y., Botha, E. G., Garg, A., Hanson, N. B., Martin, G. M. et al. (2003). LMNA mutations in atypical Werner's syndrome. Lancet 362, 440-445.

Chiu YL, Rana TM. siRNA function in RNAi: a chemical modification analysis. RNA. 2003 Sep;9(9):1034-48.

Clemens MJ, Elia A. The double-stranded RNA-dependent protein kinase PKR: structure and function. J Interferon Cytokine Res. 1997b Sep;17(9):503-24. Review

Clemens MJ. PKR--a protein kinase regulated by double-stranded RNA. Int J Biochem Cell Biol. 1997a Jul;29(7):945-9. Review

Cleveland DW, Fischer SG, Kirschner MW, Laemmli UK. Peptide mapping by limited proteolysis in sodium dodecyl sulfate and analysis by gel electrophoresis.

Cogoni, C. and Macino, G. (2000) Post-transcriptional gene silencing across kingdoms. Curr. Opin. Genet. Dev., 10, 638-643.

Collet B, Secombes CJ. Type I-interferon signalling in fish. Fish Shellfish Immunol. 2002 May;12(5):389-97. Review.

Compton DA. Spindle assembly in animal cells. Annu Rev Biochem. 2000;69:95114. Review

Crick, F.H.C. (1953). The rourier transform of a coiled-coil. Acta. Cryst. 6: 685-697

Czauderna F, Fechtner M, Dames S, Aygun H, Klippel A, Pronk GJ, Giese K, Kaufmann J. Structural variations and stabilising modifications of synthetic siRNAs in mammalian cells. Nucleic Acids Res. 2003 Jun 1;31(11):2705-16.

De Sandre-Giovannoli, A., Bernard, R., Cau, P., Navarro, C., Amiel, J., Boccaccio, I., Lyonnet, S., Stewart, C. L., Munnich, A., Le Merrer, M. et al. (2003). Lamin a truncation in Hutchinson-Gilford progeria. Science 300, 2055.

Desai, A., Murray, A., Mitchison, T.J. and Walczak, C.E. (1999) The use of Xenopus egg extracts to study mitotic spindle assembly and function in vitro. Methods Cell Biol., 61, 385-412.

Dodd A, Chambers SP, Love DR. Short interfering RNA-mediated gene targeting in the zebrafish. FEBS Lett. 2004 Mar 12;561(1-3):89-93. 
Dorsett YTuschl T.siRNAs: applications in functional genomics and potential as therapeutics. Nat Rev Drug Discov. 2004 Apr;3(4):318-29. Review.

Elbashir SM, Martinez J, Patkaniowska A, Lendeckel W, Tuschl T. Functional anatomy of siRNAs for mediating efficient RNAi in Drosophila melanogaster embryo lysate. EMBO J. 2001 Dec 3;20(23):6877-88.

Elbashir, S. M., Harborth, J., Lendeckel, W., Yalcin, A., Weber, K. and Tuschl, T. (2001). Duplexes of 21-nucleotide RNAs mediate RNA interference in cultured mammalian cells. Nature 411, 494-498.

Elbashir, S. M., Harborth, J., Weber, K. and Tuschl, T. (2002). Analysis of gene function in somatic mammalian cells using small interfering RNAs. Methods 26, 199213.

Elbashir, S.M., Lendeckel, W. and Tuschl, T. (2001) RNA interference is mediated by 21-and 22-nucleotide RNAs. Genes Dev., 15, 188-200.

Emery AE, Dreifuss FE. Unusual type of benign x-linked muscular dystrophy. J Neurol Neurosurg Psychiatry. 1966 Aug;29(4):338-42.

Emery AE. Emery-Dreifuss muscular dystrophy - a 40 year retrospective. Neuromuscul Disord. 2000 Jun;10(4-5):228-32. Review.

Eriksson, M., Brown, W. T., Gordon, L. B., Glynn, M. W., Singer, J., Scott, L., Erdos, M. R., Robbins, C. M., Moses, T. Y., Berglund, P. et al. (2003). Recurrent de novo point mutations in lamin A cause Hutchinson-Gilford progeria syndrome. Nature 423, 293-298.

Felgner PL, Ringold GM Cationic liposome-mediated transfection. Nature. 1989 Jan 26;337(6205):387-8.

Fire A, Xu S, Montgomery MK, Kostas SA, Driver SE, Mello CC. Potent and specific genetic interference by double-stranded RNA in Caenorhabditis elegans. Nature. 1998 Feb 19;391(6669):806-11.

Fire, A. (1999) RNA-triggered gene silencing. Trends Genet., 15, 358-363.

Flemming, W. (1879) Beiträge zur Kenntnis der Zelle und ihrer Lebenserscheinungen. Arch. Mikroskop. Anat. 18: 151-259

Fraser, A.G., Kamath, R.S., Zipperlen, P., Martinez-Campos, M., Sohrmann, M. and Ahringer, J. (2000) Functional genomic analysis of C-elegans chromosome I by systematic RNA interference. Nature, 408, 325-330.

Fuchs E, Weber K. Intermediate filaments: structure, dynamics, function, and disease. Annu Rev Biochem. 1994;63:345-82. Review. 
Garg A, Speckman RA, Bowcock AM. Multisystem dystrophy syndrome due to novel missense mutations in the amino-terminal head and alpha-helical rod domains of the lamin A/C gene. Am J Med. 2002 May;112(7):549-55.

Garrus JE, von Schwedler UK, Pornillos OW, Morham SG, Zavitz KH, Wang HE, Wettstein DA, Stray KM, Cote M, Rich RL, Myszka DG, Sundquist WI. Tsg101 and the vacuolar protein sorting pathway are essential for HIV-1 budding. Cell. 2001 Oct 5;107(1):55-65.

Geiss G, Jin G, Guo J, Bumgarner R, Katze MG, Sen GC. A comprehensive view of regulation of gene expression by double-stranded RNA-mediated cell signaling. J Biol Chem. 2001 Aug 10;276(32):30178-82

Georgiou G, Valax P. Isolating inclusion bodies from bacteria. Methods Enzymol. 1999;309:48-58

Gerace, L. and Blobel, G. (1980). The nuclear envelope lamina is reversibly depolymerized during mitosis. Cell 19, 277-287.

Gilmore IR, Fox SP, Hollins AJ, Sohail M, Akhtar S. The design and exogenous delivery of siRNA for post-transcriptional gene silencing. J Drug Target. 2004 Jul;12(6):315-40.

Glotzer M. Mitosis: don't get mad, get even. Curr Biol. 1996 Dec 1;6(12):1592-4. Review.

Goldman, R. D., Gruenbaum, Y., Moir, R. D., Shumaker, D. K. and Spann, T. P. (2002). Nuclear lamins: building blocks of nuclear architecture. Genes Dev 16, 533 547.

Goldman, R. D., Shumaker, D. K., Erdos, M. R., Eriksson, M., Goldman, A. E., Gordon, L. B., Gruenbaum, Y., Khuon, S., Mendez, M., Varga, R. et al. (2004). Accumulation of mutant lamin A causes progressive changes in nuclear architecture in Hutchinson-Gilford progeria syndrome. Proc Natl Acad Sci U S A 101, 8963-8968.

Gonczy, P., Echeverri, C., Oegema, K., Coulson, A., Jones, S.J.M., Copley, R.R., Duperon, J., Oegema, J., Brehm, M., Cassin, E., Hannak, E., Kirkham, M., Pichler, S., Flohrs, K., Goessen, A., Leidel, S., Alleaume, A.M., Martin, C., Ozlu, N., Bork, P. and Hyman, A.A. (2000) Functional genomic analysis of cell division in C-elegans using RNAi of genes on chromosome III. Nature, 408, 331-336.

Grassman J, Haas R. Development of an immunoassay to detect hemoglobin adducts formed by benzene exposure.

Gruber, Jens; Functional gene analysis in cultured mammalian cells using siRNA mediated gene silencing shows that the human coiled-coil protein astrin is essential for mitosis and cell survival (International Max-Planck Research School Molecular Biology, 2002, Master's Thesis) 
Gruber J, Harborth J, Schnabel J, Weber K, Hatzfeld M. (2002) The mitotic-spindleassociated protein astrin is essential for progression through mitosis. J Cell Sci. Nov 1;115(Pt 21):4053-9.

Gruber J, Boese G, Tuschl T, Osborn M, Weber K. RNA interference by osmotic lysis of pinosomes: liposome-independent transfection of siRnas into mammalian cells. Biotechniques. 2004 Jul;37(1):96-102.

Gruber J, Lampe T, Osborn M and Weber K RNAi of FACE1 protease results in growth inhibition of human cells expressing lamin A; implications for HutchinsonGilford Progeria Syndrome J Cell Science (in press)

Gueth-Hallonet, C., Osborn, M. and Compton, D.A. (1999) NuMA. In Kreis, T. and Vale, R. (ed.), Guidebook to the cytoskeletal and motor proteins. Oxford Univ. Press, Oxford, pp. 265-268.

Hamilton, A.J. \& Baulcombe, D.C. A species of small antisense RNA in posttranscriptional gene silencing in plants. Science 286, 950-952 (1999).

Hammond, S.M., Bernstein, E., Beach, D. \& Hannon, G.J. An RNA-directed nuclease mediates post-transcriptional gene silencing in Drosophila cells. Nature 404, 293-296 (2000).

Hammond, S.M., Caudy, A.A. and Hannon, G.J. (2001) Post-transcriptional gene silencing by double-stranded RNA. Nature Reviews Genetics, 2, 110-119.

Hanahan D, Jessee J, Bloom FR. Plasmid transformation of Escherichia coli and other bacteria. Methods Enzymol. 1991;204:63-113. Review

Harborth J, Elbashir SM, Vandenburgh K, Manninga H, Scaringe SA, Weber K, Tuschl T. Sequence, chemical, and structural variation of small interfering RNAs and short hairpin RNAs and the effect on mammalian gene silencing. Antisense Nucleic Acid Drug Dev. 2003 Apr;13(2):83-105.

Harborth, J., Elbashir, S. M., Bechert, K., Tuschl, T. and Weber, K. (2001). Identification of essential genes in cultured mammalian cells using small interfering RNAs. J Cell Sci 114, 4557-65.

Heald, R., Tournebize, R., Blank, T., Sandaltzopoulos, R., Becker, P., Hyman, A. and Karsenti, E. (1996). Self-organization of microtubules into bipolar spindles around artificial chromosomes in Xenopus egg extracts. Nature 382, 420-5.

Hennekes, H. and Nigg, E. A. (1994). The role of isoprenylation in membrane attachment of nuclear lamins. A single point mutation prevents proteolytic cleavage of the lamin A precursor and confers membrane binding properties. J Cell Sci 107, 10191029.

Hirokawa, N., Noda, Y. and Okada, Y. (1998a). Kinesin and dynein superfamily proteins in organelle transport and cell division. Curr Opin Cell Biol 10, 60-73. 
Hirokawa, N., Noda, Y. and Okada, Y. (1998b). Kinesin and dynein superfamily proteins in organelle transport and cell division. Current Opinion in Cell Biology 10, 60-73.

Hirose, K. and Amos, L. A. (1999). Three-dimensional structure of motor molecules. Cell Mol Life Sci 56, 184-99.

Howell, B. J., McEwen, B. F., Canman, J. C., Hoffman, D. B., Farrar, E. M., Rieder, C. L. and Salmon, E. D. (2001). Cytoplasmic dynein/dynactin drives kinetochore protein transport to the spindle poles and has a role in mitotic spindle checkpoint inactivation. J Cell Biol 155, 1159-72.

Hoyt MA, Geiser JR. Genetic analysis of the mitotic spindle. Annu Rev Genet. 1996;30:7-33. Review

Hsieh DJ, Liao CF. Zebrafish M2 muscarinic acetylcholine receptor: cloning, pharmacological characterization, expression patterns and roles in embryonic bradycardia. Br J Pharmacol. 2002 Nov;137(6):782-92.

Hutvagner G, McLachlan J, Pasquinelli AE, Balint E, Tuschl T, Zamore PD. A cellular function for the RNA-interference enzyme Dicer in the maturation of the let-7 small temporal RNA. Science. 2001 Aug 3;293(5531):834-8.

Jensen I, Larsen R, Robertsen B. An antiviral state induced in Chinook salmon embryo cells (CHSE-214) by transfection with the double-stranded RNA poly I:C. Fish Shellfish Immunol. 2002 Nov;13(5):367-78.

Joshi, H. C. (1998). Microtubule dynamics in living cells. Current Opinion in Cell Biology 10, 35-44.

Karsenti, E. and Vernos, I. (2001). The mitotic spindle: a self-made machine. Science 294, 543-7.

Kennerdell JR, Carthew RW. Use of dsRNA-mediated genetic interference to demonstrate that frizzled and frizzled 2 act in the wingless pathway. Cell. 1998 Dec 23;95(7):1017-26.

Ketting RF, Haverkamp TH, van Luenen HG, Plasterk RH. Mut-7 of C. elegans, required for transposon silencing and RNA interference, is a homolog of Werner syndrome helicase and RNaseD. Cell. 1999 Oct 15;99(2):133-41.

Key B, Devine ZCAArafish as an experimental model: strategies for developmental and molecular neurobiology studies. Methods Cell Sci. 2003;25(12):1-6. Review.

Khvorova A, Reynolds A, Jayasena SD. Functional siRNAs and miRNAs exhibit strand bias. Cell. 2003 Oct 17;115(2):209-16. Erratum in: Cell. 2003 Nov 14;115(4):505. 
Kim, A. J. and Endow, S. A. (2000). A kinesin family tree. J Cell Sci 113 Pt 21, 36812.

Kim, S.K. (2001) Functional genomics: The worm scores a knockout. Curr. Biol., 11, R85-7.

Kirchner, J., Woehlke, G. and Schliwa, M. (1999). Universal and unique features of kinesin motors: insights from a comparison of fungal and animal conventional kinesins. Biol Chem 380, 915-21.

Kirschner MW, Mitchison T. Microtubule dynamics. Nature. 1986 Dec 1831;324(6098):621.

Kyhse-Andersen, J. (1984). Electroblotting of multiple gels: a simple apparatus without buffer tank for rapid transfer of proteins from polyacrylamide to nitrocellulose. J Biochem Biophys Methods 10, 203-209.

Leaman DW, Salvekar A, Patel R, Sen GC, Stark GR. Free in PMC A mutant cell line defective in response to double-stranded RNA and in regulating basal expression of interferon-stimulated genes. Proc Natl Acad Sci U S A. 1998 Aug 4;95(16):9442-7

Lim LP, Glasner ME, Yekta S, Burge CB, Bartel DP. Vertebrate microRNA genes. Science. 2003 Mar 7;299(5612):1540.

Liu J, Carmell MA, Rivas FV, Marsden CG, Thomson JM, Song JJ, Hammond SM, Joshua-Tor L, Hannon GJ. Argonaute2 is the catalytic engine of mammalian RNAi. Science. 2004 Sep 3;305(5689):1437-41. Epub 2004 Jul 29.

Lockhart A, Cross RA. Kinetics and motility of the Eg5 microtubule motor.Biochemistry. 1996 Feb 20;35(7):2365-73.

Lombillo VA, Nislow C, Yen TJ, Gelfand VI, McIntosh JR. Antibodies to the kinesin motor domain and CENP-E inhibit microtubule depolymerization-dependent motion of chromosomes in vitro. J Cell Biol. 1995 Jan;128(1-2):107-15

Lombillo, V. A., Nislow, C., Yen, T. J., Gelfand, V. I. and McIntosh, J. R. (1995). Antibodies to the kinesin motor domain and CENP-E inhibit microtubule depolymerization-dependent movement of chromosomes in vitro. Journal of Cell Biology 128, 107-115.

Lu KP, Kemp BE, Means AR. Identification of substrate specificity determinants for the cell cycle-regulated NIMA protein kinase. J Biol Chem. 1994 Mar 4;269(9):66037.

Lupas A. Coiled-coils: new structures and new functions. Trends Biochem Sci. 1996 Oct;21(10):375-82. Review.

Mack, G. J. and Compton, D. A. (2001). Analysis of mitotic microtubule-associated proteins using mass spectrometry identifies astrin, a spindle-associated protein. Proc Natl Acad Sci U S A 98, 14434-9. 
Maeda, I., Kohara, Y., Yamamoto, M. and Sugimoto, A. (2001) Large-scale analysis of gene function in Caenorhabditis elegans by high-throughput RNAi. Curr. Biol., 11, 171-176.

Maney, T., Ginkel, L. M., Hunter, A. W. and Wordeman, L. (2000). The kinetochore of higher eucaryotes: a molecular view. Int Rev Cytol 194, 67-131.

Marraccino RL, Firpo EJ, Roberts JM. Activation of the p34 CDC2 protein kinase at the start of S phase in the human cell cycle. Mol Biol Cell. 1992 Apr;3(4):389-401.

Martinez J, Patkaniowska A, Urlaub H, Luhrmann R, Tuschl T. Single-stranded antisense siRNAs guide target RNA cleavage in RNAi. Cell. 2002 Sep 6;110(5):56374.

Martinez J, Tuschl T. RISC is a 5' phosphomonoester-producing RNA endonuclease. Genes Dev. 2004 May 1;18(9):975-80. Epub 2004 Apr 22.

Martinez J, Tuschl T. RISC is a 5' phosphomonoester-producing RNA endonuclease. Genes Dev. 2004 May 1;18(9):975-80. Epub 2004 Apr 22.

McEwen BF, Chan GK, Zubrowski B, Savoian MS, Sauer MT, Yen TJ. CENP-E is essential for reliable bioriented spindle attachment, but chromosome alignment can be achieved via redundant mechanisms in mammalian cells. Mol Biol Cell. 2001 Sep;12(9):2776-89.

Meluh PB, Rose MD. KAR3, a kinesin-related gene required for yeast nuclear fusion. Cell. 1990 Mar 23;60(6):1029-41.

Merdes A, Ramyar K, Vechio JD, Cleveland DW. A complex of NuMA and cytoplasmic dynein is essential for mitotic spindle assembly. Cell. 1996 Nov $1 ; 87(3): 447-58$

Miki, H., Setou, M., Kaneshiro, K. and Hirokawa, N. (2001). All kinesin superfamily protein, KIF, genes in mouse and human. Proc Natl Acad Sci U S A 98, 7004-11.

Mirzayan C, Copeland CS, Snyder M. The NUF1 gene encodes an essential coiledcoil related protein that is a potential component of the yeast nucleoskeleton. J Cell Biol. 1992 Mar;116(6):1319-32

Moir, R. D., Spann, T. P., Lopez-Soler, R. I., Yoon, M., Goldman, A. E., Khuon, S. and Goldman, R. D. (2000). Review: the dynamics of the nuclear lamins during the cell cycle-- relationship between structure and function. J Struct Biol 129, 324-334.

Montgomery MK, Fire A. Double-stranded RNA as a mediator in sequence-specific genetic silencing and co-suppression. Trends Genet. 1998 Jul;14(7):255-8. Review.

Montgomery MK, Xu S, Fire A. RNA as a target of double-stranded RNA-mediated genetic interference in Caenorhabditis elegans. Proc Natl Acad Sci U S A. 1998 Dec 22;95(26):15502-7. 
Moore, J. D. and Endow, S. A. (1996). Kinesin proteins: a phylum of motors for microtubule-based motility. Bioessays 18, 207-219.

Moritz M, Braunfeld MB, Sedat JW, Alberts B, Agard DA. Microtubule nucleation by gamma-tubulin-containing rings in the centrosome. Nature. 1995 Dec 7;378(6557):638-40.

Mounkes, L., Kozlov, S., Burke, B. and Stewart, C. L. (2003). The laminopathies: nuclear structure meets disease. Curr Opin Genet Dev 13, 223-230.

Muchir A, Bonne G, van der Kooi AJ, van Meegen M, Baas F, Bolhuis PA, de Visser $\mathrm{M}$, Schwartz K. Identification of mutations in the gene encoding lamins $\mathrm{A} / \mathrm{C}$ in autosomal dominant limb girdle muscular dystrophy with atrioventricular conduction disturbances (LGMD1B). Hum Mol Genet. 2000 May 22;9(9):1453-9.

Mullis KB, Faloona FA.Specific synthesis of DNA in vitro via a polymerasecatalyzed chain reaction. Methods Enzymol. 1987;155:335-50.

Navarro, C. L., De Sandre-Giovannoli, A., Bernard, R., Boccaccio, I., Boyer, A., Genevieve, D., Hadj-Rabia, S., Gaudy-Marqueste, C., Smitt, H. S., Vabres, P. et al. (2004). Lamin A and ZMPSTE24 (FACE-1) defects cause nuclear disorganization and identify restrictive dermopathy as a lethal neonatal laminopathy. Hum Mol Genet 13, 2493-2503.

Nedelec, F. J., Surrey, T., Maggs, A. C. and Leibler, S. (1997). Self-organization of microtubules and motors. Nature 389, 305-8.

Nehls, M., Luno, K., Schorpp, M., Pfeifer, D., Krause, S., Matysiak-Scholze, U., Dierbach, H. and Boehm, T. (1995). YAC/P1 contigs defining the location of 56 microsatellite markers and several genes across $3.4 \mathrm{cM}$ interval on mouse chromosome 11. Mammalian Genome 6, 321-331.

Nicklas, R. B., Ward, S. C. and Gorbsky, G. J. (1995). Kinetochore chemistry is sensitive to tension and may link mitotic forces to a cell cycle checkpoint. Journal of Cell Biology 130, 929-939.

Nigg EA, Blangy A, Lane HA Dynamic changes in nuclear architecture during mitosis: on the role of Protein phosphorylation in spindle assembly and chromosome segregation. Exp Cell Res. 1996 Dec 15;229(2):174-80. Review.

Nigg, E.A. (2001) Mitotic kinases as regulators of cell division and its checkpoints. Nat. Rev. Mol. Cell Biol., 2, 21-32.

Nislow C, Lombillo VA, Kuriyama R, McIntosh JR. A plus-end-directed motor enzyme that moves antiparallel microtubules in vitro localizes to the interzone of mitotic spindles. Nature. 1992 Oct 8;359(6395):543-7.

Novelli, G., Muchir, A., Sangiuolo, F., Helbling-Leclerc, A., D'Apice, M. R., Massart, C., Capon, F., Sbraccia, P., Federici, M., Lauro, R. et al. (2002). Mandibuloacral 
dysplasia is caused by a mutation in LMNA-encoding lamin A/C. Am J Hum Genet $71,426-431$.

Oates AC, Bruce AE, Ho RK. Too much interference: injection of double-stranded RNA has nonspecific effects in the zebrafish embryo. Dev Biol. 2000 Aug $1 ; 224(1): 20-8$.

Okada, C.Y., and M. Rechsteiner. 1982. Introduction of macromolecules into cultured mammalian cells by osmotic lysis of pinocytic vesicles. Cell 29:33-41.

Osborn M, Geisler N, Shaw G, Sharp G, Weber K. Intermediate filaments.Cold Spring Harb Symp Quant Biol. 1982;46 Pt 1:413-29

Osborn, M., Debus, E. and Weber, K. (1984). Monoclonal antibodies specific for vimentin. Eur J Cell Biol 34, 137-43.

Park, R.D., P.C. Sullivan, and B. Storrie. 1988. Hypertonic sucrose inhibition of endocytic transport suggests multiple early endocytic compartments. J Cell Physiol. 135:443-450.

Parrish, S., Fleenor, J., Xu, S., Mello, C. \& Fire, A. Functional anatomy of a dsRNA trigger: Differential requirement for the two trigger strands in RNA Interference. Mol. Cell 6, 1077-1087 (2000

Pendas, A. M., Zhou, Z., Cadinanos, J., Freije, J. M., Wang, J., Hultenby, K., Astudillo, A., Wernerson, A., Rodriguez, F., Tryggvason, K. et al. (2002). Defective prelamin A processing and muscular and adipocyte alterations in Zmpste24 metalloproteinase-deficient mice. Nat Genet 31, 94-99.

Peters KL, Smith HL, Stark GR, Sen GC. Free in PMC IRF-3-dependent, NFkappa Band JNK-independent activation of the 561 and IFN-beta genes in response to doublestranded RNA. Proc Natl Acad Sci U S A. 2002 Apr 30;99(9):6322-7.

Pietromonaco SF, Seluja GA, Elias L. Identification of enzymatically active $\mathrm{Ca} 2+$ /calmodulin-dependent protein kinase in centrosomes of hemopoietic cells. Blood Cells Mol Dis. 1995;21(1):34-41.

Porter, A. (1998) Controlling your losses: conditional gene silencing in mammals. Trends Genet., 14, 73-79.

Rechsteiner, M. and Rogers, S. W. (1996). PEST sequences and regulation by proteolysis. Trends in Biochemical Sciences 21, 267-271.

Reynolds A, Leake D, Boese Q, Scaringe S, Marshall WS, Khvorova A. Rational siRNA design for RNA interference. Nat Biotechnol. 2004 Mar;22(3):326-30. Epub 2004 Feb 01.

Rieder, C. L. and Salmon, E. D. (1998). The vertebrate cell kinetochore and its roles during mitosis. Trends Cell Biol 8, 310-8. 
Rieder, C. L., Cole, R. W., Khodjakov, A. and Sluder, G. (1995). The checkpoint delaying anaphase in response to chormosome monoorientation is mediated by an inhibitory signal produced by unattached kinetochores. Journal of Cell Biology 130, 941-948.

Röber, R. A., Weber, K. and Osborn, M. (1989). Differential timing of nuclear lamin $\mathrm{A} / \mathrm{C}$ expression in the various organs of the mouse embryo and the young animal: a developmental study. Development 105, 365-378.

Röber, R.A., H. Sauter, K. Weber, and M. Osborn. 1990. Cells of the cellular immune and hemopoietic system of the mouse lack lamins $\mathrm{A} / \mathrm{C}$ : distinction versus other somatic cells. J. Cell Sci. 95:587-598.

Rogers, S., Wells, R. and Rechsteiner, M. (1986). Amino acid sequences common to rapidly degraded proteins: the PEST hypothesis. Science 234, 364-368.

Roghi C, Giet R, Uzbekov R, Morin N, Chartrain I, Le Guellec R, Couturier A, Doree M, Philippe M, Prigent C. The Xenopus protein kinase $\mathrm{pEg} 2$ associates with the centrosome in a cell cycle-dependent manner, binds to the spindle microtubules and is involved in bipolar mitotic spindle assembly. J Cell Sci. 1998 Mar;111 ( Pt 5):557-72.

Rowe TM,Sen GC. Organizations and promoter analyses of the human and the mouse genes for PACT, the protein-activator of the interferon-induced protein kinase, PKR. Gene. 2001 Aug 8;273(2):215-25.

Saxton, W. M., Stemple, D. L., Leslie, R. J., Salmon, E. D., Zavortink, M. and McIntosh, J. R. (1984). Tubulin dynamics in cultured mammalian cells. J Cell Biol 99, 2175-86.

Schluter, C., M. Duchrow, C. Wohlenberg, M.H. Becker, G. Key, H.D. Flad, and J. Gerdes. 1993. The cell proliferation-associated antigen of antibody Ki-67: a very large, ubiquitous nuclear protein with numerous repeated elements, representing a new kind of cell cycle-maintaining proteins. J. Cell Biol. 123:513-522.

Schnabel, J. (1998), Über die Wechselwirkungen zwischen Zytokeratinen und die Identifizierung eines neuen humanen Coiled-Coil-Proteins (PhD thesis)

Schwarz DS, Hutvagner G, Du T, Xu Z, Aronin N, Zamore PD. Asymmetry in the assembly of the RNAi enzyme complex. Cell. 2003 Oct 17;115(2):199-208.

Shackleton S, Lloyd DJ, Jackson SN, Evans R, Niermeijer MF, Singh BM, Schmidt H, Brabant G, Kumar S, Durrington PN, Gregory S, O'Rahilly S, Trembath RC. LMNA, encoding lamin A/C, is mutated in partial lipodystrophy. Nat Genet. 2000 Feb; 24(2):153-6.

Shao X, Tarnasky HA, Lee JP, Oko R, van der Hoorn FA. Spag4, a novel sperm protein, binds outer dense-fiber protein Odf1 and localizes to microtubules of manchette and axoneme. Dev Biol. 1999 Jul 1;211(1):109-23. 
Shao, X., Xue, J. and van der Hoorn, F. A. (2001). Testicular protein Spag5 has similarity to mitotic spindle protein Deepest and binds outer dense fiber protein Odf1. Mol Reprod Dev 59, 410-6.

Sharp PA, Zamore PD. Molecular biology. RNA interference. Science. 2000 Mar 31;287(5462):2431-3

Sinensky, M., Fantle, K., Trujillo, M., McLain, T., Kupfer, A. and Dalton, M. (1994). The processing pathway of prelamin A. J Cell Sci 107, 61-67.

Song JJ, Liu J, Tolia NH, Schneiderman J, Smith SK, Martienssen RA, Hannon GJ, Joshua-Tor L. The crystal structure of the Argonaute2 PAZ domain reveals an RNA binding motif in RNAi effector complexes. Nat Struct Biol. 2003 Dec;10(12):102632. Epub 2003 Nov 16.

Soule HD, Vazguez J, Long A, Albert S, Brennan M. A human cell line from a pleural effusion derived from a breast carcinoma. J Natl Cancer Inst. 1973 Nov;51(5):1409-16.

Soutschek J, Akinc A, Bramlage B, Charisse K, Constien R, Donoghue M, Elbashir S, Geick A, Hadwiger P, Harborth J, John M, Kesavan V, Lavine G, Pandey RK, Racie T, Rajeev KG, Rohl I, Toudjarska I, Wang G, Wuschko S, Bumcrot D, Koteliansky V,Limmer Shanoharan W,ornlocher HP. Therapeutic silencing of an endogenous gene by systemic administration of modified siRNAs. Nature. 2004 Nov 11;432(7014):173-8.

Stark, G.R., Kerr, I.M., Williams, B.R., Silverman, R.H. \& Schreiber, R.D. How cells respond to interferons. Annu. Rev. Biochem. 67, 227-264 (1998).

Steinman, R.M., J.M. Silver, and Z.A. Cohn. 1974. Pinocytosis in fibroblasts. J. Cell Biol. 63:949-969.

Sullivan, T., Escalante-Alcalde, D., Bhatt, H., Anver, M., Bhat, N., Nagashima, K., Stewart, C. L. and Burke, B. (1999). Loss of A-type lamin expression compromises nuclear envelope integrity leading to muscular dystrophy. J Cell Biol 147, 913-920.

Sun, G.H., Hirata, Y., Anraku, Y., (1992). Mutations in yeast calmodulin cause defects in spindle pole body functions and nuclear integrity. J. Cell Biol. 119: 16251639

Svoboda, P., Stein, P., Hayashi, H. \& Schultz, R.M. Selective reduction of dormant maternal mRNAs in mouse oocytes by RNA interference. Development 127, 41474156 (2000).

Tabara H, Sarkissian M, Kelly WG, Fleenor J, Grishok A, Timmons L, Fire A, Mello CC. , The rde-1 gene, RNA interference, and transposon silencing in C. elegans. Cell. 1999 Oct 15;99(2):123-32. 
Tam, A., Nouvet, F. J., Fujimura-Kamada, K., Slunt, H., Sisodia, S. S. and Michaelis, S. (1998). Dual roles for Ste24p in yeast a-factor maturation: NH2-terminal proteolysis and $\mathrm{COOH}$-terminal CAAX processing. J Cell Biol 142, 635-649.

Terasaki, M., Song, J., Wong, J. R., Weiss, M. J. and Chen, L. B. (1984). Localization of endoplasmic reticulum in living and glutaraldehyde-fixed cells with fluorescent dyes. Cell 38, 101-108.

Tournebize, R., Popov, A., Kinoshita, K., Ashford, A. J., Rybina, S., Pozniakovsky, A., Mayer, T. U., Walczak, C. E., Karsenti, E. and Hyman, A. A. (2000). Control of microtubule dynamics by the antagonistic activities of XMAP215 and XKCM1 in Xenopus egg extracts. Nat Cell Biol 2, 13-9.

Tuschl T, Zamore PD, Lehmann R, Bartel DP, Sharp PA. Targeted mRNA degradation by double-stranded RNA in vitro. Genes Dev. 1999 Dec 15;13(24):31917.

Tuschl, T. RNA interference and small interfering RNAs. ChemBioChem 2, 239-245

Uchida, E., H. Mizuguchi, A. Ishii-Watabe, and T. Hayakawa. 2002. Comparison of the Efficiency and Safety of Non-viral Vector-Mediated Gene Transfer into a Wide Range of Human Cells. Biol. Pharm. Bull. 25:891-897.

Vasquez, R. J., Gard, D. L. and Cassimeris, L. (1999). Phosphorylation by CDK1 regulates XMAP215 function in vitro. Cell Motil Cytoskeleton 43, 310-21.

Walczak CE, Mitchison TJ, Desai A. XKCM1: a Xenopus kinesin-related protein that regulates microtubule dynamics during mitotic spindle assembly. Cell. 1996 Jan 12;84(1):37-47.

Walczak CE. Microtubule dynamics and tubulin interacting proteins. Curr Opin Cell Biol. 2000 Feb;12(1):52-6. Review.

Walczak, C. E., Vernos, I., Mitchison, T. J., Karsenti, E. and Heald, R. (1998). A model for the proposed roles of different microtubule-based motor proteins in establishing spindle bipolarity. Curr Biol 8, 903-13.

Wang, J., Shen, L., Najafi, H., Kolberg, J., Matschinsky, F. M., Urdea, M. and German, M. (1997). Regulation of insulin preRNA splicing by glucose. Proc Natl Acad Sci U S A 94, 4360-4365.

Waterhouse PM, Wang MB, Finnegan EJ. Role of short RNAs in gene silencing. Trends Plant Sci. 2001 Jul;6(7):297-301

Waterhouse, P.M., Wang, M.B. and Lough, T. (2001) Gene silencing as an adaptive defence against viruses. Nature, 411, 834-842.

Waters JC, Chen RH, Murray AW, Gorbsky GJ, Salmon ED, Nicklas RB. Mad2 binding by phosphorylated kinetochores links error detection and checkpoint action in mitosis.Curr Biol. 1999 Jun 17;9(12):649-652 
Weber K, Pringle JR, Osborn M.Measurement of molecular weights by electrophoresis on SDS-acrylamide gel. Methods Enzymol. 1972;26 PtC:3-27

Weber, K., Plessmann, U. and Traub, P. (1989). Maturation of nuclear lamin A involves a specific carboxy-terminal trimming, which removes the polyisoprenylation site from the precursor; implications for the structure of the nuclear lamina. FEBS Lett 257, 411-414.

Weil, D., L. Garçon, M. Harper, D. Duménil, F. Dautry, and M. Kress. 2002. Targeting the Kinesin Eg5 to Monitor siRNA Transfection in Mammalian Cells. BioTechniques 33:1244-1248.

Wianny, F. and Zernicka-Goetz, M. (2000) Specific interference with gene function by double-stranded RNA in early mouse development. Nat. Cell Biol., 2, 70-75.

Wienholds E, Koudijs MJ, van Eeden FJ, Cuppen E, Plasterk RH. The microRNAproducing enzyme Dicer1 is essential for zebrafish development. Nat Genet. 2003 Nov;35(3):217-8. Epub 2003 Oct 05.

Williams BR. Role of the double-stranded RNA-activated protein kinase (PKR) in cell regulation. Biochem Soc Trans. 1997 May;25(2):509-13. Review.

Winey M. Keeping the centrosome cycle on track. Genome stability. Curr Biol. 1996 Aug 1;6(8):962-4. Review

Wittmann, T., Hyman, A. and Desai, A. (2001). The spindle: a dynamic assembly of microtubules and motors. Nat Cell Biol 3, E28-34.

Worman HJ, Courvalin JC. The nuclear lamina and inherited disease. Trends Cell Biol. 2002 Dec;12(12):591-8. Review.

Xue, J., Tarnasky, H. A., Rancourt, D. E. and van Der Hoorn, F. A. (2002). Targeted disruption of the testicular SPAG5/deepest protein does not affect spermatogenesis or fertility. Mol Cell Biol 22, 1993-7.

Yao, X., Abrieu, A., Zheng, Y., Sullivan, K. F. and Cleveland, D. W. (2000). CENP-E forms a link between attachment of spindle microtubules to kinetochores and the mitotic checkpoint. Nat Cell Biol 2, 484-91.

Yeh E, Skibbens RV, Cheng JW, Salmon ED, Bloom K. Spindle dynamics and cell cycle regulation of dynein in the budding yeast, Saccharomyces cerevisiae.

Yen, T. J., Li, G., Schaar, B. T., Szilak, I. and Cleveland, D. W. (1992). CENP-E is a putative kinetochore motor that accumulates just before mitosis. Nature 359, 536-539.

Yu, H., Chen, Y. K., Feng, S., Dalgarno, D. C., Salmon, E. D. and Bloom, K. (1994). Structural basis for the binding of proline-rich peptides to SH3 domains. Cell 76, 933945. 
Zamore PD. RNA interference: listening to the sound of silence. Nat Struct Biol. 2001 Sep;8(9):746-50. Review

Zamore, P.D., Tuschl, T., Sharp, P.A. \& Bartel, D.P. RNAi: Double-stranded RNA directs the ATP-dependent cleavage of mRNA at 21 to 23 nucleotide intervals. Cell 101, 25-33 (2000).

Zhao Z, Cao Y, Li M, Meng A. Double-stranded RNA injection produces nonspecific defects in zebrafish. Dev Biol. 2001 Jan 1;229(1):215-23. 


\section{SUMMARY}

Gene expression can be silenced in a sequence-specific manner by use of small interfering RNAs (siRNAs) in a process that is known as RNA interference (RNAi). The application of RNAi technologies has the potential to allow the systematic analysis of gene expression and gene function. In this thesis I report novel RNAiinduced phenotypes in mammalian cultured cells for a variety of target genes, and describe some technical aspects of siRNA design, siRNA delivery by a novel method and the application of RNAi in the zebrafish Danio rerio.

The application of RNAi as a research tool to unravel gene function included as target genes the endoprotease FACE1, which is indirectly linked to the Hutchinson Gilford Progeria Syndrome, the coiled coil protein astrin, kinetochore associated proteins (scc1, smc1, smc3, securin and separase) and kinesin related motor proteins (MCAK, TPX2, HSET, KIAA0622). The zinc-finger metalloprotease FACE1 processes the nuclear protein prelamin A to mature lamin A by cleaving off the 16 carboxy terminal amino acid residues. Inhibition of this proteolytic cleavage by silencing of FACE1 results in the accumulation of unprocessed prelamin A and a strong phenotype that includes abnormalities in the nuclear architecture, mitotic arrest and apoptosis. Similar effects are known from HGPS patients in which the FACE1 cleavage site is lost by a cryptic splice site on the lamin A sequence. Using RNAi approaches astrin as well as a set of other mitosis related proteins are shown to be essential for progression through mitosis.

Furthermore silencing with imperfect RNA duplexes is demonstrated. These duplexes consist either of a single, self complementary antisense RNA molecule against the lamin A/C gene (palindrome) or the duplex is composed of two 
complementary antisense RNAs with target regions in the lamin $\mathrm{A} / \mathrm{C}$ sequence (two target site). The most striking experiments show silencing of two target genes by transfection of an imperfect RNA duplex consisting of two antisense RNAs against different targets (dual targeting of lamin $\mathrm{A} / \mathrm{C}$ and emerin).

The successful silencing of a gene depends essentially on the delivery of siRNAs into the target cells. Here I report a novel technique for the transfection of siRNAs by pinocytosis. Cells automatically start pinocytosis when they are confronted with a hypertonic environment. For transfection siRNAs are mixed with an hypertonic medium and reach the cytoplasm of cells in pinocytotic vesicles. Application of an osmotic shock in a hypotonic environment releases the siRNAs which are now accessible for RNAi.

RNAi has been shown for a variety of vertebrate species, including mouse, man and chicken. Conflicting results for the zebrafish indicate incompatibility of zebrafish embryos and siRNA. Delivery of dsRNAs into embryos causes non specific effects. In this thesis I show, that application of siRNA in cell lines from adult and embryonic zebrafish leads to sequence specific gene knockdown without nonspecific effects. 
VIII. Appendix 


\section{VII.2 Tables and Figures}

\section{$\underline{\text { Tables }}$}

Table 1: siRNA sequences and targets (pp. 36-37)

Table 2: Dilutions and sources of antibodies (pp. 46-47)

Table 3: Selected targets for RNA interference and a survey of the effects after silencing (p. 58)

Table 4: Sequences and structures of palindromes. (p. 78)

Table 5: RNAi in zebrafish; cell lines, targets, siRNAs (p. 103)

Table 6: The efficiency of siRNAs targeting human astrin may depend on the $5^{\prime}$ end stability of the antisense strand. (p.121)

\section{Figures}

Figure MI: The principle of pinocytosis mediated transfection. (p. 42)

Figure 1: The pathway of RNAi. (p. 4)

Figure 2: Lamin A mutations related to human diseases. (p. 8)

Figure 3: Anatomy of a synthetic $21 \mathrm{nt}$ siRNA with the essential structural requirements for activity. (p. 14)

Figure 4: Silencing of human FACE1 results in accumulation of prelamin A (p. 53)

Figure 5: Silencing of FACE1 causes abnormalities in nuclear morphology (p.55)

Figure 6: Aberrant mitosis and apoptosis are consequences of FACE1 knockdown. (p. 56)

Figure 7: Double RNAi suggests prelamin A is responsible for phenotypic changes after FACE1 silencing. (p. 58)

Figure 8: Growth rate determination of HL60 cells after transfection of different siRNAs. p. 59

Figure 9: Immunofluorescence detection of FACE1 in the endoplasmic reticulum and the nuclear periphery p. 61

Figure 10: siRNA induced knockdown of astrin in HeLa cells. p. 65

Fig. 11: Astrin silencing induces apoptosis. p. 66 
Figure 12: Graphs visualising data from immunofluorescence and apoptosis detection experiments with different astrin siRNA duplexes. p. 67

Figure 13: Growth rates of cells after transfection of siRNAs against kinetochore related proteins p. 70

Figure 14: Immunofluorescence micrographs afetr RNAi against kinetochore proteins. p. 71

Figure 15: Cell growth after transfection of siRNAs against mitotis related proteins (p. 72)

Figure 16: Cells displayed impaired growth after silencing of kinesin related motor proteins (p. 73)

Figure 17: RNAi against the microtubule destabilizing proteins stathmin and katanin (p. 74)

Figure 18: Strategies for rational siRNA design (p. 77)

Figure 19: Indirect immunofluorescence micrographs of HeLa cells after transfection of s/as and as/as siRNAs. (p. 79)

Figure 20: Western blot analysis of HeLa cells after transfection with palindromic siRNAs (p. 80)

Figure 21: Quantitative western blot analysis of HeLa cell extracts after transfection of s/as and as/as siRNAs. (p. 81)

Figure 22: SiRNA with two target sites leads to knockdown of lamin $\mathrm{A} / \mathrm{C}$ in $\mathrm{HeLa}$ cells. (p. 83)

Figure 23: Quantitative western blot analysis. (p. 84)

Figure 24: Silencing of two targets with a single siRNA (p. 86)

Figure 25: Dual targeting siRNAs in quantitative analysis (p. 88)

Figure 26: SiRNA rescue by destabilisation increases silencing efficiency (p. 89)

Figure 27: Direct demonstration of (A) Uptake of fluorescein-labelled siRNAs by pinocytosis (p. 92)

Figure 28: Pinocytosis mediated silencing of HeLa cells with siRNAs specific for the human kinesin motor protein Eg5. (p. 93)

Figure 29: The silencing efficiency can be increased by using a double pinocytotic and lysis treatment. (p. 95) 
Figure 30: Uptake of siRNAs by the pinocytosis method as a function of concentration and time (p.97)

Figure 31: Comparison of time course of recovery of expression of lamin $\mathrm{A} / \mathrm{C}$ after silencing by the pinocytotic and the cationic lipid methods (p. 98)

Figure 32: Gene silencing of lamin $\mathrm{A} / \mathrm{C}$ in cells of murine origin using the double pinocytosis method (p. 99)

Figure 33: Gene silencing of Ki67 in human HeLa and HEK293 cells using the double pinocytosis method (p. 100)

Figure 34: Silencing of exogenous GFP in zebrafish cells. (p. 104)

Figure 35: ZFL cells show morphological changes after transfection of $\square$-actin siRNA (p. 105)

Figure 36: RNAi in cultured zebrafish cells after transfection of lamin A siRNA (p. 105)

Figure 37: Microinjection of lamin A siRNA into ZFL cells resulted in knockdown of lamin A. (p. 106)

Figure 38: Silencing of lamin B2 in zebrafish cells of adult and embryonic origin (p. 107)

Figure 37: Scheme of micro RNA and RNAi pathways and the asymmetric assembly of RISC (p. 120) 


\section{Statement on the originality of the data}

I collected the data that were presented in this thesis with the exception of the items lisetd below. Parts of the results (text and figures) have been published in my Master's thesis and in journal papers on which I am first author. This far two full publications and two poster abstracts have been published, and a patent has been applied for. A further publication is in pree in the Journal of Cell Science. Two manuscripts are in preparation. A publication list is given on the next page.

Results obtained in collaboration are:

Section: RNAi against the endoprotease FACE1

This study was done in collaboration with Tina Lampe, who was involved in performing experiments and produced figure 5 and parts of figure 6 .

\section{Section: Anatomy of siRNAs}

Most experiments were performed together with Markus Hossbach (MPI for biophysical Chemistry, Göttingen) who also did the analysis of the lamin A/C and emerin sequences to identify of complementary regions. In addition Markus essentially did the siRNA modification used in the rescue experiments.

Section: Pinocytosis mediated transfection of siRNA

Dr. Guido Böse (MPI for biophysical Chemistry, Göttingen) was involved in generating Figure 27. He did the LSM imaging used in the uptake experiments.

Section: RNAi in zebrafish

Silencing of GFP and lamin B2 was done in close experimental collaboration with Heiko Manninga. Materials for GFP silencing (plasmid, siRNAs) were provided by Dr. Erez Raz. 


\section{Publications}

\section{Paper}

Gruber J, Boese G, Tuschl T, Osborn M, Weber K.

RNA interference by osmotic lysis of pinosomes: liposome-independent transfection of siRnas into mammalian cells.

Biotechniques. 2004 Jul;37(1):96-102.

Gruber J, Harborth J, Schnabel J, Weber K, Hatzfeld M.

The mitotic-spindle-associated protein astrin is essential for progression through mitosis.

J Cell Sci. 2002 Nov 1;115(Pt 21):4053-9.

Gruber J, Lampe T, Osborn M and Weber K

RNAi of FACE1 protease results in growth inhibition of human cells expressing lamin A; implications for Hutchinson-Gilford Progeria Syndrome

$\mathrm{J}$ Cell Science (in press)

\section{Master's Thesis}

Jens Gruber

Functional gene analysis in cultured mammalian cells using siRNA mediated gene silencing shows that the human coiled-coil protein astrin is essential for mitosis and cell survival (International Max-Planck Research School Molecular Biology, 2002)

\section{Patent}

Process for transfecting a biologically active nucleic acid into viable cells using pinocytosis

Gruber J, Boese G, Tuschl T, Osborn M, Weber K

\section{Poster abstracts}

The mitotic-spindle-associated protein astrin is essential for progression through mitosis.

Gruber J, Harborth J, Schnabel J, Weber K, Hatzfeld M.

(iLab workshop "Entering an unexplored world. RNA targeting", Wiesbaden, January, 2003, Book of abstracts)

RNA interference by osmotic lysis of pinosomes: liposome-independent transfection of siRnas into mammalian cells

Gruber J, Boese G, Tuschl T, Osborn M, Weber K

(International PhD Sympposium "Horizons", Göttingen, December 2003, Book of abstracts) 


\section{Curriculum Vitae}

\begin{tabular}{ll}
\hline Name & Jens Gruber \\
\hline born in & Northeim \\
date of birth & August 23 ${ }^{\text {rd }}$, 1976
\end{tabular}

\section{Education}

Gerhart-Hauptmann Schule

$1986-1988$

Northeim

Gymnasium Corvinianum

September 1988 -

Northeim

July 1997

(Abitur)

Georg August University

October 1997 - present

Göttingen

(Vordiplom July 1999)

International Max Planck Research

October 2000 - present

School

Göttingen

Max-Planck-Institute for biophysical

September 2001 - present

Chemistry, Department for

Biochemistry and Cell Biology,

Göttingen

M.Sc. Molecular Biology

August 2002

submission of $\mathrm{PhD}$ Thesis

December 2004 